\title{
On the Regularized Fermionic Projector of the Vacuum
}

\author{
Felix Finster
}

January 2008

\begin{abstract}
We construct families of fermionic projectors with spherically symmetric regularization, which satisfy the condition of a distributional $\mathcal{M} P$-product. The method is to analyze regularization tails with a power-law or logarithmic scaling in composite expressions in the fermionic projector. The resulting regularizations break the Lorentz symmetry and give rise to a multilayer structure of the fermionic projector near the light cone. Furthermore, we construct regularizations which go beyond the distributional $\mathcal{M P}$-product in that they yield additional distributional contributions supported at the origin. The remaining freedom for the regularization parameters and the consequences for the normalization of the fermionic states are discussed.
\end{abstract}

\section{Contents}

1 Introduction

2 Preliminaries, Statement of the Main Results 3

2.1 A Variational Principle in Discrete Space-Time . . . . . . . . . . . . . . . . . 3

2.2 Connection to Minkowski Space, the Distributional $\mathcal{M}$.P-Product . . . . . . . . . 6

2.3 Statement of the Main Results . . . . . . . . . . . . . . . . . . . 11

3 The Singularities of $\tilde{\mathcal{M}}$ in Polar Coordinates 12

4 The Momentum Cone Conditions 16

5 The Spherically Symmetric Regularization 22

6 The Outer Strip 29

7 The Intermediate Layers 40

8 The Innermost Layer 46

9 The Regularization Tails near the Origin 52

10 The Regularization Tails near Infinity 54

11 The Continuum Limits of $\mathcal{M}$ and $\mathcal{M} \cdot P$

12 Going Beyond the Distributional $\mathcal{M}$.P-Product 59

13 General Remarks 61

A The Weight Factors $\rho_{\beta}$ 


\section{Introduction}

It is generally believed that the concept of a space-time continuum (such as Minkowski space or a Lorentzian manifold) should be modified for distances as small as the Planck length. Naively, general relativity and quantum field theory become inconsistent on the Planck scale because the energy fluctuations would lead to the formation of microscopic black holes. This led to the intuitive picture that space-time should have a structure with a complicated topology and geometry [17], sometimes referred to as a "quantum foam" [12]. However, this intuitive picture has not yet been made rigorous, and indeed there is not even a consensus on what the correct mathematical framework for physics on the Planck scale is. The most prominent approaches are string theory [13] and loop quantum gravity [18]. Another framework is non-commutative geometry [4], where one works instead of the commutative algebra of functions on a manifold with a non-commutative algebra and replaces the classical action by the so-called spectral action [3]. The requirement of background independence led to the idea that space-time on the Planck scale should be in some sense discrete (see for example [16]). The most notable discrete approaches are the causal sets [2], discrete gauge theory [14, spin networks and spin foam models [1], as well as group field theories describing a simplicial geometry [15].

As an alternative to the above approaches, the principle of the fermionic projector [6] proposes a mathematical framework for Planck scale physics where the physical equations are formulated via a variational principle [6, which is set up in a so-called discrete spacetime for a collection of projectors in an indefinite inner product space (for an introduction to the discrete setting see Section 2.1 below). In the so-called continuum limit [6, Chapter 4], this variational principle can be analyzed in Minkowski space, and one can relate the resulting Euler-Lagrange equations to differential equations for gauge and Dirac fields. The analysis of the continuum limit is based on the assumption that the vacuum corresponds to a Dirac sea configuration, in the sense that the continuum limit of the fermionic projector of the vacuum should be composed of free Dirac seas (see also Section 2.2 below). This assumption clearly needs justification, and it is therefore an important task to show that Dirac sea configurations really are stable minima of our variational principle. In [6, $\S 5.6]$ it is shown that, as a special feature of our particular variational principle, all the composite expressions in the fermionic projector which appear in the stability analysis can be defined as distributions. However, this raises the subtle question of whether there really are regularizations of the fermionic projector with the property that the corresponding composite expressions are so "well behaved" that they converge in the continuum limit to the distributions in $[6, \S 5.6]$. The goal of the present paper is to give the definitive answer "yes" by constructing a family of spherically symmetric regularizations with the desired properties.

Apart from justifying the considerations in [6, §5.6], our analysis also settles a few other important issues. First of all, we will specify the light-cone singularity of $\tilde{\mathcal{M}}$, a distribution which in [6, §5.6] was defined only modulo singular contributions on the light cone (see Section (3). This result is the basis of a stability analysis in the continuum as carried out in [11. Furthermore, we shall see that the analysis here will give no constraints for the socalled regularization parameters as introduced in [6. Chapter 4] (see Remark 13.2). This is important because any additional relations between the regularization parameters would have a sensitive effect on the analysis of the continuum limit. Finally, our analysis will give very strong conditions for the regularization. It seems miraculous that these conditions can all be fulfilled; this can be regarded as a further confirmation for our variational 
principle and the concept of the continuum limit. Despite the fact that we consider only the restrictive class of spherically symmetric regularizations, our analysis seems to reveal a few general properties of admissible regularizations, most notably a multilayer structure near the light cone (see Section 13). If one believes that the regularized fermionic projector describes nature, we thus get concrete hints on how the vacuum should look like on the Planck scale.

Unfortunately, some of the calculations presented in this paper are lengthy and quite tedious. In order to make the paper as convenient to read as possible, we always explain the ideas of the calculations in a non-technical way before entering the details. The calculations in Sections 612 were carried out with the help of a computer algebra program; the corresponding Mathematica worksheets are available from the author on request.

\section{Preliminaries, Statement of the Main Results}

In this section we give a basic introduction to our variational principle in the discrete setting and explain the connection between discrete space-time and Minkowski space. We then formulate our main results.

\subsection{A Variational Principle in Discrete Space-Time}

We let $(H,<. \mid .>)$ be a finite-dimensional complex inner product space. Thus $<. \mid .>$ is linear in its second and anti-linear in its first argument, and it is symmetric,

$$
\overline{\langle\Psi| \Phi>}=<\Phi \mid \Psi>\quad \text { for all } \Psi, \Phi \in H,
$$

and non-degenerate,

$$
<\Psi \mid \Phi>=0 \text { for all } \Phi \in H \quad \Longrightarrow \quad \Psi=0 .
$$

In contrast to a scalar product, <.|.> need not be positive.

A projector $A$ in $H$ is defined just as in Hilbert spaces as a linear operator which is idempotent and self-adjoint,

$$
A^{2}=A \quad \text { and } \quad<A \Psi|\Phi>=<\Psi| A \Phi>\text { for all } \Psi, \Phi \in H .
$$

Let $M$ be a finite set. To every point $x \in M$ we associate a projector $E_{x}$. We assume that these projectors are orthogonal and complete in the sense that

$$
E_{x} E_{y}=\delta_{x y} E_{x} \quad \text { and } \quad \sum_{x \in M} E_{x}=\mathbb{1} .
$$

Furthermore, we assume that the images $E_{x}(H) \subset H$ of these projectors are non-degenerate subspaces of $H$, which all have the same signature $(n, n)$. The parameter $n$ is referred to as the spin dimension. The points $x \in M$ are called discrete space-time points, and the corresponding projectors $E_{x}$ are the space-time projectors. The structure $\left(H,<. \mid .>,\left(E_{x}\right)_{x \in M}\right)$ is called discrete space-time.

We next introduce the so-called fermionic projector $P$ as a projector in $H$ whose image $P(H) \subset H$ is negative definite. The vectors in the image of $P$ have the interpretation as the quantum states of the particles of our system. Thus the rank of $P$ gives the number of particles $f:=\operatorname{dim} P(H)$. The name "fermionic projector" is motivated from the correspondence to Minkowski space, where our particles should go over to Dirac particles, being 
fermions (see Section 2.2). We call the obtained system $\left(H,<. \mid .>,\left(E_{x}\right)_{x \in M}, P\right)$ a fermion system in discrete space-time. For a discussion of the underlying physical principles see [8] or [6, Chapter 4]).

In order to introduce an interaction of the fermions, we now set up a variational principle. For any $u \in H$, we refer to the projection $E_{x} u \in E_{x}(H)$ as the localization of $u$ at $x$. We also use the short notation $u(x)=E_{x} u$ and sometimes call $u(x)$ the wave function corresponding to the vector $u$. Furthermore, we introduce the short notation

$$
P(x, y)=E_{x} P E_{y} \quad x, y \in M .
$$

This operator product maps $E_{y}(H) \subset H$ to $E_{x}(H)$, and it is often useful to regard it as a mapping only between these subspaces,

$$
P(x, y): E_{y}(H) \rightarrow E_{x}(H) .
$$

Using the properties of the space-time projectors (2.1), we find

$$
(P u)(x)=E_{x} P u=\sum_{y \in M} E_{x} P E_{y} u=\sum_{y \in M}\left(E_{x} P E_{y}\right)\left(E_{y} u\right),
$$

and thus

$$
(P u)(x)=\sum_{y \in M} P(x, y) u(y) .
$$

This relation resembles the representation of an operator with an integral kernel, and thus we refer to $P(x, y)$ as the discrete kernel of the fermionic projector. Next we introduce the closed chain $A_{x y}$ as the product

$$
A_{x y}:=P(x, y) P(y, x)=E_{x} P E_{y} P E_{x} ;
$$

it maps $E_{x}(H)$ to itself. Let $\lambda_{1}, \ldots, \lambda_{2 n}$ be the roots of the characteristic polynomial of $A_{x y}$, counted with multiplicities. We define the Lagrangian by

$$
\mathcal{L}[A]=\frac{1}{4 n} \sum_{i, j=1}^{2 n}\left(\left|\lambda_{i}\right|-\left|\lambda_{j}\right|\right)^{2}
$$

and form the action by summing over the space-time points,

$$
\mathcal{S}[P]=\sum_{x, y \in M} \mathcal{L}\left[A_{x y}\right]
$$

Our variational principle is to minimize (2.6), keeping the number of particles $f$ as well as discrete space-time fixed. This variational principle was first introduced in [6]. In [7] it is analyzed mathematically in a more general context (it is there referred to as the auxiliary variational principle in the critical case).

We next derive the corresponding Euler-Lagrange equations (for details see [6, $\S 3.5$ and $\S 5.2]$ ). Suppose that $P$ is a critical point of the action (2.6). We consider a variation $P(\tau)$ of projectors with $P(0)=P$. Denoting the gradient of the Lagrangian by $\mathcal{M}$,

$$
\mathcal{M}[A]_{\beta}^{\alpha}:=\frac{\partial \mathcal{L}[A]}{\partial A_{\alpha}^{\beta}}, \quad \text { with } \alpha, \beta \in\{1, \ldots, 2 n\},
$$


we can write the variation of the Lagrangian as a trace on $E_{x}(H)$,

$$
\delta \mathcal{L}\left[A_{x y}\right]=\left.\frac{d}{d \tau} \mathcal{L}\left[A_{x y}(\tau)\right]\right|_{\tau=0}=\operatorname{Tr}\left(E_{x} \mathcal{M}\left[A_{x y}\right] \delta A_{x y}\right) .
$$

Using the Leibniz rule

$$
\delta A_{x y}=\delta P(x, y) P(y, x)+P(x, y) \delta P(y, x)
$$

together with the fact that the trace is cyclic, after summing over the space-time points we find

$$
\sum_{x, y \in M} \delta \mathcal{L}_{\mu}\left[A_{x y}\right]=\sum_{x, y \in M} 4 \operatorname{Tr}\left(E_{x} Q_{\mu}(x, y) \delta P(y, x)\right)
$$

where we set

$$
Q(x, y)=\frac{1}{4}\left(\mathcal{M}\left[A_{x y}\right] P(x, y)+P(x, y) \mathcal{M}\left[A_{y x}\right]\right) .
$$

It follows from general properties of the spectral decompositions of $A_{x y}$ and $A_{y x}$ (see [6, Lemma 5.2.1]) that the two summands on the right coincide, and thus we can write $Q(x, y)$ as the product

$$
Q(x, y)=\frac{1}{2} \mathcal{M}\left[A_{x y}\right] P(x, y) .
$$

Thus the first variation of the action can be written in the compact form

$$
\delta \mathcal{S}[P]=4 \operatorname{Tr}(Q \delta P),
$$

where $Q$ is the operator in $H$ with kernel (2.8). This equation can be simplified using that the operators $P(\tau)$ are all projectors of fixed rank. Namely, there is a family of unitary operators $U(\tau)$ with $U(\tau)=\mathbb{1}$ and

$$
P(\tau)=U(\tau) P U(\tau)^{-1}
$$

Hence $\delta P=i[B, P]$, where $B=-i U^{\prime}(0)$ is the infinitesimal generator of the family $U(\tau)$. Using this relation in (2.9) and again using that the trace is cyclic, we find $\delta \mathcal{S}[P]=$ $4 i \operatorname{Tr}([P, Q] B)$. Since $B$ is an arbitrary self-adjoint operator, we conclude that

$$
[P, Q]=0 \text {. }
$$

This commutator equation with $Q$ given by (2.8) are the Euler-Lagrange equations corresponding to our variational principle.

Before moving on to the space-time continuum, we briefly mention a few results on fermion systems in discrete space-time. In [9] it is shown under under general assumptions that the permutation symmetry of the space-time points is spontaneously broken by the fermionic projector. This implies that the fermionic projector induces nontrivial relations between the space-time points. In particular, one can introduce a discrete causal structure [8]. In [5] the spontaneous symmetry breaking and the emergence of a discrete causal structure are illustrated in simple examples. 


\subsection{Connection to Minkowski Space, the Distributional $\mathcal{M}$.P-Product}

It is conjectured that, in a suitable limit where the number of particles and space-time points tends to infinity, the emergent discrete causal structure gives rise to the local and causal structure of Minkowski space. For a discussion of this conjecture we refer to the recent survey article [10]. Here we focus on a particular aspect of this problem, namely to the question of how the Euler-Lagrange equations (2.10) can be introduced for vacuum Dirac sea configurations in Minkowski space. The crucial point is to make sense of the gradient of the Lagrangian (2.7) and of the product of $\mathcal{M}$ with $P$ in the definition of $Q$, (2.8).

Before we can specify what needs to be done, we need to get a connection between discrete space-time and Minkowski space. The simplest method for obtaining a correspondence to relativistic quantum mechanics in Minkowski space is to replace the discrete space-time points $M$ by the space-time continuum $\mathbb{R}^{4}$ and the sums over $M$ by spacetime integrals. For a vector $\Psi \in H$, the corresponding localization $E_{x} \Psi$ should be a four-component Dirac wave function, and the scalar product $\langle\Psi(x)| \Phi(x)>$ on $E_{x}(H)$ should correspond to the usual Lorentz invariant scalar product on Dirac spinors $\bar{\Psi} \Phi$ with $\bar{\Psi}=\Psi^{\dagger} \gamma^{0}$ the adjoint spinor. Since this last scalar product is indefinite of signature $(2,2)$, we are led to choosing $n=2$. In view of (2.3), the discrete kernel should in the continuum go over to the integral kernel of an operator $P$ on the Dirac wave functions,

$$
(P \Psi)(x)=\int P(x, y) \Psi(y) d^{4} y .
$$

The image of $P$ should be spanned by the occupied fermionic states. We take Dirac's concept literally that in the vacuum all negative-energy states are occupied by fermions forming the so-called Dirac sea. Thus we are led to describe the vacuum by the integral over the lower mass shell

$$
P(x, y)=\int \frac{d^{4} k}{(2 \pi)^{4}}(\not k+m) \delta\left(k^{2}-m^{2}\right) \Theta\left(-k^{0}\right) e^{-i k(x-y)}
$$

(here $\Theta$ is the Heaviside function). In order to take into account the three generations of elementary particles (such as the quarks $u, s, t$ in the standard model), we take the sum of three Dirac seas,

$$
P(x, y)=\sum_{\beta=1}^{3} \rho_{\beta} \int \frac{d^{4} k}{(2 \pi)^{4}}\left(\not k+m_{\beta}\right) \delta\left(k^{2}-m_{\beta}^{2}\right) \Theta\left(-k^{0}\right) e^{-i k(x-y)} .
$$

Compared to the situation in [6, $\S 2.2]$, the ansatz (2.11) is more general in that it involves additional weight factors $\rho_{\beta}>0$. We treat these factors as a priori given positive constants. For a discussion of the physical significance and the implications of the weights $\rho_{\beta}$ see Appendix A, More generally, in [6, §5.1] a realistic system of fermions is built up by taking a direct sum of operators acting on so-called sectors. In this more general situation, our variational principle splits into separate variational principles in the individual sectors. Thus we may restrict attention to one sector, and considering a massive sector again gives a fermionic projector of the form (2.11).

The Fourier integrals in (2.11) are clearly well defined in the distributional sense. Carrying them out using Bessel functions (see [6, §2.5] and Section 3 below), one sees that $P(x, y)$ is even a smooth function away from the light cone (i.e. for $(y-x)^{2} \neq 0$ ), but 
that it has poles and singularities on the light cone. Before we can set up our variational principle, these singularities must be removed by a regularization procedure. To us, the regularization has a physical significance in that a suitably regularized fermionic projector should describe the physical fermionic projector in discrete space-time. Since we do not have any information on what the physically correct regularization is, we use the method of variable regularization and consider a sufficiently large class of regularizations. More precisely, as in [6, §4.1] we assume that the regularizations are homogeneous and have a vector-scalar structure. This means that the regularized fermionic projector, which we denote by $P^{\varepsilon}$, can be written as a Fourier integral

$$
P^{\varepsilon}(x, y)=\int \frac{d^{4} k}{(2 \pi)^{4}} \hat{P}^{\varepsilon}(k) e^{-i k(x-y)},
$$

where $\hat{P}^{\varepsilon}$ is a distribution of the form

$$
\hat{P}^{\varepsilon}(k)=\hat{g}_{j}(k) \gamma^{j}+\hat{h}(k)
$$

with real-valued distributions $\hat{g}_{j}$ and $\hat{h}$. Here the parameter $\varepsilon>0$ denotes the length scale of the regularization. Thus, expressed in momentum space, the distributions $\hat{g}_{j}$ and $\hat{h}$ should decay at infinity on the scale $k \sim \varepsilon^{-1}$. Furthermore, we restrict attention to spherically symmetric regularizations which are composed of surface states. The last assumption means that, similar to the situation in (2.11), the distributions $\hat{g}_{j}$ and $\hat{h}$ should be supported on three hypersurfaces (for details see (5.1) and Section 5). We consider a family of regularized fermionic projectors $\left(P^{\varepsilon}\right)_{\varepsilon>0}$ with the above properties. As $\varepsilon$ tends to zero, the regularized fermionic projectors should go over to the unregularized fermionic projector,

$$
\lim _{\varepsilon \searrow 0} P^{\varepsilon}(x, y)=P(x, y) \quad \text { as a distribution. }
$$

Having defined the fermionic projector, we can introduce the closed chain in analogy to (2.4) by

$$
A_{x y}^{\varepsilon}=P^{\varepsilon}(x, y) P^{\varepsilon}(y, x) .
$$

For ease in notation, we will often omit the subscript ' $x y$ '. In our setting of one sector and a vector-scalar structure, the roots of the characteristic polynomials of $A^{\varepsilon}$ have a particularly simple structure.

Lemma 2.1 For the fermionic projector (2.12, 2.13), the characteristic polynomial of the closed chain $A_{x y}^{\varepsilon}$ has two roots $\lambda_{ \pm}$, each of multiplicity two. Either the $\lambda_{ \pm}$form a complex conjugate pair, $\overline{\lambda_{+}}=\lambda_{-}$, or else the $\lambda_{ \pm}$are both real and have the same sign.

Proof. According to (2.12, 2.13) and the fact that the distributions $\hat{g}_{j}$ and $\hat{h}$ are realvalued, we can write the fermionic projector in position space as

$$
P^{\varepsilon}(x, y)=g_{j}(x, y) \gamma^{j}+h(x, y), \quad P^{\varepsilon}(y, x)=\overline{g_{j}(x, y)} \gamma^{j}+\overline{h(x, y)} .
$$

Thus, omitting the arguments $x$ and $y$,

$$
A^{\varepsilon}=(\not g+h)(\bar{g}+\bar{h}) .
$$

A short calculation using the anti-commutation relations of the Dirac matrices shows that the characteristic polynomial of $A^{\varepsilon}$ has the two roots

$$
\lambda_{ \pm}=g \bar{g}+h \bar{h} \pm \sqrt{(g \bar{g})^{2}-g^{2} \bar{g}^{2}+(g \bar{h}+h \bar{g})^{2}}
$$


(for more details see [6, $§ 5.3]$; we use the short notations $g \bar{g} \equiv g_{j} \overline{g^{j}}$ and $g^{2} \equiv g_{j} g^{j}$, $\bar{g}^{2} \equiv \overline{g_{j} g^{j}}$. If the discriminant is negative, the $\lambda_{ \pm}$form a complex conjugate pair. If conversely the discriminant is positive, the $\lambda_{ \pm}$are both real. In order to show that they have the same sign, we compute their product,

$$
\begin{aligned}
\lambda_{+} \lambda_{-} & =(g \bar{g}+h \bar{h})^{2}-\left[(g \bar{g})^{2}-g^{2} \bar{g}^{2}+(g \bar{h}+h \bar{g})^{2}\right] \\
& =2(g \bar{g})|h|^{2}+|h|^{4}+g^{2} \bar{g}^{2}-(g \bar{h}+h \bar{g})^{2} \\
& =|h|^{4}+g^{2} \bar{g}^{2}-g^{2} \bar{h}^{2}-h^{2} \bar{g}^{2} \\
& =\left(g^{2}-h^{2}\right)\left(\bar{g}^{2}-\bar{h}^{2}\right) \geq 0 .
\end{aligned}
$$

Using this lemma, we can simplify the Lagrangian (2.5) to

$$
\mathcal{L}[A]=\left\{\begin{array}{cl}
\left(\lambda_{+}-\lambda_{-}\right)^{2} & \text { if } \lambda_{ \pm} \in \mathbb{R} \\
0 & \text { if } \lambda_{ \pm} \notin \mathbb{R}
\end{array}\right.
$$

Furthermore, we can easily compute $\mathcal{M}$ as defined by (2.7). Namely, in the case $\lambda_{ \pm} \notin$ $\mathbb{R}$, the roots of the characteristic polynomial by continuity will be non-real on an open neighborhood of $A$. However, the degeneracy will in general not be preserved, and thus the spectrum of $A$ will consist of two complex conjugate pairs $\lambda_{1}, \overline{\lambda_{1}}$ and $\lambda_{2}, \overline{\lambda_{2}}$ (possibly with $\lambda_{1}=\lambda_{2}$ ). The Lagrangian (2.5) then becomes

$$
\mathcal{L}[A]=\left(\left|\lambda_{1}\right|-\left|\lambda_{2}\right|\right)^{2} .
$$

According to standard perturbation theory with degeneracies, the spectrum is Lipschitz continuous in $A$. Hence (2.17) is differentiable at the point $\lambda_{1}=\lambda_{2}$, and its gradient vanishes. If conversely $\lambda_{ \pm} \in \mathbb{R}$ and $\lambda_{+} \neq \lambda_{-}$, these properties will again be preserved in a neighborhood of $A$. In this neighborhood, we can write (2.16) as

$$
\mathcal{L}[A]=\operatorname{Tr}\left(A^{2}\right)-\frac{1}{4} \operatorname{Tr}(A)^{2},
$$

and varying this Lagrangian gives two times the trace-free part of $A$,

$$
\mathcal{M}[A]=2 A-\frac{1}{2} \operatorname{Tr}(A) \mathbb{1} .
$$

In the remaining case $\lambda_{+}=\lambda_{-} \in \mathbb{R}$, the Lagrangian (2.16) is continuously differentiable and has vanishing gradient. We thus obtain

$$
\mathcal{M}[A]=\left\{\begin{array}{cl}
2 A-\frac{1}{2} \operatorname{Tr}(A) \mathbb{1} & \text { if } \lambda_{ \pm} \in \mathbb{R} \\
0 & \text { if } \lambda_{ \pm} \notin \mathbb{R} .
\end{array}\right.
$$

In the region away from the light cone, where $P(x, y)$ is a smooth function, the matrix $\mathcal{M}\left[A_{x y}\right]$ is well defined even without regularization. We postpone the detailed calculations to Section 3 , here it suffices to derive the general structure of $\mathcal{M}\left[A_{x y}\right]$ from the following simple consideration. For given space-time points $x$ and $y$ with $(y-x)^{2} \neq 0$, we know from the Lorentz symmetry that the unregularized fermionic projector (2.11) is of the form

$$
P(x, y)=\alpha \not{s}+\beta \mathbb{1}, \quad P(y, x)=\bar{\alpha} \not{\phi}+\bar{\beta} \mathbb{1}
$$


for some complex parameters $\alpha$ and $\beta$, where we set $\xi \equiv y-x$. As a consequence,

$$
A_{x y}=a \not s+b \mathbb{1}=A_{y x}
$$

with real parameters $a=\alpha \bar{\beta}+\beta \bar{\alpha}$ and $b=|\alpha|^{2} \xi^{2}+|\beta|^{2}$. Suppose that the vector $\xi$ is spacelike. Then there is a Lorentz "rotation" which transforms $\xi$ to $-\xi$. Using the Lorentz symmetry of $A_{x y}$, we find that $A_{y x}$ is obtained from $A_{x y}$ simply by the replacement $\xi \rightarrow-\xi$,

$$
A_{y x}=-a \not s+b \mathbb{1} \text {. }
$$

This is consistent with the right equation in (2.19) only if $a$ vanishes. We conclude that $A_{x y}$ is of the form

$$
A_{x y}=\left\{\begin{array}{cl}
a \not{\phi}+b \mathbb{1} & \text { if } \xi \text { is timelike } \\
b \mathbb{1} & \text { if } \xi \text { is spacelike } .
\end{array}\right.
$$

In the case when $\xi$ is timelike, our last argument does not apply because a Lorentz transformation which maps $\xi$ to $-\xi$ involves a reversal of the time direction, but the fermionic projector (2.11) has no time reflection symmetry as it distinguishes the lower from the upper mass shells. However, if $\xi$ is timelike, the identity $\xi^{2}=\xi^{2}>0$ shows that the matrix $\not \mathcal{~ h a s ~ r e a l ~ e i g e n v a l u e s . ~ W e ~ t h u s ~ o b t a i n ~ f r o m ~ ( 2 . 1 8 ) ~ t h a t ~}$

$$
\mathcal{M}\left[A_{x y}\right]=\left\{\begin{array}{cl}
2 a \not z & \text { if } \xi \text { is timelike } \\
0 & \text { if } \xi \text { is spacelike. }
\end{array}\right.
$$

Moreover, it is clear from (2.19) that $\mathcal{M}\left[A_{x y}\right]=\mathcal{M}\left[A_{y x}\right]$ and, again using Lorentz symmetry, we conclude that there is a real function $f$ such that

$$
\mathcal{M}\left[A_{x y}\right]=\left\{\begin{array}{cl}
\not \epsilon\left(\xi^{0}\right) f\left(\xi^{2}\right) & \text { if } \xi \text { is timelike } \\
0 & \text { if } \xi \text { is spacelike }
\end{array}\right.
$$

( $\epsilon$ denotes the step function $\epsilon(x)=1$ if $x>0$ and $\epsilon(x)=-1$ otherwise). It is important to observe that $\mathcal{M}\left[A_{x y}\right]$ vanishes for spacelike $y-x$ and in this way encodes the causal structure of Minkowski space.

Clearly, we cannot use the above argument on the light cone $\xi^{2}=0$, where $\mathcal{M}\left[A_{x y}\right]$ is ill-defined. Indeed, the function $f\left(\xi^{2}\right)$ has a non-integrable pole as $\xi^{2} \searrow 0$ (for details see Section [3), and therefore $\mathcal{M}\left[A_{x y}\right]$ has a serious singularity on the light cone. If we regularized by replacing $P$ in the above construction by $P^{\varepsilon}$, the resulting $\mathcal{M}\left[A_{x y}^{\varepsilon}\right]$ would be well defined for all $\xi$. Qualitatively speaking, the singularity on the light cone would be "smeared out" on the scale $\varepsilon$, whereas away from the light cone $\mathcal{M}\left[A_{x y}^{\varepsilon}\right]$ would be wellapproximated by the unregularized $\mathcal{M}\left[A_{x y}\right]$ (at least for the regularizations considered in this paper, which all satisfy the condition introduced in [6, §5.6] that $P^{\varepsilon}$ should be macroscopic away from the light cone). However, it is far from obvious what happens in the limit $\varepsilon \searrow 0$, and this is precisely the question which we shall address here. We will see that in this limit, $\mathcal{M}\left[A_{x y}\right]$ will in general develop singularities on the light cone, which diverge even in the distributional sense. However, we will show that there are special regularizations where $\mathcal{M}\left[A_{x y}\right]$ does indeed converge as a distribution. Before we can state our result, we must briefly recall the procedure in [6, §5.6]. In order to make sense of the pole of $\mathcal{M}\left[A_{x y}\right]$ across the light cone, a distribution $\tilde{\mathcal{M}}(\xi)$ is introduced which coincides with $\mathcal{M}\left[A_{x y}\right]$ away from the light cone. $\tilde{\mathcal{M}}(\xi)$ can be represented as the distributional derivative of a function $F$ which has an integrable pole on the light cone and can thus be regarded as a regular distribution. More precisely,

$$
\tilde{\mathcal{M}}(\xi)=\not_{\xi} \square_{\xi} F(\xi),
$$




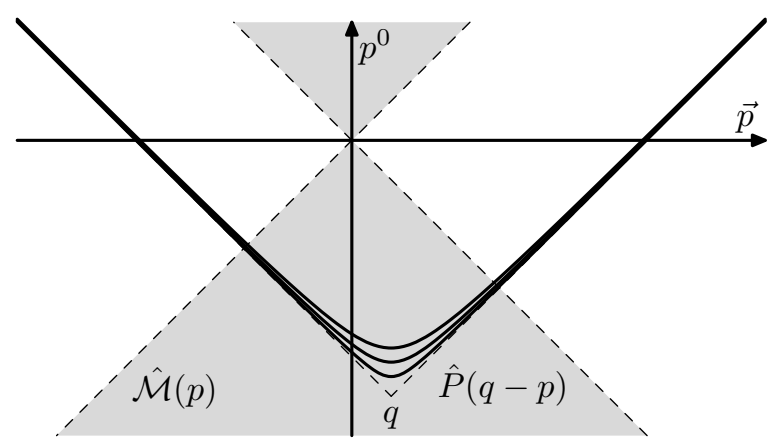

Figure 1: The convolution $\hat{\mathcal{M}} * \hat{P}$.

where $F$ is causal, Lorentz invariant and odd under time reversals, i.e.

$$
F(\xi)=\Theta\left(\xi^{2}\right) \epsilon\left(\xi^{0}\right) g\left(\xi^{2}\right)
$$

for a suitable real function $g\left(\xi^{2}\right)$ which has an integrable pole as $\xi^{2} \searrow 0$. Let us consider the Fourier transform of $\tilde{\mathcal{M}}(\xi)$, which we denote for convenience by $\hat{\mathcal{M}}(k)$ (omitting the tilde cannot lead to confusion because the Fourier transform of $\mathcal{M}\left[A_{x y}\right]$ is ill-defined due to the singularities on the light cone). The differential operators in (2.21) clearly correspond to multiplication operators in momentum space,

$$
\hat{\mathcal{M}}(k)=-i \not k k^{2} \hat{F}(k) .
$$

As in [6], we denote the mass cone by $\mathcal{C}=\left\{k \mid k^{2}>0\right\}$ and define the upper and lower mass cone by $\mathcal{C}^{\vee}=\left\{k \in \mathcal{C} \mid k^{0}>0\right\}$ and $\mathcal{C}^{\wedge}=\left\{k \in \mathcal{C} \mid k^{0}<0\right\}$, respectively. The following argument shows that $\hat{F}$ vanishes identically outside the closed mass cone. Suppose that $k \notin \overline{\mathcal{C}}$. Due to Lorentz symmetry, we can assume that $k$ is purely spatial, $k=(0, \vec{k})$. When we now take the Fourier transform of (2.22), the integrand of the resulting time integral is odd because of the step function $\epsilon\left(\xi^{0}\right)$, and thus the whole integral vanishes. In view of (2.23), we come to the important conclusion that also $\hat{\mathcal{M}}$ is supported inside the mass cone,

$$
\operatorname{supp} \hat{\mathcal{M}} \subset \overline{\mathcal{C}}
$$

This support property makes it possible to make sense of the pointwise product in (2.8) even without regularization. Namely, setting

$$
Q(x, y)=\frac{1}{2} \tilde{\mathcal{M}}(\xi) P(x, y)
$$

we rewrite the product as a convolution in momentum space,

$$
\hat{Q}(q)=\frac{1}{2}(\hat{\mathcal{M}} * \hat{P})(q)=\frac{1}{2} \int \frac{d^{4} p}{(2 \pi)^{4}} \hat{\mathcal{M}}(p) \hat{P}(q-p) .
$$

If $q$ is inside the lower mass cone, the last integrand has compact support (see Figure 1), and the integral is finite. If however $q \notin \overline{\mathcal{C}^{\wedge}}$, the convolution integral extends over an unbounded region and is thus ill-defined.

In [6, §5.6], the regularization of the product in (2.8) is treated using an ad-hoc assumption, which in our setting can be stated as follows. 
Definition 2.2 The regularized fermionic projector $\left(P^{\varepsilon}\right)_{\varepsilon>0}$ satisfies the assumption of a distributional $\mathcal{M} P$-product if the following conditions are satisfied:

(i) There is a distribution $\tilde{\mathcal{M}}(\xi)$ of the form (2.21, 2.22) such that $\lim _{\varepsilon \searrow 0} \mathcal{M}\left[A_{x y}^{\varepsilon}\right]=\tilde{\mathcal{M}}(\xi)$ in the distributional sense.

(ii) For every $k$ for which $\lim _{\varepsilon}{ }_{0} \hat{Q}^{\varepsilon}(k)$ exists, the convolution integral (2.25) is well defined and $\lim _{\varepsilon \searrow 0} \hat{Q}^{\varepsilon}(k)=\hat{Q}(k)$.

\subsection{Statement of the Main Results}

The main result of the present paper is to justify the assumption of a distributional $\mathcal{M P}$ product in the following sense.

Theorem 2.3 Suppose that $\tilde{\mathcal{M}}(\xi)$ is a distribution which away from the light-cone coincides with $\mathcal{M}\left[A_{x y}\right]$, (2.18), and can be represented in the form (2.21, 2.22) with $F$ a regular distribution. Then there is a family of regularized fermionic projectors $\left(P^{\varepsilon}\right)_{\varepsilon>0}$ with the following properties:

(1) The $P^{\varepsilon}$ satisfy (2.14), are homogeneous (2.12) and have vector-scalar structure (2.13). Furthermore, they are spherically symmetric and are composed of surface states.

(2) $\lim _{\varepsilon \searrow 0} \mathcal{M}\left[A_{x y}^{\varepsilon}\right]=\tilde{\mathcal{M}}(\xi)$ in the distributional sense.

(3) For every $k \in \mathcal{C}^{\wedge}$,

$$
\lim _{\varepsilon \searrow 0} \hat{Q}^{\varepsilon}(k)=\hat{Q}(k)
$$

with $\hat{Q}(k)$ as defined by (2.25).

(4) For every $k \notin \overline{\mathcal{C}^{\wedge}}, \hat{Q}^{\varepsilon}(k)$ diverges in the sense that

$$
\lim _{\varepsilon \searrow 0} \inf \sigma\left(\hat{Q}^{\varepsilon}(k)\right)=+\infty .
$$

The constructions used for proving this theorem leave us with the freedom to modify $Q$ by distributional contributions supported at the origin $\xi=0$. Expressed in momentum space, we prove the following generalization of Theorem 2.3 .

Theorem 2.4 Under the assumptions of Theorem [2.3, for any real parameters $c_{2}, c_{3}, c_{4}$ there is a family of regularized fermionic projectors $\left(P^{\varepsilon}\right)_{\varepsilon>0}$, which has the properties (1), (2) and (4) in the statement of Theorem 2.3 and instead of (3) satisfies the condition

(3') For every $k \in \mathcal{C}^{\wedge}$,

$$
\lim _{\varepsilon \searrow 0} \hat{Q}^{\varepsilon}(k)=\frac{1}{2}(\hat{\mathcal{M}} * \hat{P})(k)+c_{2}+c_{3} \not k+c_{4} k^{2} .
$$

The proofs of these theorems are constructive and will give us detailed information on the admissible regularizations. This will be discussed in Section 13 . 


\section{The Singularities of $\tilde{\mathcal{M}}$ in Polar Coordinates}

We begin the explicit calculations by determining the function $f$ in (2.20). Clearly, we may assume that the vector $\xi$ is timelike and future directed. Then the Fourier integral (2.12) can be given explicitly in terms of Bessel functions, which in turn have a series expansion,

$$
\begin{aligned}
P(x, y) & =\sum_{\beta=1}^{3} \rho_{\beta}\left(i \not \partial_{x}+m_{\beta}\right)\left(a_{\beta}\left(\xi^{2}\right)+i b_{\beta}\left(\xi^{2}\right)\right) \\
a_{\beta}\left(\xi^{2}\right) & =\frac{1}{2} \int \frac{d^{4} k}{(2 \pi)^{4}} \delta\left(k^{2}-m_{\beta}^{2}\right) e^{-i k(x-y)}=\frac{m_{\beta}^{2}}{16 \pi^{2}} \frac{Y_{1}\left(m_{\beta} \sqrt{\xi^{2}}\right)}{m_{\beta} \sqrt{\xi^{2}}} \\
& =-\frac{1}{8 \pi^{3} \xi^{2}}+\frac{m_{\beta}^{2}}{32 \pi^{3}}\left(\log \left(m_{\beta}^{2} \xi^{2}\right)+c\right)+\mathcal{O}\left(\xi^{2} \log \xi^{2}\right) \\
b_{\beta}\left(\xi^{2}\right) & =\frac{i}{2} \int \frac{d^{4} k}{(2 \pi)^{4}} \delta\left(k^{2}-m_{\beta}^{2}\right) \epsilon\left(k^{0}\right) e^{-i k(x-y)}=\frac{m_{\beta}^{2}}{16 \pi^{2}} \frac{J_{1}\left(m_{\beta} \sqrt{\xi^{2}}\right)}{m_{\beta} \sqrt{\xi^{2}}} \\
& =\frac{m_{\beta}^{2}}{32 \pi^{2}}-\frac{m_{\beta}^{4}}{256 \pi^{2}} \xi^{2}+\mathcal{O}\left(\xi^{4}\right),
\end{aligned}
$$

where $c=2 C-2 \log 2-1$ and $C$ is Euler's constant. Using that the functions $a_{\beta}$ and $b_{\beta}$ are real, a short calculation yields that

$$
A_{x y}-\frac{1}{4} \operatorname{Tr}\left(A_{x y}\right) \mathbb{1}=-2 \not \sum_{\alpha, \beta=1}^{3}\left(a_{\alpha}^{\prime} m_{\beta} b_{\beta}+m_{\alpha} b_{\alpha} a_{\beta}^{\prime}-b_{\alpha}^{\prime} m_{\beta} a_{\beta}-m_{\alpha} a_{\alpha} b_{\beta}^{\prime}\right),
$$

and comparing with (2.18, 2.20), we obtain the simple formula

$$
f=-8 \sum_{\alpha, \beta=1}^{3}\left(a_{\alpha}^{\prime} m_{\beta} b_{\beta}-m_{\alpha} a_{\alpha} b_{\beta}^{\prime}\right) .
$$

Substituting the above asymptotic expansions of the Bessel functions, we find that $f$ really has a non-integrable pole. More precisely,

$$
f(z)=\frac{\mathfrak{m}_{3}}{z^{2}}+\frac{\mathfrak{m}_{5}}{z}+\mathcal{O}(\log z),
$$

where we set $z \equiv \xi^{2}$ and introduced the abbreviations

$$
\begin{aligned}
\mathfrak{m}_{3} & =-\frac{1}{64 \pi^{5}} \sum_{\alpha, \beta=1}^{g} \rho_{\alpha} \rho_{\beta}\left(m_{\alpha}^{3}+m_{\beta}^{3}\right) \\
\mathfrak{m}_{5} & =\frac{1}{512 \pi^{5}} \sum_{\alpha, \beta=1}^{g} \rho_{\alpha} \rho_{\beta}\left(m_{\alpha}-m_{\beta}\right)^{2}\left(m_{\alpha}+m_{\beta}\right)^{3} .
\end{aligned}
$$

Next we compute the function $g$ in (2.22). The Dirac operator and the Laplacian of a Lorentz invariant function $h\left(\xi^{2}\right)$ are computed by

$$
\not \partial h(z)=2 \not h^{\prime}(z), \quad \square h(z)=\frac{4}{z}\left(z^{2} h^{\prime}(z)\right)^{\prime} .
$$


Using these formulas, it is immediately verified that the function $g(z)$, defined for given real constants $c_{0}, c_{1}$ and any $z>0$ by

$$
g(z)=\frac{1}{8} \int_{1}^{z} \frac{d \tau}{\tau^{2}} \int_{0}^{\tau} \sigma d \sigma \int_{1}^{\sigma} f+\frac{c_{0}}{8}+\frac{c_{1}}{16} z
$$

in the interior of the light cone is a solution of the equation

$$
\not ु f(z)=\not \partial \square g(z) \text {. }
$$

Furthermore, using (3.5) in (3.6) one sees that $g$ has only a logarithmic pole as $z \searrow$ 0 . Therefore, we can use (2.22) to define $F$ as a regular distribution, and taking its distributional derivative (2.21) gives the distribution $\tilde{\mathcal{M}}(\xi)$, which coincides away from the light cone with the function $\mathcal{M}\left[A_{x y}\right]$. The parameters $c_{0}$ and $c_{1}$ can be interpreted as integration constants. From the calculations

$$
\begin{aligned}
\not \partial \square\left(\Theta\left(\xi^{2}\right) \epsilon\left(\xi^{0}\right)\right) & =4 \not \partial\left(\delta\left(\xi^{2}\right) \epsilon\left(\xi^{0}\right)\right)=8 \not \delta^{\prime}\left(\xi^{2}\right) \epsilon\left(\xi^{0}\right) \\
\not \supset \square\left(\xi^{2} \Theta\left(\xi^{2}\right) \epsilon\left(\xi^{0}\right)\right) & =8 \not \partial\left(\Theta\left(\xi^{2}\right) \epsilon\left(\xi^{0}\right)\right)=16 \not z \delta\left(\xi^{2}\right) \epsilon\left(\xi^{0}\right)
\end{aligned}
$$

one sees that they give rise to contributions to $\tilde{\mathcal{M}}$ supported on the light cone,

$$
\frac{\partial}{\partial c_{0}} \tilde{\mathcal{M}}(x, y)=\not \delta^{\prime}\left(\xi^{2}\right) \epsilon\left(\xi^{0}\right), \quad \frac{\partial}{\partial c_{1}} \tilde{\mathcal{M}}(x, y)=\not \delta\left(\xi^{2}\right) \epsilon\left(\xi^{0}\right)
$$

Our next lemma shows that the distribution $\tilde{\mathcal{M}}(\xi)$ is regular at the origin $\xi=0$. We let $\eta \in C_{0}^{\infty}\left(\mathbb{R}^{4}\right)$ be a test function which is identically equal to one in a neighborhood of the origin. We set for any $\delta>0$

$$
\eta_{\delta}(\xi)=\eta\left(\frac{\xi}{\delta}\right)
$$

Lemma $3.1 \lim _{\delta \searrow 0} \eta_{\delta}(\xi) \tilde{\mathcal{M}}(\xi)=0$ in the distributional sense.

Proof. According to (2.21), we have for any test function $h \in C_{0}^{\infty}\left(\mathbb{R}^{4}\right)$,

$$
\begin{aligned}
\int \eta_{\delta}(\xi) \tilde{\mathcal{M}}(\xi) h(\xi) d^{4} \xi & =-\int F(\xi) \square_{\xi} \not_{\xi}\left(h(\xi) \eta_{\delta}(\xi)\right) d^{4} \xi \\
& =-\delta \int_{\operatorname{supp} \eta} F(\delta \zeta) \square_{\zeta} \not_{\zeta}(h(\delta \zeta) \eta(\zeta)) d^{4} \zeta
\end{aligned}
$$

where in the last step we introduced the new variable $\zeta=\xi / \delta$. Estimating the integrand on the support of $\eta$ by

$$
|F(\delta \zeta)| \leq c|F(\zeta)| \log \delta, \quad\left|\square_{\zeta} \not \phi_{\zeta}(h(\delta \zeta) \eta(\zeta))\right| \leq c,
$$

we can bound the integral by a constant times $\log \delta$.

It remains to analyze the singularities of $\tilde{\mathcal{M}}(\xi)$ on the light cone, away from the origin. Due to the symmetry under the transformation $\xi \rightarrow-\xi$, we may restrict attention to the future light cone. Thus choosing polar coordinates $\left(t=\xi^{0}, r=|\vec{\xi}|, \vartheta, \varphi\right)$, we consider the region $t \approx r>0$, and thus it is convenient to introduce the "small" time variable $s=t-r$. 
Furthermore, due to spherical symmetry, we can decompose $\tilde{\mathcal{M}}$ into a time and a radial component as follows,

$$
\tilde{\mathcal{M}}=\tilde{\mathcal{M}}^{0} \gamma^{0}-\tilde{\mathcal{M}}^{r} \gamma^{r}
$$

where we set

$$
\gamma^{r}=\frac{\vec{\xi} \vec{\gamma}}{r}
$$

and $\tilde{\mathcal{M}}^{t / r}(s, r)$ are two real-valued, spherically symmetric distributions.

In view of the calculations in the following sections, it is most convenient to describe the singularity on the light cone by evaluating for fixed $r$ weakly in $s$. More precisely, we consider for any $0<s_{0}<r$ the integrals

$$
\int_{-s_{0}}^{s_{0}} s^{n} \tilde{\mathcal{M}}^{t / r}(s, r) d s, \quad n=0,1, \ldots
$$

which can be thought of as measuring the " $n^{\text {th }}$ moment" of the singularity. Here the lower limit of the integral is irrelevant because the integrand vanishes identically on the interval $\left(-s_{0}, 0\right)$; we only need to ensure to integrate over an open interval containing the origin. The upper limit of the integral is also not interesting because for different values of $s_{0}$, the corresponding integrals coincide up to a contribution which is completely determined by the well-known behavior of $\tilde{\mathcal{M}}$ away from the light cone. Therefore, we would like to take the limit $s_{0} \searrow 0$. However, since the distributions $\tilde{\mathcal{M}}^{t / r}$ have nonintegrable poles at $s=0$, the above integrals need not converge as $s_{0} \searrow 0$. But we can take the limit $s_{0} \searrow 0$ after subtracting indefinite integrals of the poles. More precisely, from (2.20) and (3.5) we have for small $s>0$ the expansions

$$
\begin{aligned}
\tilde{\mathcal{M}}^{0}(s, r) & =\frac{\mathfrak{m}_{3}}{4 r s^{2}}+\frac{\mathfrak{m}_{5}}{2 s}+\mathcal{O}(\log s) \\
\tilde{\mathcal{M}}^{r}(s, r) & =\frac{\mathfrak{m}_{3}}{4 r s^{2}}+\frac{\mathfrak{m}_{5}}{2 s}-\frac{\mathfrak{m}_{3}}{4 r^{2} s}+\mathcal{O}(\log s) .
\end{aligned}
$$

Thus we introduce the quantities

$$
\begin{aligned}
I^{0}(r) & =\lim _{s_{0} \searrow 0}\left\{\int_{-s_{0}}^{s_{0}} \tilde{\mathcal{M}}^{0}(s, r) d s+\frac{\mathfrak{m}_{3}}{4 r s_{0}}-\frac{\mathfrak{m}_{5}}{2} \log s_{0}\right\} \\
I^{r}(r) & =\lim _{s_{0} \searrow 0}\left\{\int_{-s_{0}}^{s_{0}} \tilde{\mathcal{M}}^{r}(s, r) d s+\frac{\mathfrak{m}_{3}}{4 r s_{0}}-\frac{\mathfrak{m}_{5}}{2} \log s_{0}+\frac{\mathfrak{m}_{3}}{4 r^{2}} \log s_{0}\right\} \\
J^{t / r}(r) & =\lim _{s_{0} \searrow 0}\left\{\int_{-s_{0}}^{s_{0}} s \tilde{\mathcal{M}}^{t / r}(s, r) d s-\frac{\mathfrak{m}_{3}}{4 r} \log s_{0}\right\} \\
K_{n}^{t / r}(r) & =\lim _{s_{0} \searrow 0} \int_{-s_{0}}^{s_{0}} s^{n} \tilde{\mathcal{M}}^{t / r}(s, r) d s, \quad n \geq 2 .
\end{aligned}
$$

These real-valued functions are well defined for all $r>0$. They have the nice property of being independent of all smooth contributions to $\tilde{\mathcal{M}}(\xi)$ as well as of the integrable poles of $\tilde{\mathcal{M}}(\xi)$. This makes it possible in what follows to work only with the expansion of $f$ as given by (3.5), thus avoiding the complicated formulas involving Bessel functions.

Lemma 3.2 The functions $K_{n}^{t / r}$ all vanish. Furthermore,

$$
I^{0}(r)=\frac{\mathfrak{m}_{3}}{8} \frac{1}{r^{2}}+\frac{\mathfrak{m}_{5}}{2} \log 2 r+\frac{\mathfrak{m}_{3}+c_{1}}{2}
$$




$$
\begin{aligned}
J^{0}(r) & =\frac{\mathfrak{m}_{3}}{4} \frac{\log 2 r}{r}+\frac{\mathfrak{m}_{3}-2 c_{0}}{8 r} \\
I^{r} & =I^{0}-\frac{1}{r} J^{0}, \quad J^{r}=J^{0} .
\end{aligned}
$$

Proof. The identities for the radial component follow from those for the time component simply using the Lorentz symmetry of $\tilde{\mathcal{M}}$. Namely, assume for the moment that the lemma holds for the time component. According to (2.21) and (3.8), we can write $\tilde{\mathcal{M}}^{t / r}$ as derivatives of a Lorentz invariant distribution $G:=\square F$,

$$
\tilde{\mathcal{M}}^{0}=\partial_{t} G\left(t^{2}-r^{2}\right), \quad \tilde{\mathcal{M}}^{r}=-\partial_{r} G\left(t^{2}-r^{2}\right) .
$$

Differentiating with the Leibniz rule, one sees that $r \tilde{\mathcal{M}}^{0}=t \tilde{\mathcal{M}}^{r}$, and thus

$$
\tilde{\mathcal{M}}^{r}=\frac{r}{r+s} \tilde{\mathcal{M}}^{0}=\left(1-\frac{s}{r}+\mathcal{O}\left(s^{2}\right)\right) \tilde{\mathcal{M}}^{0} .
$$

Integrating over $s$ yields (3.15) as well as the vanishing of (3.12) for the radial component.

It remains to consider the time component. Using (3.5) in (3.6), we obtain for $g$ the expansion

$$
g(z)=-\frac{\mathfrak{m}_{3}}{8} \log z+\frac{\mathfrak{m}_{5}}{16} z \log z+\frac{4 c_{0}-2 \mathfrak{m}_{3}+3 \mathfrak{m}_{5}}{32}+\frac{2 c_{1}+2 \mathfrak{m}_{3}-3 \mathfrak{m}_{5}}{32} z+\mathcal{O}\left(z^{2} \log z\right) .
$$

One possible way to proceed would be to regularize the poles of $g$, to compute the corresponding regularized $\tilde{\mathcal{M}}$ via $(2.21,2.22)$, to evaluate weakly in time and finally to remove the regularization (this method is well-suited for computer algebra). Here we describe a different method, which is a bit more elegant. We choose a test function $\eta \in C_{0}^{\infty}(\mathbb{R})$ which vanishes in a neighborhood of the origin. Due to spherical symmetry, we can omit the angular integrals. Then, applying the definition of weak derivatives to (2.21), we obtain

$\int_{0}^{\infty} r^{2} \eta(r) d r \int_{-s_{0}}^{s_{0}} d s s^{n} \tilde{\mathcal{M}}^{0}(t, r)=-\int_{0}^{\infty} r^{2} d r \int_{0}^{\infty} d s g\left(t^{2}-r^{2}\right) \square \partial_{t}\left(\eta(r) s^{n} \Theta\left(s_{0}-s\right)\right)$,

where the derivatives act on $\Theta\left(s_{0}-s\right)$ in the distributional sense, giving $\delta$ - and $\delta^{\prime}$-contributions supported on the hypersurface $s=s_{0}$ where $g$ is smooth. The derivatives of $\eta$ can be rewritten as directional derivatives $\partial_{t}+\partial_{r}$ tangential to the light cone, $\eta^{(k)}(r)=$ $\left(\partial_{t}+\partial_{r}\right)^{k} \eta(r)$. These directional derivatives may be integrated by parts because the resulting tangential derivatives of $g$ are again integrable. The resulting expression is of the general form

$$
\int_{0}^{\infty} r^{2} \eta(r) d r \int_{0}^{\infty} d s \cdots
$$

and justifies that $\tilde{\mathcal{M}}^{0}$ can indeed be evaluated for fixed $r$ weakly in $t$. Furthermore, going through the detailed calculation, one finds that

$$
\begin{gathered}
\int_{-s_{0}}^{s_{0}} s^{n} \tilde{\mathcal{M}}^{0}(s, r) d r=-2 n r^{-1} s^{n-1} g-4(n-2) s^{n} g^{\prime}+\left.4(t+r) s^{n+1} g^{\prime \prime}\right|_{s=s_{0}} \\
\quad+\int_{0}^{s_{0}}\left(2 n(n-1) r^{-1} s^{n-2} g+4 n\left(n-1+r^{-1} s\right) s^{n-1} g^{\prime}+4 n s^{1+n} g^{\prime \prime}\right) d s .
\end{gathered}
$$

The last integral vanishes in the limit $s_{0} \searrow 0$ for any $n \geq 0$. Furthermore, in the case $n \geq 2$ it is obvious that the boundary terms at $s_{0}$ tend to zero as $s_{0} \searrow 0$. In the cases $n=0,1$, the boundary terms diverge as $s_{0}$ tends to zero. But these divergences are exactly canceled by the counterterms in (3.9) and (3.11). Thus we can compute the limit $s_{0} \searrow 0$ to obtain the result. 


\section{The Momentum Cone Conditions}

In this section we explain a particular difficulty in proving Theorem 2.3 (3). Apart from illustrating the statement of our main theorem, our analysis will lead to conditions which will be important for the constructions of the subsequent sections. Let us assume for the moment that Theorem 2.3 (2) holds, i.e. suppose that we already know that $\mathcal{M}\left[A_{x y}^{\varepsilon}\right]$ converges as a distribution to $\tilde{\mathcal{M}}(\xi)$. This statement entails that, in the limit $\varepsilon \searrow 0, \mathcal{M}\left[A_{x y}^{\varepsilon}\right]$ develops singularities on the light cone as characterized by Lemma 3.2. If $P^{\varepsilon}(x, y)$ converged locally uniformly to a smooth function, we could immediately conclude that the product $\mathcal{M}\left[A_{x y}^{\varepsilon}\right] P^{\varepsilon}(x, y)$ converges in the distributional sense to $\tilde{\mathcal{M}}(\xi) P(x, y)$. Unfortunately, the situation is more difficult because $P(x, y)$ also has singularities on the light cone (see (3.1 3.3) ). As a consequence, we must expect that in the limit $\varepsilon \searrow 0$, the function $\mathcal{M}\left[A_{x y}^{\varepsilon}\right] P^{\varepsilon}(x, y)$ will develop singularities on the light cone, which do not even make sense as distributions. The main difficulty in proving Theorem 2.3 (3) is to show that, despite the divergences of the product $\mathcal{M}\left[A_{x y}^{\varepsilon}\right] P^{\varepsilon}(x, y)$ on the light cone, its Fourier transform has a well defined limit inside the lower mass cone, which coincides with (2.25).

The nature of this problem becomes clearer when working purely in momentum space. Similar to (2.25), we can rewrite the product in position space as a convolution in momentum space,

$$
\int \frac{d^{4} p}{(2 \pi)^{4}} \hat{\mathcal{M}}^{\varepsilon}(p) \hat{P}^{\varepsilon}(q-p) .
$$

We let $q$ be a fixed vector inside the lower mass cone. In contrast to the situation in Figure 1, $\hat{\mathcal{M}}^{\varepsilon}$ will not be supported inside the mass cone, because the regularization will in general give rise also to a contribution outside the mass cone. Furthermore, for $p^{0}$ on the order of the Planck energy, the support of $\hat{P}^{\varepsilon}$ may no longer lie on the mass hyperbolas, but might have a completely different form. For $p$ in any compact set, these regularization effects are not a problem, because the distributional convergences $\hat{\mathcal{M}}^{\varepsilon} \rightarrow \hat{\mathcal{M}}$ and $P^{\varepsilon} \rightarrow P$ ensure that for bounded $p$ and $q$ the effects of the regularization tend to zero as $\varepsilon \searrow 0$. However, the problem is to show that the contribution due to the regularization for large $p^{0}$ to the convolution integral (4.1) tends to zero as $\varepsilon \searrow 0$.

In order to develop a method for analyzing an integral of the form (4.1) for large $p^{0}$, we first make a few simplifications. First, we shall regularize only one factor and consider the product $\mathcal{M}\left[A_{x y}^{\varepsilon}\right] P(x, y)$ of the regularized $\mathcal{M}$ with the unregularized $P$. Thus $P$ is singular on the light cone, whereas $\mathcal{M}\left[A_{x y}^{\varepsilon}\right]$ is, due to the regularization, a smooth function. Furthermore, we assume that $\mathcal{M}\left[A_{x y}^{\varepsilon}\right]$ vanishes in a neighborhood of the light cone. This assumption seems to make the problem trivial, because as a consequence the product $\mathcal{M}\left[A_{x y}^{\varepsilon}\right] P(x, y)$ is even a smooth function. Nevertheless, this case is interesting because the singularities on the light cone will reappear as soon as we take the limit $\varepsilon \searrow 0$.

Moreover, we replace the mass shells of $P$ for large $p^{0}$ by one cone, whereas for $p$ in a compact set we are free to choose $P$ in a convenient way. Finally, we choose a reference frame such that $q=(-\Omega, \overrightarrow{0})$ with $\Omega>0$. We are thus led to the question of whether the integrals

$$
B^{\varepsilon}:=\int \frac{d^{4} p}{(2 \pi)^{4}} \hat{\mathcal{M}}^{\varepsilon}(p) \hat{H}(q-p)
$$

vanish in the limit $\varepsilon \searrow 0$, where $\hat{H}$ is a distribution whose support can be chosen for example as in Figure 2 (a) or (b). More specifically, in order to model the scalar and 


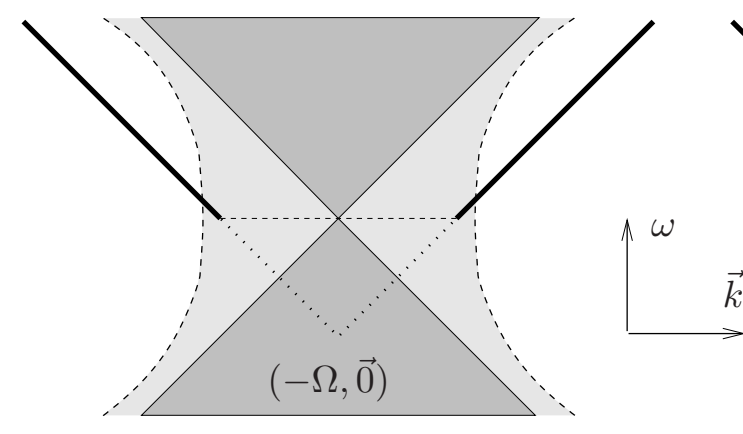

(a)

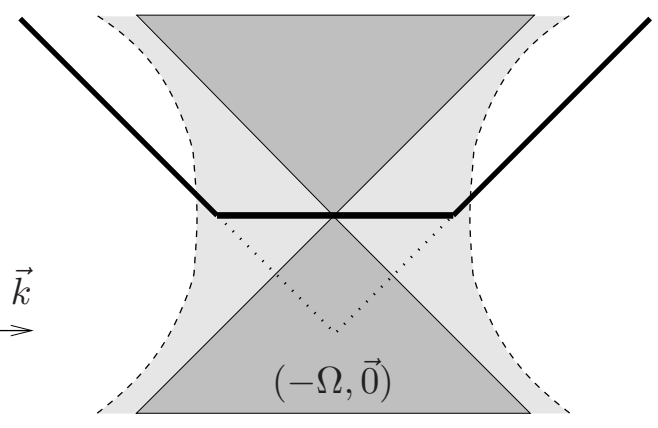

(b)

Figure 2: The convolution $\hat{\mathcal{M}}^{\varepsilon} * \hat{H}$ for different choices of $\hat{H}$.

vector components of $P$, we shall choose $\hat{H}$ equal to

$$
\left.\hat{H}_{\text {scal }}(k)=\delta\left(k^{2}\right) \Theta\left(-k^{0}-\Omega\right) \quad \text { (see Figure } 2(\mathrm{a})\right)
$$

and

$$
\begin{aligned}
\hat{H}_{\text {vect }}(k) & =\not k \delta\left(k^{2}\right) \Theta\left(-k^{0}-\Omega\right)-\frac{1}{2} \gamma^{0} \delta\left(-k^{0}-\Omega\right) \Theta\left(k^{2}\right) \\
& =\frac{1}{2} \not \not_{k}\left(\Theta\left(k^{2}\right) \Theta\left(-k^{0}-\Omega\right)\right) \quad \text { (see Figure 2 (b)), }
\end{aligned}
$$

respectively. This choice of $\hat{H}$ has the advantage that it will be possible to easily compute the Fourier integral of $\hat{H}$.

Despite all the simplifications made, the analysis of (4.2) for $H$ according to (4.3) and (4.4) will be helpful in order to understand under which conditions on $\mathcal{M}\left[A_{x y}^{\varepsilon}\right]$ we can expect that Theorem 2.3 (3) holds. We first bring the integral (4.2) into a more explicit form in position space.

Lemma 4.1 Suppose that the function $\mathcal{M}\left[A_{x y}^{\varepsilon}\right]$ vanishes in a neighborhood of the light cone. Then for $\hat{H}$ according to (4.3) and (4.4), the corresponding convolution integral (4.2) is given by

$$
\begin{aligned}
B_{\text {scal }}^{\varepsilon} & =\int \frac{d^{4} \xi}{16 \pi^{3}} \mathcal{M}\left[A_{x y}^{\varepsilon}\right]\left(\frac{e^{-i \Omega r}}{r(t+r)}-\frac{e^{i \Omega r}}{r(t-r)}\right) \\
B_{\text {vect }}^{\varepsilon} & =\int \frac{d^{4} \xi}{16 \pi^{3}}\left(\mathcal{M}\left[A_{x y}^{\varepsilon}\right] i \not g\right) \frac{1}{r} \frac{\partial}{\partial r}\left(\frac{e^{-i \Omega r}}{r(t+r)}-\frac{e^{i \Omega r}}{r(t-r)}\right),
\end{aligned}
$$

respectively, where again $\xi=(t, \vec{x})$ and $r=|\vec{x}|$.

Proof. We first consider the function $\hat{H}_{\text {scal }}$. Its Fourier transform can be computed in polar coordinates,

$$
\begin{aligned}
H_{\text {scal }}(\xi) & =\frac{1}{(2 \pi)^{3}} \int_{-\infty}^{-\Omega} d \omega e^{i \omega t} \int_{0}^{\infty} p^{2} d p \delta\left(\omega^{2}-p^{2}\right) \int_{-1}^{1} d \cos \vartheta e^{-i p r \cos \vartheta} \\
& =\frac{i}{8 \pi^{3} r} \int_{-\infty}^{-\Omega} d \omega e^{i \omega t} \int_{0}^{\infty} p d p \delta\left(\omega^{2}-p^{2}\right)\left(e^{-i p r}-e^{i p r}\right)
\end{aligned}
$$




$$
\begin{aligned}
& =\frac{i}{16 \pi^{3} r} \int_{-\infty}^{-\Omega} d \omega\left(e^{i \omega(t+r)}-e^{i \omega(t-r)}\right) \\
& =\frac{1}{16 \pi^{3}} \frac{1}{r}\left[e^{-i \Omega(t+r)}\left(\frac{\mathrm{PP}}{t+r}+i \pi \delta(t+r)\right)-e^{-i \Omega(t-r)}\left(\frac{\mathrm{PP}}{t-r}+i \pi \delta(t-r)\right)\right] .
\end{aligned}
$$

Using Plancherel's theorem, we can rewrite (4.2) in position space as

$$
B^{\varepsilon}=\int e^{i \Omega t} \mathcal{M}\left[A_{x y}^{\varepsilon}\right] H(\xi) d^{4} \xi .
$$

Substituting the above formula for $H_{\text {scal }}(\xi)$ and using that $M\left[A_{x y}^{\varepsilon}\right]$ vanishes in a neighborhood of the light cone, we obtain (4.5). The identity (4.6) follows similarly from the calculation

$$
\begin{aligned}
H_{\mathrm{vect}}(\xi) & =\int \frac{d^{4} k}{32 \pi^{4}} \not \partial_{k}\left(\Theta\left(k^{2}\right) \Theta\left(-k^{0}-\Omega\right)\right) e^{i k \xi} \\
& =-i \not \varnothing \int \frac{d^{4} k}{32 \pi^{4}} \Theta\left(k^{2}\right) \Theta\left(-k^{0}-\Omega\right) e^{i k \xi} \\
& =\frac{\not}{16 \pi^{3} r} \int_{-\infty}^{-\Omega} d \omega e^{i \omega t} \int_{0}^{|\omega|} p d p\left(e^{-i p r}-e^{i p r}\right) \\
& =\frac{i \not}{16 \pi^{3} r} \frac{\partial}{\partial r} \int_{-\infty}^{-\Omega} d \omega e^{i \omega t} \int_{0}^{|\omega|}\left(e^{-i p r}+e^{i p r}\right) d p \\
& =-\frac{\not}{16 \pi^{3} r} \frac{\partial}{\partial r}\left\{\frac{1}{r} \int_{-\infty}^{-\Omega} d \omega\left(e^{i \omega(t+r)}-e^{i \omega(t-r)}\right)\right\} \\
& =\frac{i \not \phi}{16 \pi^{3} r} \frac{\partial}{\partial r}\left\{\left.\frac{1}{r} e^{-i \Omega u}\left(\frac{\mathrm{PP}}{u}+i \pi \delta(u)\right)\right|_{u=t-r} ^{u=t+r}\right\} .
\end{aligned}
$$

Let us discuss the result of this lemma, first in the case of a Lorentz invariant regularization, which for convenience we write in the form

$$
\mathcal{M}\left[A_{x y}^{\varepsilon}\right]=\frac{1}{2}\left(\not \partial g_{\varepsilon}\left(\xi^{2}\right)\right) \epsilon\left(\xi^{0}\right)=\not g g_{\varepsilon}^{\prime}\left(\xi^{2}\right) \epsilon\left(\xi^{0}\right)
$$

with a smooth real function $g_{\varepsilon}$ which vanishes if $\xi^{2}<0$. Then the argument leading to (2.24) still holds and thus $\operatorname{supp} \hat{\mathcal{M}}\left[A^{\varepsilon}\right] \subset \overline{\mathcal{C}}$. As a consequence, a support argument (see Figure 21) shows that the integral (4.2) vanishes. It is instructive to reproduce this fact using the formulas of Lemma 4.1. We first collect the terms for a fixed phase factor $e^{-i \Omega r}$ in (4.5). Due to spherical symmetry, the radial component $\sim \gamma^{r}$ of (4.9) drops out. Using the symmetry under time reflections, we can write the time integral of the remaining component $\sim \gamma^{0}$ as

$$
e^{-i \Omega r} \int_{-\infty}^{\infty} \epsilon(t) g_{\varepsilon}^{\prime}\left(\xi^{2}\right) \frac{t}{r(t+r)} d t=-e^{-i \Omega r} \int_{0}^{\infty} \frac{g_{\varepsilon}^{\prime}\left(\xi^{2}\right)}{\xi^{2}} 2 t d t=-e^{-i \Omega r} \int_{-r^{2}}^{\infty} \frac{g_{\varepsilon}^{\prime}(z)}{z} d z
$$

where in the last step we introduced the "Lorentz invariant" integration variable $z(t)=$ $t^{2}-r^{2}$. Since $g_{\varepsilon}^{\prime}$ vanishes for negative $z$, it is obvious that the last integral is equal to a constant $c$, independent of $r$ and the angular variables. Similarly, for the contribution $\sim \gamma^{0}$ involving the phase factor $e^{i \Omega r}$, the time integral is computed to be

$$
-e^{i \Omega r} \int_{-\infty}^{\infty} \epsilon(t) g_{\varepsilon}^{\prime}\left(\xi^{2}\right) \frac{t}{r(t-r)} d t=-e^{i \Omega r} \int_{-r^{2}}^{\infty} \frac{g_{\varepsilon}^{\prime}(z)}{z} d z
$$


and the last integral again equals $c$. Adding the obtained terms involving the phase factors $e^{-i \Omega r}$ and $e^{i \Omega r}$, we can carry out the radial integral to get

$$
-c \int_{0}^{\infty} r^{2}\left(e^{-i \Omega r}+e^{i \Omega r}\right) d r=-c \int_{-\infty}^{\infty} r^{2} e^{-i \Omega r} d r=c 2 \pi \delta^{\prime \prime}(\Omega)=0,
$$

where in the last step we used that $\Omega \neq 0$. This explains why $B_{\text {scal }}^{\varepsilon}$ vanishes. For $B_{\text {vect }}^{\varepsilon}$, we likewise collect the terms involving the phase factor $e^{-i \Omega r}$ in (4.6). Then we can carry out the time integral as follows,

$$
\begin{aligned}
& \int_{-\infty}^{\infty} i \xi^{2} g_{\varepsilon}^{\prime}\left(\xi^{2}\right) \epsilon(t) \frac{1}{r} \frac{\partial}{\partial r}\left(\frac{e^{-i \Omega r}}{r(t+r)}\right) d t \\
& =e^{-i \Omega r} \int_{-\infty}^{\infty} i \xi^{2} g_{\varepsilon}^{\prime}\left(\xi^{2}\right) \epsilon(t)\left[-\frac{i \Omega r+1}{r^{3}(t+r)}-\frac{1}{r^{2}(t+r)^{2}}\right] d t \\
& =e^{-i \Omega r} \int_{0}^{\infty} i \xi^{2} g_{\varepsilon}^{\prime}\left(\xi^{2}\right)\left[-\frac{(i \Omega r+1) 2 t}{r^{3} \xi^{2}}+\frac{4 t}{r \xi^{4}}\right] d t \\
& =e^{-i \Omega r} \frac{\Omega r-i}{r^{3}} \int_{-r^{2}}^{\infty} g_{\varepsilon}^{\prime}(z) d z+e^{-i \Omega r} \frac{2 i}{r} \int_{-r^{2}}^{\infty} \frac{g_{\varepsilon}^{\prime}(z)}{z} d z
\end{aligned}
$$

The first integral in the last line can be evaluated and is seen to vanish (note that, in view of the representation (2.21, 2.22), we know that $g(\infty)=0$ ). The second integral is again equal to a constant $c$, independent of $r$ and the angular variables. As a consequence, it vanishes similar to (4.10) after adding the corresponding contribution involving the phase factor $e^{i \Omega r}$ and carrying out the radial integral

$$
2 i c \int_{0}^{\infty} r^{2}\left(\frac{e^{-i \Omega r}}{r}-\frac{e^{i \Omega r}}{r}\right) d r=2 i c \int_{-\infty}^{\infty} r e^{-i \Omega r} d r=-2 c 2 \pi \delta^{\prime}(\Omega)=0 .
$$

To summarize, if $\mathcal{M}\left[A_{x y}^{\varepsilon}\right]$ is Lorentz invariant, the time integrals can be written in an invariant form using the measure $d z=2 t d t$. Then the radial integral becomes the Fourier transform of a polynomial, giving a distribution supported at $\Omega=0$.

If conversely $\mathcal{M}\left[A_{x y}^{\varepsilon}\right]$ is not Lorentz invariant, it is impossible to rewrite the time integrals in the above invariant form. As a consequence, they will in general depend on $r$ in a complicated way, and the $r$-integral will no longer be supported at $\Omega=0$. One might even conjecture that the vanishing of (4.5) and (4.6) implies the Lorentz invariance of $\mathcal{M}\left[A_{x y}^{\varepsilon}\right]$. However, this conjecture is false, as one sees easily in examples such as

$$
\mathcal{M}\left[A_{x y}^{\varepsilon}\right]=\not \partial\left(r^{2 n} g_{\varepsilon}\left(t^{2}-r^{2}\right)\right) \epsilon(t),
$$

which obviously violate Lorentz invariance, but where the resulting time integrals are nevertheless polynomials in $r$.

Let us work out systematically under which conditions the integrals (4.5) and (4.6) vanish. In order to avoid technical complications, we shall assume that $\mathcal{M}\left[A_{x y}^{\varepsilon}\right]$ is strictly causal in the sense that it is supported inside the interior light cone. Our analysis is simplified considerably by our assumption that $\mathcal{M}\left[A_{x y}^{\varepsilon}\right]$ converges as a distribution to $\tilde{\mathcal{M}}$. Namely, whenever we have an expression of the form $\mathcal{M}\left[A_{x y}^{\varepsilon}\right]$ times a smooth function, this expression converges as $\varepsilon \searrow 0$ in the distributional sense, and we could easily compute its limit. Therefore, we may disregard such so-called distributional expressions. In order to explain how to compute modulo distributional expressions, let us simplify the expression (4.5) for $B_{\text {scal }}^{\varepsilon}$. Using the convention

$$
\mathcal{M}(t,-r, \vartheta, \varphi)=\mathcal{M}(t, r, \vartheta+\pi, \varphi),
$$


we may extend the function $\mathcal{M}\left[A_{x y}^{\varepsilon}\right]$ to negative values of $r$ (thus for fixed $\vartheta$ and $\varphi$, the curve $r \in \mathbb{R}$ is a straight line through the origin). Then we can rewrite the radial integral as

$$
\int_{0}^{\infty} r^{2} d r\left(\frac{e^{-i \Omega r}}{r(t+r)}-\frac{e^{i \Omega r}}{r(t-r)}\right) \mathcal{M}\left[A_{x y}^{\varepsilon}\right]=\int_{-\infty}^{\infty} e^{-i \Omega r} \frac{\mathcal{M}\left[A_{x y}^{\varepsilon}\right]}{t+r} r d r
$$

Now our task is to analyze the time integral of $\mathcal{M}\left[A_{x y}^{\varepsilon}\right] /(t+r)$. Since $\mathcal{M}\left[A_{x y}^{\varepsilon}\right]$ is strictly causal, we only need to consider the two regions $t>|r|$ and $t<-|r|$. We can even restrict attention to the region where the factor $(t+r)^{-1}$ has a pole,

$$
\int_{-\infty}^{\infty} \frac{\mathcal{M}\left[A_{x y}^{\varepsilon}\right]}{t+r} d t=\mp \int_{0}^{\mp \infty} \frac{\mathcal{M}\left[A_{x y}^{\varepsilon}\right]}{t+r} d t+\text { (distributional) }
$$

where we distinguished the two cases $r>0$ and $r<0$, respectively. Furthermore, we may add distributional terms; in particular,

$$
\begin{aligned}
\int_{0}^{\mp \infty} \frac{\mathcal{M}\left[A_{x y}^{\varepsilon}\right]}{t+r} d t & =\int_{0}^{\mp \infty} \mathcal{M}\left[A_{x y}^{\varepsilon}\right]\left(\frac{1}{t+r}-\frac{1}{t-r}\right) d t+\text { (distributional) } \\
& =-2 r \int_{0}^{\mp \infty} \frac{\mathcal{M}\left[A_{x y}^{\varepsilon}\right]}{t^{2}-r^{2}} d t+\text { (distributional) } .
\end{aligned}
$$

In this way, we have rewritten (4.5) as

$$
\begin{aligned}
16 \pi^{3} B_{\text {scal }}^{\varepsilon}= & 2 \int_{0}^{2 \pi} d \varphi \int_{-1}^{1} d \cos \vartheta \int_{-\infty}^{\infty} d r e^{-i \Omega r} r^{2} \epsilon(r) \int_{0}^{-\epsilon(r) \infty} \frac{\mathcal{M}\left[A_{x y}^{\varepsilon}\right]}{t^{2}-r^{2}} d t \\
& +(\text { distributional }) .
\end{aligned}
$$

In the omitted distributional terms, we may replace $\mathcal{M}\left[A_{x y}^{\varepsilon}\right]$ by a Lorentz invariant regularization of the form (4.9). As explained above, for such a Lorentz invariant regularization, the time integral in (4.14) is independent of $r$, and thus the radial integral gives zero (note that the factor $\&$ in (4.9) contains a factor $t$ which can be combined with the $d t$ in (4.14) to the Lorentz invariant measure $d z$ ). Hence for the Lorentz invariant distribution, the integrals in (4.14) and the distributional terms both tend to zero as $\varepsilon \searrow 0$. Again using that the limit $\varepsilon \searrow 0$ of the distributional terms is independent of the regularization, we conclude that $B_{\text {scal }}^{\varepsilon}$ vanishes as $\varepsilon \searrow 0$ if and only if the integrals in (4.14) tend to zero in this limit. This leads us to impose that the time integral in (4.14) should be a polynomial in $r$.

Definition 4.2 Suppose that $\left(P^{\varepsilon}\right)_{\varepsilon>0}$ is a family of regularized fermionic projectors, such that $P^{\varepsilon} \rightarrow P$ and $\mathcal{M}\left[A_{x y}^{\varepsilon}\right] \rightarrow \tilde{\mathcal{M}}$ as distributions. The functions $\mathcal{M}\left[A_{x y}^{\varepsilon}\right]$ satisfy the scalar momentum cone conditions if there is a finite number of constants $\left(c_{i}\right)_{i=0,1, \ldots}$ (which may depend on the angular variables, but are independent of $r$ ) such that for every $r>0$,

$$
\int_{0}^{ \pm \infty} \frac{\mathcal{M}\left[A_{x y}^{\varepsilon}\right]( \pm r)}{t^{2}-r^{2}} d t \pm \frac{c_{0}(\varepsilon)}{r^{2}}+\frac{c_{1}(\varepsilon)}{r} \pm c_{2}(\varepsilon)+c_{3}(\varepsilon) r+\cdots \stackrel{\varepsilon \searrow 0}{\longrightarrow} 0,
$$

where $\mathcal{M}$ is defined for negative $r$ by 4.12). 
The function $B_{\text {vect }}^{\varepsilon}$, (4.6), can be handled in a similar way, where it is most convenient to integrate the additional radial derivative by parts. We first rewrite the radial integral as follows,

$$
\begin{aligned}
\int_{0}^{\infty} & r^{2} d r\left(\mathcal{M}\left[A_{x y}^{\varepsilon}\right] i \not\right)(r) \frac{1}{r} \frac{\partial}{\partial r}\left(\frac{e^{-i \Omega r}}{r(t+r)}-\frac{e^{i \Omega r}}{r(t-r)}\right) \\
= & \int_{-\infty}^{\infty} r d r\left(\mathcal{M}\left[A_{x y}^{\varepsilon}\right] i \not\right)(r) \frac{\partial}{\partial r}\left(\frac{e^{-i \Omega r}}{r(t+r)}\right) \\
= & -\int_{-\infty}^{\infty} \frac{\partial}{\partial r}\left(r\left(\mathcal{M}\left[A_{x y}^{\varepsilon}\right] i \not\right)(r)\right) \frac{e^{-i \Omega r}}{t+r} \frac{d r}{r} .
\end{aligned}
$$

Computing modulo distributional expressions, the resulting time integral can be written in the two cases $r>0$ and $r<0$ as

$$
\begin{aligned}
\int_{-\infty}^{\infty} & \frac{\partial}{\partial r}\left(r\left(\mathcal{M}\left[A_{x y}^{\varepsilon}\right] i \not\right)(r)\right) \frac{1}{t+r} d t \\
= & \mp \int_{0}^{\mp \infty} \frac{\partial}{\partial r}\left(r\left(\mathcal{M}\left[A_{x y}^{\varepsilon}\right] i \not\right)(r)\right) \frac{1}{t+r} d t+(\text { distributional) } \\
= & \mp 2 \int_{0}^{\mp \infty} \frac{\partial}{\partial r}\left(r\left(\mathcal{M}\left[A_{x y}^{\varepsilon}\right] i \not\right)(r)\right) \frac{1}{t^{2}-r^{2}} t d t+\text { (distributional). }
\end{aligned}
$$

A short calculation shows that for the Lorentz invariant regularization (4.9), the last integral is a polynomial in $r$ of degree two, and thus the radial integral vanishes. We thus end up with the following condition.

Definition 4.3 Suppose that $\left(P_{x y}^{\varepsilon}\right)_{\varepsilon>0}$ is a family of regularized fermionic projectors, such that $P^{\varepsilon} \rightarrow P$ and $\mathcal{M}\left[A_{x y}^{\varepsilon}\right] \rightarrow \tilde{\mathcal{M}}$ as distributions. The functions $\mathcal{M}\left[A_{x y}^{\varepsilon}\right]$ satisfy the vector momentum cone conditions if there is a finite number of constants $\left(c_{i}\right)_{i=0,1, \ldots}$ (which may depend on the angular variables, but are independent of $r$ ) such that for every $r>0$,

$$
\left.\int_{0}^{ \pm \infty} \frac{\partial}{\partial r}\left(r \mathcal{M}\left[A_{x y}^{\varepsilon}\right]( \pm r) i \not\right)\right) \frac{t d t}{t^{2}-r^{2}}+c_{0}(\varepsilon) r \pm c_{1}(\varepsilon) r^{2}+c_{2}(\varepsilon) r^{3}+\cdots \stackrel{\varepsilon \searrow_{0}}{\longrightarrow} 0 .
$$

We close this section with a few remarks. For clarity, we mention that, since $P(x, y)$ is the sum of a scalar and vector component, we do not need to satisfy the conditions of Definitions 4.2 and 4.3 separately; it suffices to satisfy a certain linear combination of these conditions.

In the previous analysis we worked in a particular reference frame, where the $t$ coordinate pointed in the direction of the vector $q$. Nevertheless, the momentum cone conditions are the same in any other reference frame, as the following consideration shows. In a general reference frame, the vector $q$ lies in the interior of the lower mass cone and can thus be written as $q=(-\Omega, \vec{K})$ with $|\vec{K}|<\Omega$. On a compact set, $\hat{H}_{\text {scal }}$ and $\hat{H}_{\text {vect }}$ are again arbitrary. It is most convenient to choose

$$
\begin{aligned}
\hat{H}_{\text {scal }}(k) & =\delta\left(\left(k^{0}\right)^{2}-(\vec{k}-\vec{K})^{2}\right) \Theta\left(-k^{0}-\Omega\right) \\
\hat{H}_{\text {vect }}(k) & =\frac{1}{2} \not \partial_{k}\left(\Theta\left(\left(k^{0}\right)^{2}-(\vec{k}-\vec{K})^{2}\right) \Theta\left(-k^{0}-\Omega\right)\right),
\end{aligned}
$$

because these distributions differ from (4.3, 4.4) simply by a shift in the spatial direction $\vec{K}$. Then the corresponding convolution integrals (4.2) are obtained from the formulas of 


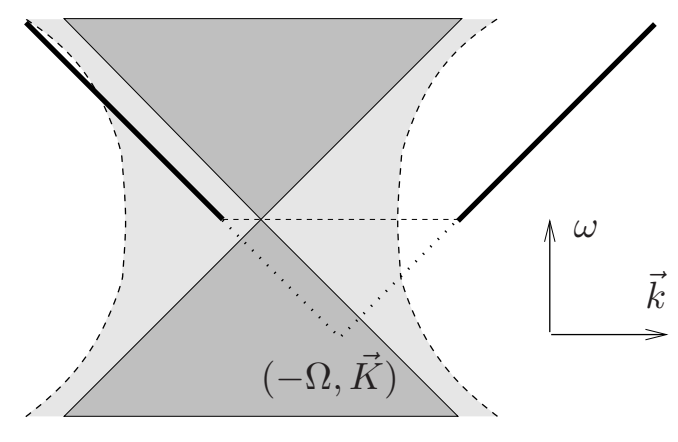

(a)

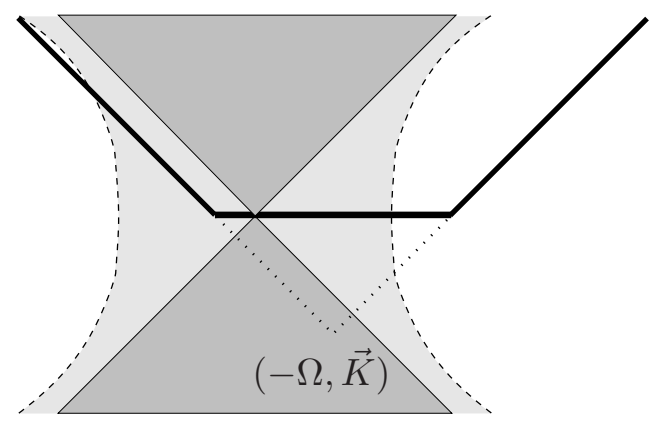

(b)

Figure 3: The convolution integral $\hat{\mathcal{M}}^{\varepsilon} * \hat{H}$ in a general reference frame.

Lemma 4.1 simply by inserting the phase factor $e^{-i \vec{K} \vec{\xi}}$ into the integrands in (4.5, 4.6) (see also Figure 3). As the additional phase $\vec{K} \vec{\xi}$ is time independent and linear in $r$, it has no effect on the subsequent arguments. In particular, the formulas (4.14, 4.16) remain valid if we only replace $\Omega$ by $\tilde{\Omega}=\Omega+|\vec{K}| \cos \alpha>0$, where $\alpha$ is the angle between $\vec{K}$ and $\vec{\xi}$. Since $\alpha$ is fixed in our argument, we again obtain the conditions of Definitions 4.2 and 4.3 .

We finally recall that the momentum cone conditions were derived under the assumption that $P$ is not regularized. Furthermore, we made the simplification of working with a cone instead of mass hyperbolas (compare Figures 1 and 3 ). These assumptions and simplifications still need to be justified. Indeed, we will have to slightly modify the momentum cone conditions in order to take these additional effects into account. We postpone these more technical aspects to Sections 8 and 11.

\section{The Spherically Symmetric Regularization}

For the regularized fermionic projector of the vacuum we take the general spherically symmetric ansatz

$$
\begin{aligned}
P^{\varepsilon}(x, y)= & \int \frac{d^{4} k}{(2 \pi)^{4}} \sum_{\beta=1}^{3} \Theta(-\omega) \delta\left(|\vec{k}|-K_{\beta}(\omega)\right) \frac{1}{2|\vec{k}|} \\
& \times 16 \pi^{3}\left(\not k g_{\beta}(\omega)+\gamma^{0} f_{\beta}(\omega)+h_{\beta}(\omega)\right) e^{-i k(x-y)},
\end{aligned}
$$

where $\omega \equiv k^{0}$, and $K_{\beta}, g_{\beta}, f_{\beta}$ and $h_{\beta}$ are positive functions. The fermionic projector without regularization (2.11) is recovered by setting

$$
\begin{aligned}
K_{\beta}(\omega) & =\sqrt{\omega^{2}-m_{\beta}^{2}} \\
g_{\beta}(\omega) & =\frac{1}{16 \pi^{3}}, \quad f_{\beta}(\omega)=0, \quad h_{\beta}(\omega)=\frac{m_{\beta}}{16 \pi^{3}} .
\end{aligned}
$$

For the regularization, we will always choose functions $g_{\beta}, f_{\beta}$ and $h_{\beta}$ which decay in $\omega$ on the scale $\varepsilon^{-1}$. Clearly, the ansatz (5.1) is a special case of (2.12, 2.13); in particular, the fermionic projector is homogeneous and has a vector-scalar structure. Furthermore, $P^{\varepsilon}(k)$ is a sum of distributions supported on the hypersurfaces $|\vec{k}|=K_{\beta}(\omega)$. This property is called that the fermionic projector is composed of surface states. Another property of 
potential interest is that the surface states should be half occupied (see [6, §4.4]); in our setting this condition is equivalent to the following equation,

$$
\left(\omega g_{\beta}+f_{\beta}\right)^{2}-\left(K_{\beta} g_{\beta}\right)^{2}=h_{\beta}^{2} .
$$

The condition of half occupied surface states will be discussed in Remark 13.1

Let us bring the ansatz (5.1) into a more convenient form. First, we can use the spherical symmetry as well as the $\delta$-distribution $\delta\left(|\vec{k}|-K_{\beta}(\omega)\right)$ to reduce to one-dimensional Fourier transforms.

Lemma 5.1 The fermionic projector (5.1) has the representation

$$
\begin{aligned}
P^{\varepsilon}(x, y)= & \sum_{\beta=1}^{3} \frac{i}{r} \int_{\Xi_{\beta}} d \omega h_{\beta}(\omega) e^{i \omega t}\left(e^{-i K_{\beta}(\omega) r}-e^{i K_{\beta}(\omega) r}\right) \\
& +\gamma^{0} \sum_{\beta=1}^{3} \frac{i}{r} \int_{\Xi_{\beta}} d \omega\left(f_{\beta}(\omega)+\omega g_{\beta}(\omega)\right) e^{i \omega t}\left(e^{-i K_{\beta}(\omega) r}-e^{i K_{\beta}(\omega) r}\right) \\
& -\gamma^{r} \sum_{\beta=1}^{3} \frac{1}{r^{2}} \int_{\Xi_{\beta}} d \omega g_{\beta}(\omega) e^{i \omega t}\left(e^{-i K_{\beta}(\omega) r}-e^{i K_{\beta}(\omega) r}\right) \\
& -\gamma^{r} \sum_{\beta=1}^{3} \frac{i}{r} \int_{\Xi_{\beta}} d \omega K_{\beta}(\omega) g_{\beta}(\omega) e^{i \omega t}\left(e^{-i K_{\beta}(\omega) r}+e^{i K_{\beta}(\omega) r}\right),
\end{aligned}
$$

where $\Xi_{\beta}$ are the sets

$$
\Xi_{\beta}:=\left\{\omega<0 \text { with } K_{\beta}(\omega)>0\right\}
$$

Proof. For notational convenience, we consider one Dirac sea and omit the index $\beta$. Choosing polar coordinates

$$
k=(\omega, p \cos \vartheta, p \sin \vartheta \cos \varphi, p \sin \vartheta \sin \varphi), \quad y-x=(t, r, 0,0)
$$

(thus $\vartheta$ is the angle between the vectors $\vec{k}$ and $\vec{y}-\vec{x}$ ), we obtain

$$
\begin{aligned}
\int & \frac{d^{4} k}{(2 \pi)^{4}} \Theta(-\omega) \delta(|\vec{k}|-K(\omega)) \frac{1}{2|\vec{k}|} 16 \pi^{3} h(\omega) e^{-i k(x-y)} \\
= & \int_{-\infty}^{0} d \omega h(\omega) e^{i \omega t} \int_{0}^{\infty} p d p \delta(p-K(\omega)) \int_{-1}^{1} d \cos \vartheta e^{-i p r \cos \vartheta} \\
& =\frac{i}{r} \int_{-\infty}^{0} d \omega h(\omega) e^{i \omega t} \int_{0}^{\infty} d p \delta(p-K(\omega))\left(e^{-i p r}-e^{i p r}\right) \\
& =\frac{i}{r} \int_{\Xi} d \omega h(\omega) e^{i \omega t}\left(e^{-i K(\omega) r}-e^{i K(\omega) r}\right) .
\end{aligned}
$$

Similarly,

$$
\begin{aligned}
& \int \frac{d^{4} k}{(2 \pi)^{4}} \Theta(-\omega) \delta(|\vec{k}|-K(\omega)) \frac{1}{2|\vec{k}|} 16 \pi^{3} \gamma^{0} f(\omega) e^{-i k(x-y)} \\
& =\gamma^{0} \frac{i}{r} \int_{\Xi} d \omega f(\omega) e^{i \omega t}\left(e^{-i K(\omega) r}-e^{i K(\omega) r}\right)
\end{aligned}
$$


and

$$
\begin{aligned}
\int \frac{d^{4} k}{(2 \pi)^{4}} \Theta(-\omega) \delta(|\vec{k}|-K(\omega)) \frac{1}{2|\vec{k}|} 16 \pi^{3} \not k g(\omega) e^{-i k(x-y)} \\
=\left(-i \gamma^{0} \partial_{t}-i \gamma^{r} \partial_{r}\right) \int \frac{d^{4} k}{(2 \pi)^{4}} \Theta(-\omega) \delta(|\vec{k}|-K(\omega)) \frac{1}{2|\vec{k}|} 16 \pi^{3} g(\omega) e^{-i k(x-y)} \\
=\quad\left(-i \gamma^{0} \partial_{t}-i \gamma^{r} \partial_{r}\right) \frac{i}{r} \int_{\Xi} d \omega g(\omega) e^{i \omega t}\left(e^{-i K(\omega) r}-e^{i K(\omega) r}\right) \\
=\gamma^{0} \frac{i}{r} \int_{\Xi} d \omega \omega g(\omega) e^{i \omega t}\left(e^{-i K(\omega) r}-e^{i K(\omega) r}\right) \\
\quad-\gamma^{r} \frac{1}{r^{2}} \int_{\Xi} d \omega g(\omega) e^{i \omega t}\left(e^{-i K(\omega) r}-e^{i K(\omega) r}\right) \\
\quad-\gamma^{r} \frac{i}{r} \int_{\Xi} d \omega K(\omega) g(\omega) e^{i \omega t}\left(e^{-i K(\omega) r}+e^{i K(\omega) r}\right)
\end{aligned}
$$

Adding these terms gives the result.

In order to further simplify the representation for $P^{\varepsilon}(x, y)$, we observe that without a regularization, the expansion of (5.2)

$$
\sqrt{\omega^{2}-m_{\beta}^{2}}=|\omega|\left(1+\mathcal{O}\left(\frac{m_{\beta}^{2}}{\omega^{2}}\right)\right)
$$

shows that the functions $K_{\beta}(\omega)$ and $|\omega|$ come asymptotically close for large $|\omega|$ and coincide in the massless case $m_{\beta}=0$. Since the regularization effects come into play only for large energies, we can expect that even if a regularization is present, the function

$$
\alpha_{\beta}:=|\omega|-K_{\beta}(\omega)
$$

should be small for large $|\omega|$. Therefore, it is useful to expand in powers of $\alpha$ as follows,

$$
e^{i K_{\alpha}(\omega) r}=e^{-i \omega r} e^{-i \alpha_{\beta}(\omega) r}=e^{-i \omega r} \sum_{k=0}^{\infty} \frac{\left(-i \alpha_{\beta}(\omega) r\right)^{k}}{k !}
$$

(note that $\omega$ is always negative). Motivated by the fact that $\alpha_{\beta}$ vanishes in the unregularized massless case, we refer to (5.6) as the mass expansion. It is unpleasant that Lemma 5.1 involves factors $e^{+i K_{\beta} r}$ and $e^{-i K_{\beta} r}$, making the resulting formulas rather messy. It is therefore convenient to introduce the following short notation. Taking the complex conjugate of the representation of Lemma 5.1, one sees that the fermionic projector is invariant under the transformations

$$
P^{\varepsilon}(x, y) \rightarrow \overline{P^{\varepsilon}(x, y)}, \quad t \rightarrow-t, \quad \gamma^{r} \rightarrow-\gamma^{r},
$$

which we call a PCT-transformation (in analogy to the usual parity, charge and time transformation; actually, in our context this invariance is nothing but the Hermiticity condition $P(x, y)^{*}=P(y, x)$ expressed in polar coordinates). A PCT-transformation converts the terms involving the factors $e^{-i K_{\beta} r}$ and $e^{i K_{\beta} r}$ into each other. Therefore, we can in what follows consider only the terms involving $e^{i K_{\beta} t}$ and refer to the terms involving $e^{-i K_{\beta} t}$ by "PCT". 
Lemma 5.2 (mass expansion) The fermionic projector (5.1) has the representation

$$
\begin{aligned}
P^{\varepsilon}(x, y)= & \sum_{\beta=1}^{3} \frac{1}{r} \sum_{k=0}^{\infty} \frac{(-i)^{k+1} r^{k}}{k !} \int_{\Xi_{\beta}} d \omega h_{\beta} \alpha_{\beta}^{k} e^{i \omega s} \\
& +\gamma^{0} \sum_{\beta=1}^{3} \frac{1}{r} \sum_{k=0}^{\infty} \frac{(-i)^{k+1} r^{k}}{k !} \int_{\Xi_{\beta}} d \omega(f+\omega g) \alpha_{\beta}^{k} e^{i \omega s} \\
& -\gamma^{r} \sum_{\beta=1}^{3} \frac{1}{r} \sum_{k=0}^{\infty} \frac{(-i)^{k+1} r^{k}}{k !} \int_{\Xi_{\beta}} d \omega\left(\omega+i \frac{k-1}{r}\right) g \alpha_{\beta}^{k} e^{i \omega s}+(P C T) .
\end{aligned}
$$

Proof. From Lemma 5.1 we get

$$
\begin{aligned}
P^{\varepsilon}(x, y)= & -\sum_{\beta=1}^{3} \frac{i}{r} \int_{\Xi_{\beta}} d \omega h_{\beta} e^{i \omega s} e^{-i \alpha_{\beta} r} \\
& -\gamma^{0} \sum_{\beta=1}^{3} \frac{i}{r} \int_{\Xi_{\beta}} d \omega\left(f_{\beta}+\omega g_{\beta}\right) e^{i \omega s} e^{-i \alpha_{\beta} r} \\
& +\gamma^{r} \sum_{\beta=1}^{3} \frac{1}{r^{2}} \int_{\Xi_{\beta}} d \omega g_{\beta} e^{i \omega s} e^{-i \alpha_{\beta} r} \\
& -\gamma^{r} \sum_{\beta=1}^{3} \frac{i}{r} \int_{\Xi_{\beta}} d \omega\left(-\omega-\alpha_{\beta}\right) g_{\beta} e^{i \omega s} e^{-i \alpha_{\beta} r}+(\mathrm{PCT})
\end{aligned}
$$

and expand in powers of $\alpha_{\beta}$.

Before going on, we briefly describe how our spherically symmetric regularization is related to the general formulas for the regularized fermionic projector as derived in [6, Chapter 4]. In order to get a connection to the general form of the vector component as considered in [6, §4.4], we consider the fermionic projector near the future light cone $t=r$. Then the "PCT"-terms involve the oscillatory phase factors $e^{i \omega(t+r)}$, and the corresponding contributions to the fermionic projector are smooth. Such terms were not considered in [6, §4], and therefore we also leave them out here. Setting $s=t-r$ and

$$
\gamma^{s}=\frac{1}{2}\left(\gamma^{0}-\gamma^{r}\right), \quad \gamma^{l}=\frac{1}{2}\left(\gamma^{0}+\gamma^{r}\right),
$$

the fermionic projector takes the form

$$
\begin{aligned}
& P(x, y)=\text { (smooth contributions) } \\
& +\sum_{\beta=1}^{3} \frac{1}{i r} \sum_{k=0}^{\infty} \frac{(-i r)^{k}}{k !} \int_{\Xi_{\beta}} d \omega h_{\beta} \alpha_{\beta}^{k} e^{i \omega s} \\
& -\gamma^{s} \sum_{\beta=1}^{3} \frac{1}{i r} \sum_{k=0}^{\infty} \frac{(-i r)^{k}}{k !} \int_{\Xi_{\beta}} d \omega\left(-2 \omega g-f-\frac{k}{k+1} g \alpha_{\beta}\right) \alpha_{\beta}^{k} e^{i \omega s} \\
& +\gamma^{s} \sum_{\beta=1}^{3} \frac{1}{(i r)^{2}} \int_{\Xi_{\beta}} d \omega g e^{i \omega s} \\
& +\gamma^{l} \sum_{\beta=1}^{3} \frac{1}{(i r)^{2}} \sum_{k=0}^{\infty} \frac{(-i r)^{k}}{k !} \int_{\Xi_{\beta}} d \omega\left((k-1) g \alpha_{\beta}^{k}-k f \alpha_{\beta}^{k-1}\right) e^{i \omega s} .
\end{aligned}
$$


The expansion of the scalar component (5.7) is very similar to that in [6, $\S 4.3]$. The only difference is that instead of $l$ we are working here with the "large" coordinate $r$, which is more convenient in the spherically symmetric situation. Setting $r=l+s$ and expanding in powers of $l$, one sees that (5.7) amounts to a special form of the regularization expansion. For the vector component one should notice that the terms $-f-\frac{k}{k+1} g \alpha$ in (5.8) are of higher order in $\alpha_{\max } / E_{P}$ and were thus left out in [6, §4.3]. The term (5.9) can be identified with the summand $n=1$ of the regularization expansion. Comparing (5.10) with the formulas in [6, §4.3], one sees that the function $f$ describes the shear of the surface states. To summarize, the expansion (5.75.10) is compatible with the general expansion in [6], but in our spherical situation the regularization expansion has a special form.

We can now describe our general strategy for constructing admissible regularizations. Without a regularization, the mass expansion corresponds in position space to an expansion in powers of $s$ (as one sees for example by expanding the Bessel functions as in $(3.2,3.3)$ ). Likewise, in the representation of Lemma 5.2 with regularization, the terms for $k=0$ will be much larger on the light cone than the terms for $k=1$, which will in turn be much larger than the terms for $k=2$, et cetera. It is a crucial observation that without a regularization and away from the light cone (2.20, 3.5), the leading term in the mass expansion is cubic, because the contributions $\sim m^{0}, \sim m$ and $\sim m^{2}$ all drop out in (3.4), as a consequence of the special form of the Fourier transform of the Dirac sea configuration. Once we put in a regularization, these lower order terms in the mass expansion will in general no longer drop out, and this leads to the surprising effect that in $\mathcal{M}\left[A_{x y}^{\varepsilon}\right]$ the regularization terms will be typically much larger on the light cone than what is needed for compensating the singularity of (2.20, 3.5). Without this effect, it would be very hard if not impossible to construct admissible regularization. But exploiting this fact, we can proceed as follows. We choose the regularization functions $g_{\beta}, f_{\beta}$ and $h_{\beta}$ such that their Fourier transforms $g_{\beta}(s), f_{\beta}(s)$ and $h_{\beta}(s)$ are supported in the interval $(-\varepsilon, \varepsilon)$, except for small regularization tails which are more spread out and decay polynomially $\sim s^{-\gamma}$ or logarithmically $\sim s^{-\gamma} \log ^{p} s$. We arrange that the regularization terms drop out in $\mathcal{M}\left[A_{x y}^{\varepsilon}\right]$, except for the regularization tails. We then have a lot of freedom to choose the exponents $\gamma$ and the amplitudes of the regularization tails, and this will indeed make it possible to compensate for the singularity of (2.20, 3.5).

Let us specify which class of regularizations we shall consider. It is desirable that the Fourier transform of the regularization functions can be computed explicitly, because we can then work with closed formulas for the regularized fermionic projector. A function which has a particularly simple Fourier transform is the exponential,

$$
\int_{-\infty}^{0} e^{i \omega s} e^{\varepsilon \omega} d \omega=\int_{0}^{\infty} e^{-i \omega s} e^{-\varepsilon \omega} d \omega=-\frac{i}{s-i \varepsilon}=-\frac{i}{s}+\frac{\varepsilon}{s^{2}}+\frac{i \varepsilon^{2}}{s^{3}}+\cdots .
$$

In the special case $\varepsilon=0$ we simply get the Fourier transform of the Heaviside function. Furthermore, the terms involving the regularization decay as desired on the scale $\varepsilon$. However, unfortunately, the leading regularization term decays at a fixed rate $\sim s^{-2}$. We would like to be more flexible; in particular, we would like to arrange that the regularization term decays at the faster rate $\mathcal{O}\left(s^{-2-n}\right)$ for some $n>0$. The key for getting more general decay rates is the observation that the term $\varepsilon / s^{2}$ arises because the exponential $e^{\varepsilon \omega}$ has a non-vanishing derivative at $\omega=0$. This motivates our general method of multiplying the function $e^{\varepsilon \omega}$ by the truncated Taylor series of $e^{-\varepsilon \omega}$. For the resulting product, the first $n$ derivatives vanish at $\omega=0$. Such so-called polynomial smoothing really give 
faster decay of the regularization terms, as the next lemma shows.

\section{Proposition 5.3 (polynomially smoothed exponential regularization)}

For all $\omega \in \mathbb{R}$ and $\varepsilon>0$,

$$
\int_{0}^{\infty} e^{-i \omega s} e^{-\varepsilon \omega}\left(\sum_{l=0}^{n} \frac{(\varepsilon \omega)^{l}}{l !}\right) d \omega=-\frac{i}{s}+\frac{i}{s}\left(\frac{\varepsilon}{\varepsilon+i s}\right)^{n+1} .
$$

Proof. Rewriting the polynomial in $\omega$ with $\varepsilon$-derivatives, we can evaluate the integral,

$$
\begin{aligned}
\int_{0}^{\infty} e^{-i \omega s-\varepsilon \omega}\left(\sum_{l=0}^{n} \frac{(\varepsilon \omega)^{l}}{l !}\right) d \omega & =\sum_{l=0}^{n} \frac{1}{l !}\left(-\varepsilon \frac{d}{d \varepsilon}\right)^{l} \int_{0}^{\infty} e^{-i \omega s-\varepsilon \omega} d \omega \\
=\sum_{l=0}^{n} \frac{1}{l !}\left(-\varepsilon \frac{d}{d \varepsilon}\right)^{l} \frac{1}{\varepsilon+i s} & =\frac{1}{\varepsilon+i s} \sum_{l=0}^{n}\left(\frac{\varepsilon}{\varepsilon+i s}\right)^{l} .
\end{aligned}
$$

Applying the standard formula

$$
1+a+\cdots+a^{n}=\frac{1-a^{n+1}}{1-a}
$$

gives the result.

In the regularization tails, we want to have a power behavior $\sim|s|^{-\alpha}$ or a log-power behavior $\sim \log (s)|s|^{-\alpha}$, where $\alpha$ can be any positive real number. The method is to work with noninteger powers of $\omega$ in the Fourier integral:

Lemma 5.4 For all $\omega \in \mathbb{R}$ and $\alpha, \rho>0$,

$$
\begin{aligned}
\int_{0}^{\infty} e^{-i \omega s-\rho \omega} \omega^{\alpha-1} d \omega & =\Gamma(\alpha) \exp (-\alpha \log (\rho+i s)) \\
\int_{0}^{\infty} e^{-i \omega s-\rho \omega} \omega^{\alpha-1}\left(\log \omega-\frac{\Gamma^{\prime}(\alpha)}{\Gamma(\alpha)}\right) d \omega & =-\log (\rho+i s) \Gamma(\alpha) \exp (-\alpha \log (\rho+i s)) .
\end{aligned}
$$

Proof. It is most convenient to present the integrals as $\lambda$-derivatives of a generating functional $F(\lambda)$,

$$
\int_{0}^{\infty} e^{-i \omega s-\rho \omega} \omega^{\alpha-1} \log ^{p} \omega d \omega=\left.\frac{d^{p}}{d \lambda^{p}} F(\lambda)\right|_{\lambda=0},
$$

which is computed as follows,

$$
\begin{aligned}
F(\lambda) & =\int_{0}^{\infty} e^{-i \omega s-\rho \omega} \omega^{\alpha-1} e^{\lambda \log \omega} d \omega=\int_{0}^{\infty} e^{-i \omega s-\rho \omega} \omega^{\lambda+\alpha-1} d \omega \\
& =(\rho+i s)^{-\lambda-\alpha} \int_{0}^{\infty} u^{\lambda+\alpha-1} e^{-u} d u=\Gamma(\lambda+\alpha)(\rho+i s)^{-\lambda-\alpha} .
\end{aligned}
$$

In the last line we introduced the new integration variable $u=(\rho+i s) \omega$ and rotated the contour around the origin in the complex plane.

Here the parameter $\rho$ gives the length scale at which the regularization tail comes into play. Namely, in the regime $|s| \gg \rho$, we can simplify the above formulas using the expansions

$$
\begin{aligned}
\log (\rho+i s) & =\left(\log |s|+\frac{i \pi}{2} \epsilon(s)\right)(1+\mathcal{O}(\rho / s)) \\
\exp (-i \alpha \log (\rho+i s)) & =|s|^{-\alpha} \exp \left(-\frac{i \pi}{2} \alpha \epsilon(s)\right)(1+\mathcal{O}(\rho / s))
\end{aligned}
$$


(where $\epsilon$ is again the step function), to obtain the desired power and log-power behavior in $s$. However, the Fourier integrals of Lemma 5.4 have two disadvantages. First, in (5.12) we have an error term linear in $\rho / s$; it would be preferable that the error term is of higher order $(\rho / s)^{n}$. Second, the Fourier integrals in Lemma 5.4 are bounded in the limit $s \rightarrow 0$ (by $\rho^{-\alpha}$ and $\rho^{-\alpha} \log \rho$, respectively), but it would be useful that the Fourier integrals even decay for small $|s|$. The following more general regularization functions remedy these disadvantages by a polynomial smoothing and additional overall $\omega$-derivatives.

Definition 5.5 For given integers $p, q \geq 0$ and positive parameters $\rho, \tilde{\rho}, \alpha$ with $\rho<\tilde{\rho}$, we introduce the following functions:

$$
\begin{aligned}
\hat{\mathrm{R}}^{p, q}(\rho, \alpha, \omega) & =\frac{1}{\Gamma(\alpha+p)}\left(\frac{d}{d \omega}\right)^{p}\left[e^{-\rho \omega} \sum_{l=0}^{q} \frac{(\rho \omega)^{l}}{l !} \omega^{\alpha+p-1}\right] \\
\hat{\mathrm{R}} \log ^{p, q}(\rho, \alpha, \omega) & =-\frac{1}{\Gamma(\alpha+p)}\left(\frac{d}{d \omega}\right)^{p}\left[e^{-\rho \omega} \sum_{l=0}^{q} \frac{(\rho \omega)^{l}}{l !} \omega^{\alpha+p-1}\left(\log \omega-\frac{\Gamma^{\prime}(\alpha)}{\Gamma(\alpha)}\right)\right] \\
\hat{\mathrm{R}}^{p, q}(\rho, \tilde{\rho}, \alpha, \omega) & =\hat{\mathrm{R}}^{p, q}(\rho, \alpha, \omega)-\hat{\mathrm{R}}^{p, q}(\tilde{\rho}, \alpha, \omega) \\
\hat{\mathrm{R}} \log ^{p, q}(\rho, \tilde{\rho}, \alpha, \omega) & =\hat{\mathrm{R}} \log ^{p, q}(\rho, \alpha, \omega)-\hat{\mathrm{R}} \log ^{p, q}(\tilde{\rho}, \alpha, \omega)
\end{aligned}
$$

In the next proposition we collect some properties of the Fourier transforms of the functions $\hat{\mathrm{R}}^{p, q}(\rho, \alpha, \omega)$ and $\hat{\mathrm{R}} \log ^{p, q}(\rho, \alpha, \omega)$.

Proposition 5.6 (regularization tails) The two Fourier integrals

$$
\begin{aligned}
& \mathrm{R}^{p, q}(\rho, \alpha, s):=\int_{0}^{\infty} e^{-i \omega s} \hat{\mathrm{R}}^{p, q}(\rho, \alpha, \omega) d \omega \\
& \operatorname{Rlog}^{p, q}(\rho, \alpha, s):=\int_{0}^{\infty} e^{-i \omega s} \hat{\mathrm{R}} \log ^{p, q}(\rho, \alpha, \omega) d \omega
\end{aligned}
$$

in the region $|s| \gg \rho$ have the asymptotic expansions

$$
\begin{aligned}
& \mathrm{R}^{p, q}(\rho, \alpha, s)=|s|^{-\alpha} \exp \left(-\frac{i \pi}{2} \alpha \epsilon(s)\right)\left(1+\mathcal{O}\left((\rho / s)^{q+1}\right)\right) \\
& \mathrm{R}^{p, q}(\rho, \alpha, s)=\left(\log |s|+\frac{i \pi}{2} \epsilon(s)\right)|s|^{-\alpha} \exp \left(-\frac{i \pi}{2} \alpha \epsilon(s)\right)\left(1+\mathcal{O}\left((\rho / s)^{q+1}\right)\right) .
\end{aligned}
$$

Moreover, there are constants $c=c(p, q)$ such that

$$
\left|\mathrm{R}^{p, q}(\rho, \alpha, s)\right| \leq c(p, q) \rho^{-\alpha-p}|s|^{p}, \quad\left|\operatorname{Rlog}^{p, q}(\rho, \alpha, s)\right| \leq c(p, q)|\log \rho| \rho^{-\alpha-p}|s|^{p} .
$$

The functions $\hat{\mathrm{R}}^{p, q}$ and $\hat{\mathrm{R}} \log ^{p, q}$ satisfy for suitable constants $C=C(p, q)$ the bounds

$$
\left|\hat{\mathrm{R}}^{p, q}(\rho, \alpha, \omega)\right| \leq C(p, q) \rho^{-\alpha+1}, \quad\left|\hat{\mathrm{R}} \log ^{p, q}(\rho, \alpha, \omega)\right| \leq C(p, q)|\log \rho| \rho^{-\alpha+1} .
$$

Proof. The estimate (5.20) follows from a simple scaling argument. Integrating by parts, one sees that the differential operator $\left(\partial_{\omega}\right)^{p}$ corresponds in position space to a multiplication by $(i s)^{p}$. Using this fact, we can compute the Fourier transform of every summand in (5.13) and (5.14) using Lemma 5.4. This gives the result.

Thus in the region $|s| \ll \rho$, the regularization functions $R^{p, q}(\rho, \alpha, s)$ can be made arbitrarily small by choosing $p$ sufficiently large. In the region $|s| \gg \rho$, on the other hand, the 


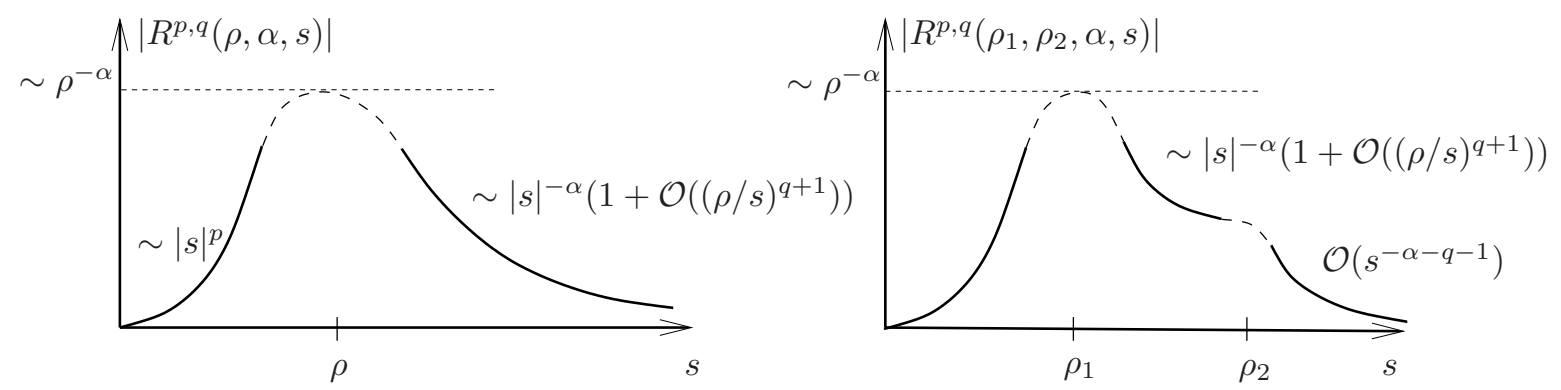

Figure 4: Asymptotics of the functions $\mathrm{R}^{p, q}$.

regularization tail has the desired power behavior $\sim s^{-\alpha}$, up to an error term which can be made arbitrarily small by increasing $q$. This asymptotics is shown on the left of Figure 4. The regularization tails $\mathrm{R}^{p, q}(\rho, \tilde{\rho}, \alpha, s)$ and $\operatorname{Rlog}^{p, q}(\rho, \tilde{\rho}, \alpha, s)$, which involve two length scales $\rho$ and $\tilde{\rho}$, are illustrated on the right of Figure 4 . The second parameter $\tilde{\rho}$ gives an upper scale for the power behavior $\sim|s|^{-\alpha}$ of the tail. If $|s| \gg \tilde{\rho}$, the regularization function can be made arbitrarily small by choosing $q$ large.

Combining the results of Propositions 5.3 and 5.6, we are led to regularization functions of the form

$$
e^{\varepsilon \omega}\left(\sum_{l=0}^{n} \frac{(-\rho \omega)^{l}}{l !}\right)+\kappa_{1} \hat{\mathrm{R}}^{p_{1}, q_{1}}\left(\rho_{1}, \alpha_{1},-\omega\right)+\kappa_{2} \hat{\mathrm{R}} \log ^{p_{2}, q_{2}}\left(\rho_{2}, \alpha_{2},-\omega\right)+\cdots,
$$

where we are still free to adjust the parameters of the regularization tails as functions of $\varepsilon$, and '...' may be a sum of additional regularization tails. It is important to keep in mind that the functions describing the regularization tails should be small in momentum space and should tend to zero as $\varepsilon \searrow 0$. In view of (5.20), this can be ensured by arranging that

$$
\kappa_{i} \rho_{i}^{-\alpha_{i}+1} \log \rho_{i} \ll 1 \text {. }
$$

\section{The Outer Strip}

We now begin the study of $\mathcal{M}\left[A_{x y}^{\varepsilon}\right]$ (see $(2.18,2.15)$ ) for the fermionic projector $P^{\varepsilon}$ with spherically symmetric regularization (see Section 5 ). In order to work out the underlying mechanisms, we begin by discussing the effect of simple contributions to the regularized fermionic projector and proceed by taking into account additional, more complicated contributions. This will lead us to the general construction of Proposition 6.1.

Recall that away from the light cone and without regularization, $\mathcal{M}\left[A_{x y}\right]$ is given by (2.20, 3.4). Naively, one might expect that this formula should to good approximation also be valid for $\mathcal{M}\left[A_{x y}^{\varepsilon}\right]$, except in the strip ||$t|-r| \lesssim \varepsilon$ near the light cone, where the regularization should be important. In order to explain why this naive picture is not correct, we consider for simplicity one Dirac sea and disregard the regularization of the functions $K, f$ and $h$ in (5.1) (i.e. we omit the index $\beta$ and choose $K, f$ and $h$ as in (5.2, 5.3)). For the function $g$ we choose a polynomially smoothed exponential regularization plus a regularization tail,

$$
g(\omega)=e^{\varepsilon \omega}\left(\frac{1}{16 \pi} \sum_{l=0}^{n} \frac{(-\varepsilon \omega)^{l}}{l !}+\frac{\delta}{\Gamma(\gamma)}|\omega|^{\gamma-1}\right) \Theta(-\omega)
$$


with parameters $\gamma>1, n \in \mathbb{N}$ and $\delta>0$. The regularization tail should be small compared to the first term, meaning that

$$
\delta \ll \varepsilon^{\gamma-1} .
$$

Considering the leading terms of the mass expansion of Lemma 5.2 and computing the remaining one-dimensional Fourier transform with the help of Propositions 5.3 and 5.6, we obtain to leading order in $s / r$ the following regularization effect,

$$
\begin{aligned}
P^{\varepsilon}-P & \asymp-\frac{i}{r}\left(\gamma^{0}-\gamma^{r}\right) \int_{-\infty}^{0} \omega\left(g-\frac{1}{16 \pi}\right) e^{i \omega s} d \omega+\frac{1}{r^{2}} \gamma^{r} \int_{-\infty}^{0}\left(g-\frac{1}{16 \pi}\right) e^{i \omega s} d \omega \\
& =\left(-\frac{\gamma^{0}-\gamma^{r}}{r} \frac{\partial}{\partial s}+\frac{\gamma^{r}}{r^{2}}\right)\left[\frac{i}{s}\left(\frac{\varepsilon}{\varepsilon+i s}\right)^{n+1}+\delta e^{-\gamma \log (\varepsilon+i s)}\right]
\end{aligned}
$$

(the symbol " $\asymp$ " means that we pick a particular contribution to $P^{\varepsilon}-P$ ). If $s=\varepsilon$, the condition (6.2) ensures that the contribution by the polynomially smoothed exponential regularization is much larger than the regularization tail,

$$
\left.\left.\frac{i}{s}\left(\frac{\varepsilon}{\varepsilon+i s}\right)^{n+1}\right|_{s=\varepsilon} \sim \frac{1}{\varepsilon} \gg \delta \varepsilon^{-\gamma} \sim \delta e^{-\gamma \log (\varepsilon+i s)}\right|_{s=\varepsilon} .
$$

(Note that differentiating with respect to $s$ changes the scaling only by an overall factor $\varepsilon^{-1}$, and thus the last inequality can also be applied to the derivative term in (6.3).) But, by choosing $n$ large, we can arrange that the contribution by the polynomially smoothed exponential regularization decays faster in $s$ than the regularization tail, and thus the regularization tail dominates if $s \gg \varepsilon$. Furthermore, using the identity (5.12) we obtain the following asymptotic formula for the regularization effect,

$$
\begin{aligned}
P^{\varepsilon}-P \asymp & \left(-\frac{\gamma^{0}-\gamma^{r}}{r} \frac{\partial}{\partial s}+\frac{\gamma^{r}}{r^{2}}\right) \delta|s|^{-\gamma}(\cos (\pi \gamma / 2)-i \epsilon(s) \sin (\pi \gamma / 2)) \\
= & \delta \frac{\gamma^{0}-\gamma^{r}}{r} \gamma|s|^{-\gamma-1}(\epsilon(s) \cos (\pi \gamma / 2)-i \sin (\pi \gamma / 2)) \\
& +\delta \frac{\gamma^{r}}{r^{2}}|s|^{-\gamma}(\cos (\pi \gamma / 2)-i \epsilon(s) \sin (\pi \gamma / 2)) .
\end{aligned}
$$

Let us carefully consider in which range this asymptotic formula is valid. Since we took into account only the leading contribution in $s / r$, we clearly need to assume that $|s| \ll r$. Furthermore, working with the regularization tails is justified only if $s \gg \varepsilon$. Finally, the mass expansion requires that $m^{2} \gg\left|t^{2}-r^{2}\right|=\left|s^{2}+2 s r\right|$ and thus $s \ll m^{-1}, m^{-2} / r$. We conclude that the above asymptotic formula is valid for $s$ in the range

$$
\varepsilon \ll|s| \ll \min \left(m^{-1}, r, m^{-2} / r\right) .
$$

Comparing with the leading contribution to the unregularized fermionic projector,

$$
P \asymp-\frac{i}{16 \pi^{3}}\left(\gamma^{0}-\gamma^{r}\right) \frac{1}{r s^{2}}-\frac{i}{16 \pi^{3}} \gamma^{r} \frac{1}{r^{2} s},
$$

one sees that the regularization terms (6.4, 6.5) are very small. However, as a major difference to the purely imaginary expression (6.7), they also have real components. As a consequence, the regularization terms behave differently in composite expressions. More 
precisely, a direct calculation yields for the traceless part of $A_{x y}^{\varepsilon}$ to leading order in $s / r$ the formula

$$
\begin{aligned}
A_{x y}^{\varepsilon}-\frac{1}{4} \operatorname{Tr}\left(A_{x y}^{\varepsilon}\right) \asymp & \frac{\delta}{8 \pi^{3}} \frac{i \gamma^{0} \gamma^{r}}{r^{3}}(1-\gamma)|s|^{-\gamma-2} \cos (\pi \gamma / 2) \\
& -\frac{m^{3}}{256 \pi^{5}} \frac{\gamma^{0}-\gamma^{r}}{r s^{2}} \Theta(s)-\frac{m^{3}}{256 \pi^{5}} \frac{\gamma^{r}}{r^{2} s} \Theta(s),
\end{aligned}
$$

valid in the range (6.6). We see that the regularization terms give rise to a bilinear contribution to $A_{x y}^{\varepsilon}$. This component involves no powers of $m$, and has thus been "amplified" compared to the vector component, which is $\sim m^{3}$ (see also the discussion on page 26). We remark that there are also contributions by the regularization tail to the vector components of the form $\sim \delta m \gamma^{0}$ and $\sim \delta m \gamma^{r}$; for clarity we postpone their discussion (see (6.32) ).

To compute the corresponding $\mathcal{M}\left[A_{x y}^{\varepsilon}\right]$, we introduce for the decomposition of $A_{x y}^{\varepsilon}$ into its scalar, vector and bilinear components the notation

$$
A_{x y}^{\varepsilon}=A^{s} \mathbb{1}+A^{0} \gamma^{0}-A^{r} \gamma^{r}+A^{b} i \gamma^{0} \gamma^{r} .
$$

Then the roots of the characteristic polynomial of $A_{x y}^{\varepsilon}$ are given by

$$
\lambda_{ \pm}=A^{s} \pm \sqrt{\left(A^{0}\right)^{2}-\left(A^{r}\right)^{2}-\left(A^{b}\right)^{2}} .
$$

Since $A^{s}$ is real, the $\lambda_{ \pm}$form a complex conjugate pair if the discriminant is negative, and in this case the argument given after (2.16) yields that $\mathcal{M}\left[A_{x y}^{\varepsilon}\right]$ vanishes. Using (6.4, 6.5), we conclude that

$$
\mathcal{M}\left[A_{x y}^{\varepsilon}\right]=0 \quad \text { if } s \leq s_{1}:=\left(\frac{32 \pi^{2} \delta}{\sqrt{2} m^{3}}|\cos (\pi \gamma / 2)|\right)^{\frac{2}{2 \gamma+1}} r^{-\frac{3}{2 \gamma+1}} .
$$

We shall never choose $\gamma$ equal to an odd integer, so that the factor $\cos (\pi \gamma / 2)$ will always be nonzero. If conversely $s>s_{1}$, the discriminant is positive, and thus the $\lambda_{ \pm}$are real. Let us verify that they have the same sign. One possible method would be a direct calculation similar as in the proof of Lemma 2.1. It is more elegant to proceed as follows. We can clearly assume that $\lambda_{+} \neq \lambda_{-}$. From (6.9) one sees that $A_{x y}^{\varepsilon}$ commutes with the matrix

$$
\kappa:=\frac{1}{2}\left(\mathbb{1}+\rho \gamma^{0} \gamma^{r}\right)
$$

(where $\rho$ is the pseudoscalar matrix). It is easily verified that $\kappa$ projects on a twodimensional subspace, and that $A_{x y}^{\varepsilon}$ restricted to this subspace is not a multiple of the identity matrix. Hence the characteristic polynomial of the matrix $A_{x y}^{\varepsilon} \mid \operatorname{Im} \kappa: \operatorname{Im} \kappa \rightarrow \operatorname{Im} \kappa$ has precisely the simple roots $\lambda_{+}$and $\lambda_{-}$. Thus

$$
\begin{aligned}
\lambda_{+} \lambda_{-} & =\operatorname{det} A_{x y}^{\varepsilon} \mid \operatorname{Im} \kappa=\operatorname{det}\left(\left.\left.P^{\varepsilon}(x, y)\right|_{P^{\varepsilon}(y, x) \operatorname{Im} \kappa} P^{\varepsilon}(y, x)\right|_{\operatorname{Im} \kappa}\right) \\
& =\operatorname{det}\left(\left.\left(\left.P^{\varepsilon}(y, x)\right|_{\operatorname{Im} \kappa}\right)^{*} P^{\varepsilon}(y, x)\right|_{\operatorname{Im} \kappa}\right)=\left|\operatorname{det}\left(\left.P^{\varepsilon}(y, x)\right|_{\operatorname{Im} \kappa}\right)\right|^{2} \geq 0,
\end{aligned}
$$

and we conclude that $\lambda_{+}$and $\lambda_{-}$indeed have the same sign. Therefore, $\mathcal{M}$ can be computed exactly as explained before (2.18),

$$
\begin{aligned}
& \mathcal{M}\left[A_{x y}^{\varepsilon}\right]=2 A_{x y}^{\varepsilon}-\frac{1}{2} \operatorname{Tr}\left(A_{x y}^{\varepsilon}\right) \quad \text { if } s>s_{1} \\
& \asymp \frac{\delta}{4 \pi^{3}} \frac{i \gamma^{0} \gamma^{r}}{r^{3}}(1-\gamma)|s|^{-\gamma-2} \cos (\pi \gamma / 2)-\frac{m^{3}}{128 \pi^{5}}\left(\frac{\gamma^{0}-\gamma^{r}}{r s^{2}}+\frac{\gamma^{r}}{r^{2} s}\right) \Theta(s) .(
\end{aligned}
$$




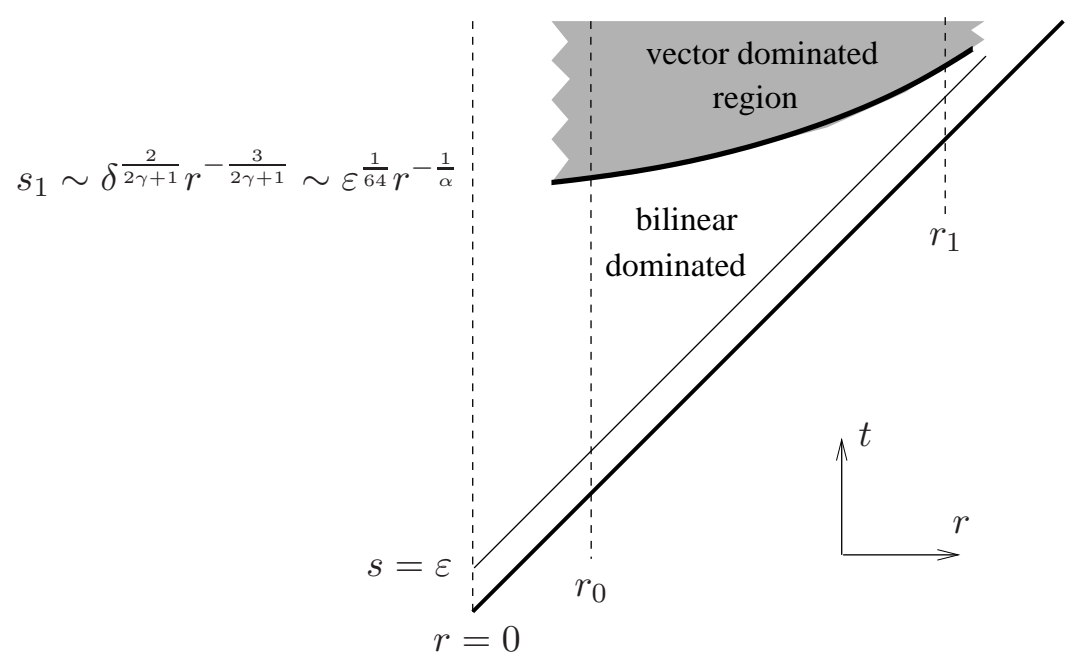

Figure 5: The outer strip.

Let us briefly discuss our results so far. If $s \leq s_{1}$, the bilinear component of $A_{x y}^{\varepsilon}$ makes the discriminant negative. In this so-called bilinear dominated regime, $\mathcal{M}\left[A_{x y}^{\varepsilon}\right]$ vanishes identically. The region $s>s_{1}$, on the other hand, is vector dominated, and $\mathcal{M}\left[A_{x y}^{\varepsilon}\right]$ is nontrivial. The common boundary of these two regions is the surface $s=s_{1}$. This surface is not Lorentz invariant; instead it has a power law scaling, $s_{1} \sim r^{-\frac{3}{2 \gamma+1}}$. Clearly, the above considerations are only valid if the conditions (6.6) and (6.2) are satisfied. This implies in particular that $r$ must lie in an interval $\left(r_{0}, r_{1}\right)$ with

$$
r_{0} \gg \delta^{\frac{1}{\gamma+2}}, \quad r_{1} \ll \varepsilon^{-\frac{2 \gamma+1}{3}} \delta^{\frac{2}{3}} .
$$

The region

$$
r_{0}<r<r_{1}, \quad s_{1}<s \ll r
$$

(and similarly also the region $r_{0}<r<r_{1}, s_{1}<-t-r \ll r$ in the past) is referred to as the outer strip. The outer strip and our scalings are illustrated in Figure 5 (with the parameter $\alpha$ and the power of $\varepsilon$ as they will used in Proposition 6.1 below).

The moments of $\mathcal{M}\left[A_{x y}^{\varepsilon}\right]$ can be introduced similar to (3.9 3.12). However, we are not allowed to take the limit $s_{0} \searrow 0$, because this limit must be performed after taking the limit $\varepsilon \searrow 0$. Furthermore, we need here to take into account the bilinear component, without counterterm. We thus define

$$
\begin{aligned}
I_{\varepsilon}\left(s_{0}, r\right) & =\int_{-s_{0}}^{s_{0}} \mathcal{M}\left[A_{x y}^{\varepsilon}\right] d s+\left(\gamma^{0}-\gamma^{r}\right)\left(\frac{\mathfrak{m}_{3}}{4 r s_{0}}-\frac{\mathfrak{m}_{5}}{2} \log s_{0}\right)-\gamma^{r} \frac{\mathfrak{m}_{3}}{4 r^{2}} \log s_{0} \\
J_{\varepsilon}\left(s_{0}, r\right) & =\int_{-s_{0}}^{s_{0}} s \mathcal{M}\left[A_{x y}^{\varepsilon}\right] d s-\left(\gamma^{0}-\gamma^{r}\right) \frac{\mathfrak{m}_{3}}{4 r} \log s_{0} \\
K_{n, \varepsilon}\left(s_{0}, r\right) & =\int_{-s_{0}}^{s_{0}} s^{n} \mathcal{M}\left[A_{x y}^{\varepsilon}\right] d s, \quad n \geq 2,
\end{aligned}
$$

and denote the vector and bilinear components as in (6.9) by a superscript. Using 6.11, 6.13), we can immediately compute the contribution of the outer strip to the moments. 
For example,

$$
\begin{aligned}
J_{\varepsilon}^{0} & =\int_{s_{1}}^{s_{0}} s \mathcal{M}^{0}\left[A_{x y}^{\varepsilon}\right] d s+\frac{m^{3}}{128 \pi^{5}} \frac{1}{r} \log \left(s_{0}\right) \\
& \asymp-\int_{s_{1}}^{s_{0}} \frac{m^{3}}{128 \pi^{5}} \frac{1}{r s} d s+\frac{m^{3}}{128 \pi^{5}} \frac{1}{r} \log \left(s_{0}\right)=\frac{m^{3}}{128 \pi^{5}} \frac{1}{r} \log \left(s_{1}\right) \\
J_{\varepsilon}^{b} & =\int_{s_{1}}^{s_{0}} s \mathcal{M}^{b}\left[A_{x y}^{\varepsilon}\right] d s \asymp \int_{s_{1}}^{s_{0}} \frac{\delta}{4 \pi^{3}} \frac{1}{r^{3}}(1-\gamma) s^{-\gamma-1} \cos (\pi \gamma / 2) \\
& =\frac{\delta}{4 \pi^{3}} \frac{\gamma-1}{\gamma} \cos (\pi \gamma / 2)\left(s_{0}^{-\gamma}-s_{1}^{-\gamma}\right) .
\end{aligned}
$$

Removing the regularization corresponds to the simultaneous limits $\varepsilon, \delta \searrow 0$, where $\delta=$ $\delta(\varepsilon)$ is to be chosen in agreement with (6.2). We see from (6.15) and (6.11) that $J_{\varepsilon}^{0}$ will diverge in this limit,

$$
J_{\varepsilon}^{0} \sim \log s_{1} \sim \log \delta .
$$

A contribution to a moment which diverges when the regularization is removed is called a singular contribution. The appearance of singular contributions shows that the constructions so far are not yet sufficient; we must find a way to compensate for these divergences. However, before doing so, we discuss the structure of the singular contributions in more detail. The moment $I_{\varepsilon}^{0}$ is also singular and diverges even like a negative power of $\delta$,

$$
I_{\varepsilon}^{0} \sim s_{1}^{-1} \sim \delta^{-\frac{2}{2 \gamma+1}} .
$$

The bilinear component is more regular than the corresponding vector component, but $I^{b}$ is nevertheless singular,

$$
\left|J_{\varepsilon}^{b}\right| \lesssim \delta s_{1}^{-\gamma} \sim \delta^{\frac{1}{2 \gamma+1}}, \quad I_{\varepsilon}^{b} \sim \delta s_{1}^{-1-\gamma} \sim \delta^{-\frac{1}{2 \gamma+1}} .
$$

More generally, the singularities of the moments satisfy the following scaling rules. First, we observe that the vector component in (6.13) is, to leading order in $s / r$, proportional to the nilpotent matrix $\gamma^{0}-\gamma^{r}$. This observation can also be expressed by saying that the vector component of $\mathcal{M}\left[A_{x y}^{\varepsilon}\right]$ is null on the light cone. Since the singular contributions to the moments are determined by the behavior of $\mathcal{M}\left[A_{x y}^{\varepsilon}\right]$ on the light cone, we come to the following simple conclusion.

(R1) The leading singular contributions to the radial and time components of a moment coincide.

The fact that the vector component of $\mathcal{M}\left[A_{x y}^{\varepsilon}\right]$ is null on the light cone also yields a relation between the vector and bilinear components of the moments. Namely, from (6.13) one sees that

$$
\left(\mathcal{M}^{0}\right)^{2}-\left(\mathcal{M}^{r}\right)^{2} \sim \frac{s}{r}\left(\mathcal{M}^{0}\right)^{2}
$$

Since the discriminant in (6.10) vanishes at $s_{1}$,

$$
\left.\frac{s_{1}}{r}\left(\mathcal{M}^{0}\right)^{2}\right|_{s=s_{1}} \sim\left(\mathcal{M}^{0}\right)^{2}-\left.\left(\mathcal{M}^{r}\right)^{2}\right|_{s=s_{1}}=\left.\left(\mathcal{M}^{b}\right)^{2}\right|_{s=s_{1}},
$$

and thus

$$
\mathcal{M}^{b}\left(s_{1}\right) \sim \sqrt{\frac{s_{1}}{r}} \mathcal{M}^{0}\left(s_{1}\right) .
$$

Since $\mathcal{M}$ has a power law behavior in $s$, integrating over s from $s_{1}$ to $s_{0}$ changes the scaling simply by an additional factor $s_{1}$. This explains the following rule. 
(R2) The leading singular contribution to the bilinear component of a moment is smaller than the corresponding vector component by a scaling factor $\left(s_{1} / r\right)^{\frac{1}{2}}$.

Let us now think about how to compensate for the singular contributions to the momenta. One method is to take into account the regularization of the scalar component of $P^{\varepsilon}$. To this end, we choose the function $h$ in (5.1) in analogy to (6.1, 6.2) as

$$
h(\omega)=e^{\varepsilon \omega}\left(\frac{m}{16 \pi} \sum_{l=0}^{n} \frac{(-\varepsilon \omega)^{l}}{l !}+\frac{\kappa m}{\Gamma(\alpha)}|\omega|^{\alpha-1}\right) \Theta(-\omega)
$$

with parameters $\alpha>1$ and

$$
\kappa \ll \varepsilon^{\alpha-1} .
$$

After choosing $n$ sufficiently large, we can again restrict attention to the regularization tail. The leading contribution in $s / r$ of the regularization tail to the fermionic projector is

$$
P^{\varepsilon} \asymp-\kappa \frac{i m}{r}|s|^{-\alpha}(\cos (\pi \alpha / 2)-i \epsilon(s) \sin (\pi \alpha / 2)) .
$$

This gives rise to the following contribution to the traceless part of $A_{x y}^{\varepsilon}$,

$$
A_{x y}^{\varepsilon}-\frac{1}{4} \operatorname{Tr}\left(A_{x y}^{\varepsilon}\right) \asymp \frac{\kappa m}{8 \pi^{3}} \cos (\pi \alpha / 2)\left(\frac{\gamma^{0}-\gamma^{r}}{r^{2}}|s|^{-\alpha-2}+\frac{\gamma^{r}}{r^{3}} \epsilon(s)|s|^{-\alpha-1}\right),
$$

again valid in the range (6.6). Note that we get no bilinear contribution. The vector contribution is of lower order in $m$ than that in (6.8), and thus the regularization tail has again been "amplified" (we shall always choose $\alpha$ such that $\cos (\pi \alpha / 2) \neq 0$ ). The vector component is again null on the light cone.

We next compute the matrix $\mathcal{M}\left[A_{x y}^{\varepsilon}\right]$ corresponding to $(6.8)+(6.21)$. A short calculation shows that, to leading order in $s / r$,

$$
\left(A^{0}\right)^{2}-\left(A^{r}\right)^{2}=\frac{2 s}{r}\left(A^{0}\right)^{2},
$$

and thus we can say that the discriminant is positive if and only if

$$
s>0 \text { and } \sqrt{\frac{2 s}{r}}\left|A^{0}\right| \geq\left|A^{b}\right| .
$$

In particular, for negative $s$ the discriminant is negative and $\mathcal{M}\left[A_{x y}^{\varepsilon}\right]$ vanishes. Hence in what follows we may restrict attention to the region $s>0$. Putting in the detailed formulas and abbreviating the appearing constants by $c_{1}, c_{2}, c_{3}$, we thus obtain

$$
A_{x y}^{\varepsilon} \text { vector dominated } \Longleftrightarrow\left|-c_{1} r^{-\frac{3}{2}} s^{-\frac{3}{2}}+c_{2} \kappa r^{-\frac{5}{2}} s^{-\alpha-\frac{3}{2}}\right| \geq\left|c_{3} \delta\right| r^{-3} s^{-\gamma-2} .
$$

If condition (6.24) is satisfied, we find similar to (6.12) that

$$
\mathcal{M}\left[A_{x y}^{\varepsilon}\right]=2 A_{x y}^{\varepsilon}-\frac{1}{2} \operatorname{Tr}\left(A_{x y}^{\varepsilon}\right) \quad \text { if } A_{x y}^{\varepsilon} \text { vector dominated },
$$

whereas $\mathcal{M}\left[A_{x y}^{\varepsilon}\right]=0$ otherwise. 
The situation described by (6.24, 6.25) is complicated for the following reason. Since we want the new term $\sim \kappa$ to compensate for the singularity of the vector component, we want to choose $\kappa$ positive. Then $A^{0}$ changes sign, having a zero at

$$
s_{2}=\left(\frac{c_{2} \kappa}{c_{1} r}\right)^{\frac{1}{\alpha}} \text {. }
$$

Since the bilinear component of $A$ does not vanish at $s_{2}$, it will dominate the vector component in a neighborhood of $s_{2}$. This gives rise to a so-called intermediate bilinear dominated strip around $s=s_{2}$. The size $\Delta s$ of this strip is of the order $s_{1}$. Thus it is impossible to treat the intermediate bilinear dominated strip with a Taylor expansion in $\Delta s / s_{1}$, making the analytic details rather difficult. In order to avoid this difficulty, it is convenient to take into account the regularization tail of the next term in the mass expansion of the vector component of $P^{\varepsilon}$ by choosing $\alpha(\omega)$ in such a way that

$$
\alpha(\omega) g(\omega)=e^{\varepsilon \omega}\left(\frac{1}{16 \pi}\left(|\omega|-\sqrt{\omega^{2}-k^{2}}\right) \sum_{l=0}^{n} \frac{(-\varepsilon \omega)^{l}}{l !}+\frac{m^{2} \delta_{1}}{\Gamma(\gamma)}|\omega|^{\gamma-\alpha-1} \Theta(-\omega)\right) .
$$

The corresponding contribution to the fermionic projector is

$$
P^{\varepsilon} \asymp-i \delta_{1} m^{2}\left(\gamma^{0}-\gamma^{r}\right)(\gamma-\alpha)|s|^{-\gamma+\alpha-1}(\epsilon(s) \cos (\pi(\gamma-\alpha) / 2)-i \sin (\pi(\gamma-\alpha) / 2)),
$$

and this gives rise to an additional bilinear contribution to $A_{x y}^{\varepsilon}$,

$$
A_{x y}^{\varepsilon}-\frac{1}{4} \operatorname{Tr}\left(A_{x y}^{\varepsilon}\right) \asymp \frac{\delta_{1}}{8 \pi^{3}} \frac{i \gamma^{0} \gamma^{r}}{r^{2}}(\gamma-\alpha)|s|^{-\gamma+\alpha-2} \epsilon(s) \sin (\pi(\gamma-\alpha) / 2) .
$$

By choosing $\delta_{1}$ appropriately, we can arrange that the bilinear component of (6.8) $+(6.27)$ also has a zero at $s_{2},(6.26)$. Thus the vector component dominates also in a neighborhood of $s_{2}$, and the bilinear dominated intermediate strip disappears. Clearly, this method works only if $\gamma>\alpha+1$ and if the resulting $\delta_{1}$ is sufficiently small, but we shall see below that these conditions will automatically be satisfied. Using this method, the bilinear and vector dominated regions look again as in Figure 5 . The left boundary of the vector dominated region is again denoted by $s_{1}$.

The following radial scaling argument gives us a relation between the parameters $\alpha$ and $\gamma$. We want to have cancellations in the vector component when computing a certain moment. Even without specifying in which moment the cancellation should appear, the fact that the cancellation should occur for all values of $r$ allows us to conclude that the graphs in Figure 5 should be independent of $r$ except for scalings of the coordinates. In particular, the quotient $s_{1} / s_{2}$ should be independent of the radius,

$$
\frac{s_{1}}{s_{2}} \sim r^{0} .
$$

As a consequence, using (6.26),

$$
\begin{aligned}
& -c_{1} r^{-\frac{3}{2}} s_{1}^{-\frac{3}{2}}+c_{2} \kappa r^{-\frac{5}{2}} s_{1}^{-\alpha-\frac{3}{2}}=-c_{1} r^{-\frac{3}{2}} s_{2}^{-\frac{3}{2}}\left[\left(\frac{s_{1}}{s_{2}}\right)^{-\frac{3}{2}}-\left(\frac{s_{1}}{s_{2}}\right)^{-\alpha-\frac{3}{2}}\right] \\
& \sim r^{-\frac{3}{2}}\left(r^{-\frac{1}{\alpha}}\right)^{-\frac{3}{2}}=r^{\frac{-3 \alpha+3}{2 \alpha}} \\
& c_{3} \delta r^{-3} s_{1}^{-\gamma-2}=c_{3} \delta r^{-3} s_{2}^{-\gamma-2}\left(\frac{s_{1}}{s_{2}}\right)^{-\gamma-2} \sim r^{-3}\left(r^{-\frac{1}{\alpha}}\right)^{-\gamma-2}=r^{\frac{-3 \alpha+\gamma+2}{\alpha}} .
\end{aligned}
$$


Since at $s_{1}$, (6.24) holds with equality, we conclude that the scalings in (6.29) and (6.30) coincide, and thus

$$
2 \gamma+1=3 \alpha
$$

We next discuss the vector component $\sim \delta$ to $\mathcal{M}\left[A_{x y}^{\varepsilon}\right]$, which was disregarded in (6.8). The contribution (6.4, 6.5) to the fermionic projector gives rise to a corresponding contribution to the vector component of the closed chain,

$$
A_{x y}^{\varepsilon} \asymp-\delta \frac{m \gamma}{8 \pi^{3}}\left(\gamma^{0}-\gamma^{r}\right) \frac{|s|^{-2-\gamma}}{r^{2}} \cos (\pi \gamma / 2)-\delta \frac{m}{8 \pi^{3}} \gamma^{r} \epsilon(s) \frac{|s|^{-1-\gamma}}{r^{3}} \cos (\pi \gamma / 2) .
$$

Using (6.11, 6.2), one sees that at $s_{1},(6.32)$ is smaller than the vector component in (6.8) by a scaling factor $\sqrt{s_{1} r}$. Hence, it gives rise to a contribution to the vector component of the moments, which is smaller than the leading contribution by a scaling factor $\sqrt{s_{1} r}$. Comparing with (6.21), one sees that the contribution $\sim\left(\gamma^{0}-\gamma^{r}\right)$ of (6.32) can be compensated by inserting an additional scalar regularization tail into (6.18) of the form

$$
\frac{\delta \gamma m}{\Gamma(\gamma)}|\omega|^{\gamma-1}
$$

Note that this does not compensate for the contribution $\sim \gamma^{r}$ of (6.32). However, this last term is smaller by an additional scaling factor $s / r$, and as a consequence it will not contribute to any of the moments. Another complication is that (6.32) also contributes to the discriminant. This gives rise to corrections to $s_{1}$, which in turn yield corrections to the moments which are again smaller by a scaling factor $\sqrt{s_{1} r}$ than the leading contributions. Fortunately, all these corrections can be compensated by taking into account additional regularization tails of the scalar component of $P^{\varepsilon}$. The last complication is that these additional regularization tails as well as (6.32) also yield corrections to the zeros of the vector and bilinear components of $A_{x y}^{\varepsilon}$. In particular, these two zeros no longer coincide, giving rise to an intermediate bilinear dominated strip near $s_{2}$. However, the size $\Delta s$ of this strip now scales like $s_{1}^{3 / 2} \sqrt{r}$ and can thus be treated by a simple expansion in powers of $\Delta s / s_{1}$ (for details see the proof of Proposition 6.1 below).

Before coming to the general construction, we point out a structural difference between the vector components in (6.8, 6.21) and (6.32): whereas in (6.8, 6.21) the vector component is a scalar multiple of the matrix

$$
\cdots\left(\left(\gamma^{0}-\gamma^{r}\right)+\frac{s}{r} \gamma^{r}\right)
$$

in (6.32) the radial and time components have a different relative form. This can be understood as follows. As shown before (3.16), a Lorentz invariant contribution is always of the form (6.33). This immediately explains why (6.8) satisfies (6.33). For the term (6.21), which is not Lorentz invariant, we observe that (6.21) comes about as the product of the scalar regularization tail (6.20) with the unregularized vector component of the fermionic projector. The unregularized vector component is clearly of the form (6.33), and this structure is preserved when multiplying by the scalar regularization tail. In the contribution (6.32), however, the vector regularization tail (6.4 6.5) comes into play, which violates (6.33). It is actually very helpful that (6.32) is negligible, so that the vector component of $\mathcal{M}\left[A_{x y}^{\varepsilon}\right]$ satisfies (6.33). Namely, integrating over $s$, we can restate (R1) in the following stronger form: 
(R1') The leading singular contributions to the radial and time components of the moments satisfy the following relations,

$$
I_{\varepsilon}^{r}=I_{\varepsilon}^{0}-\frac{1}{r} J_{\varepsilon}^{0}, \quad J_{\varepsilon}^{r}=J_{\varepsilon}^{0}-\frac{1}{r} K_{2, \varepsilon}^{0}, \quad K_{n, \varepsilon}^{r}=K_{n, \varepsilon}^{0}-\frac{1}{r} K_{n+1, \varepsilon}^{0} .
$$

In view of (3.15), it will be sufficient to consider the time component of the moments; the corresponding relations for the radial component will follow automatically.

We can now state the main result of this section. Anticipating that the construction of Section 7 will allow us to modify $\mathcal{M}\left[A_{x y}^{\varepsilon}\right]$ on an "intermediate layer" $s \approx s_{I}$ with $s_{I} \ll s_{1}$, giving a significant contribution to $I_{\varepsilon}$, but leaving all other moments unchanged, we do not need to consider $I_{\varepsilon}$ here. Thus we want to use the scalar regularization tail 6.20, 6.21) to compensate for the singular contribution to $J_{\varepsilon}^{0}$. According to (6.17), we must compensate for a logarithmic divergence. The logarithm does not cause major problems; the only difference compared to our above discussion is that we must also use the log-power regularization tails $\sim|\omega|^{\alpha-1} \log |\omega|$ as considered in Lemma 5.4. Another modification is that we model the regularization tails more generally with the functions $\hat{\mathrm{R}}^{p, q}$ and $\hat{\mathrm{R}} \log ^{p, q}$ introduced in Definition 5.13. We choose the length scale $\rho$ of the regularization tails equal to $\varepsilon^{\frac{3}{64}} \gg \varepsilon$. Choosing $\rho$ so large makes it is easier to satisfy the condition (5.21). Furthermore, we will choose the parameter $q$ so large that the error terms of order $(\rho / s)^{q}$ will vanish as $\varepsilon \searrow 0$. The parameter $p$, on the other hand, remains undetermined (it will be specified later, see Proposition [7.1).

Proposition 6.1 For any $c_{0} \in \mathbb{R}$ and $\alpha>1$ in the range

$$
1<\alpha<\frac{3}{2}
$$

there is a family of fermionic projectors $\left(P^{\varepsilon}\right)_{\varepsilon>0}$ with spherically symmetric regularization (5.1) having the following properties. The region $r_{0}<r<r_{1}, s_{1}<s \ll r$ with

$$
r_{0} \gg \varepsilon^{\frac{\alpha}{64(\alpha+1)}}, \quad r_{1} \ll \min \left(\varepsilon^{-\frac{\alpha}{32}}, \varepsilon^{-\frac{\alpha}{64(\alpha-1)}}\right), \quad s_{1}(r)=\varepsilon^{\frac{1}{64}} r^{-\frac{1}{\alpha}}
$$

is an outer strip in the sense that

$$
\mathcal{M}\left[A_{x y}^{\varepsilon}\right] \equiv 0 \quad \text { for } \varepsilon \ll s \leq s_{1},
$$

whereas the region $s_{1} \leq s \ll r$ is vector dominated, except for an intermediate bilinear dominated strip of the size $\Delta s \sim s_{1}^{3 / 2} \sqrt{r}$. As $\varepsilon \searrow 0$, the contribution of the outer strip to the moments behaves like

$$
\begin{aligned}
& \lim _{s_{0} \searrow 0} \lim _{\varepsilon \searrow 0}\left\{I_{\varepsilon}^{0}\left(s_{0}, r\right)-r^{-1+\frac{1}{\alpha}} \varepsilon^{-\frac{1}{64}}\left(u_{1}+u_{2} \log r+u_{3} \log \epsilon\right)\right. \\
& -r^{-\frac{1}{2}+\frac{1}{2 \alpha}} \varepsilon^{-\frac{1}{128}}\left(v_{1}+v_{2} \log r+v_{3} \log \epsilon\right) \\
& \left.-r^{-2}\left(w_{1}+w_{2} \log r+w_{3} \log \epsilon\right)\right\}=0 \\
& \lim _{s_{0} \searrow 0} \lim _{\varepsilon \searrow 0}\left\{I_{\varepsilon}^{b}\left(s_{0}, r\right)-r^{-\frac{3}{2}+\frac{1}{2 \alpha}} \varepsilon^{-\frac{1}{128}}\left(b_{1}+b_{2} \log r+b_{3} \log \epsilon\right)\right\}=0 \\
& \lim _{s_{0} \searrow 0 \varepsilon \searrow 0} \lim _{\varepsilon} J_{\varepsilon}^{0}\left(s_{0}, r\right)=\frac{\mathfrak{m}_{3}}{4} \frac{\log 2 r}{r}+\frac{\mathfrak{m}_{3}-2 c_{0}}{8 r} \\
& \lim _{s_{0} \searrow 0} \lim _{\varepsilon \searrow 0} J_{\varepsilon}^{b}\left(s_{0}, r\right)=0 \\
& \lim _{s_{0} \searrow 0} \lim _{\varepsilon \searrow 0} K_{n, \varepsilon}\left(s_{0}, r\right)=0
\end{aligned}
$$


with suitable real parameters $v_{i}, w_{i}$ and $b_{i}$. The radial component of the moments is given by the rule (R1').

Proof. We choose a spherically symmetric regularization (5.1) with $K_{\beta}$ and $\alpha_{\beta}$ according to (5.25.5) and $f_{\beta} \equiv 0$. The regularization functions $g, h$ all vanish for positive $\omega$, whereas for negative $\omega$ they should satisfy the conditions

$$
\begin{aligned}
& \sum_{\beta=1}^{3} h_{\beta}(\omega)=e^{\varepsilon \omega} \frac{M_{1}}{16 \pi} \sum_{l=0}^{n} \frac{(-\varepsilon \omega)^{l}}{l !} \\
& +\kappa M_{1}\left[\hat{\mathrm{R}}^{p_{1}, q_{1}}\left(\varepsilon^{\frac{3}{64}}, \alpha,-\omega\right)+l_{h} \hat{\mathrm{R}} \log ^{p_{1}, q_{1}}\left(\varepsilon^{\frac{3}{64}}, \alpha,-\omega\right)\right] \\
& +\nu_{1} M_{1}\left[\hat{\mathrm{R}}^{p_{1}, q_{1}}\left(\varepsilon^{\frac{3}{64}}, \frac{3 \alpha-1}{2},-\omega\right)+l_{\nu 1} \hat{\mathrm{R}} \log ^{p_{1}, q_{1}}\left(\varepsilon^{\frac{3}{64}}, \frac{3 \alpha-1}{2},-\omega\right)\right] \\
& \sum_{\beta=1}^{3} g_{\beta}(\omega)=e^{\varepsilon \omega} \frac{M_{0}}{16 \pi} \sum_{l=0}^{n} \frac{(-\varepsilon \omega)^{l}}{l !} \\
& +\delta M_{3}\left[\hat{\mathrm{R}}^{p_{1}, q_{1}}\left(\varepsilon^{\frac{3}{64}}, \frac{3 \alpha-1}{2},-\omega\right)+l_{g} \hat{\mathrm{R}} \log ^{p_{1}, q_{1}}\left(\varepsilon^{\frac{3}{64}}, \frac{3 \alpha-1}{2},-\omega\right)\right] \\
& \sum_{\beta=1}^{3} \alpha_{\beta}(\omega) h_{\beta}(\omega)=e^{\varepsilon \omega} \sum_{\beta=1}^{3} \frac{\rho_{\beta}}{16 \pi}\left(|\omega|-\sqrt{|\omega|^{2}-K_{\beta}(\omega)^{2}}\right) \sum_{l=0}^{n} \frac{(-\varepsilon \omega)^{l}}{l !} \\
& +\nu_{2} M_{3}\left[\hat{\mathrm{R}}^{p_{1}, q_{1}}\left(\varepsilon^{\frac{3}{64}}, \frac{\alpha-1}{2},-\omega\right)+l_{\nu 2} \hat{\mathrm{R}} \log ^{p_{1}, q_{1}}\left(\varepsilon^{\frac{3}{64}}, \frac{\alpha-1}{2},-\omega\right)\right] \\
& +\kappa_{1} M_{3}\left[\hat{\mathrm{R}}^{p_{1}, q_{1}}\left(\varepsilon^{\frac{3}{64}}, \alpha-1,-\omega\right)+l_{h 1} \hat{\mathrm{R}} \log ^{p_{1}, q_{1}}\left(\varepsilon^{\frac{3}{64}}, \alpha-1,-\omega\right)\right] \\
& \sum_{\beta=1}^{3} \alpha_{\beta}(\omega) g_{\beta}(\omega)=e^{\varepsilon \omega} \sum_{\beta=1}^{3} \frac{\rho_{\beta}}{16 \pi}\left(|\omega|-\sqrt{|\omega|^{2}-K_{\beta}(\omega)^{2}}\right) \sum_{l=0}^{n} \frac{(-\varepsilon \omega)^{l}}{l !} \\
& +\delta_{1} M_{2} \hat{\mathrm{R}}^{p_{1}, q_{1}}\left(\varepsilon^{\frac{3}{64}}, \frac{\alpha-1}{2},-\omega\right),
\end{aligned}
$$

where we introduced the constants $M_{n}=\sum_{\beta=1}^{3} \rho_{\beta} m_{\beta}^{n}$, and $p_{1}, q_{1}$ are two integers to be determined later. These are four linear equations for the nine unknown functions $h_{\beta}, g_{\beta}$ and $\alpha_{\beta}$. A short consideration shows that the above conditions can all be satisfied. Note that our ansatz involves the 11 free real parameters $\kappa, \delta, \kappa_{1}, \delta_{1}, \nu_{1}, \nu_{2}, l_{h}, l_{g}, l_{h 1}, l_{\nu 1}$ and $l_{\nu 2}$. These parameters are determined by a lengthy, but straightforward calculation. We here give the individual calculation steps.

Let us first consider only the leading contributions to $A_{x y}^{\varepsilon}$. The scalar regularization tail (6.42) leads to a vector component of $A_{x y}^{\varepsilon}$ of the form

$$
\begin{aligned}
A^{0} & =\frac{\kappa C_{1}}{r^{2} s^{2+\alpha}}\left\{1+C_{2} l_{h} \log (s)+C_{3} \kappa^{-1} r s^{\alpha}\right\} \\
A^{r} & =\left(1-\frac{s}{r}\right) A^{0}
\end{aligned}
$$

(this is very similar to (6.8, 6.21), with the only difference that now also logarithms of $s$ appear). The vector regularization tails (6.45, 6.50) give rise to a bilinear component of $A_{x y}^{\varepsilon}$ of the form

$$
A^{b}=\frac{\delta C_{4}}{r^{3} s^{2+\frac{3 \alpha-1}{2}}}\left\{1+C_{5} l_{g} \log (s)+C_{6} \delta_{1} \delta^{-1} r s^{\alpha}\right\}
$$


(this is analogous to the bilinear component in (6.8) and (6.27)). We choose $l_{g}$ and $\delta_{1}$ such that the curly brackets in (6.51) and (6.53) coincide. This ensures that the zeros of the vector and bilinear components coincide, thereby avoiding an intermediate bilinear dominated strip of size $\Delta s \sim s_{1}$ (see the discussion after (6.26)). The parameter $\delta$ is determined by the condition

$$
\sqrt{\frac{2 s_{1}}{r}} A^{0}\left(s_{1}\right)=A^{b}\left(s_{1}\right)
$$

whereas the condition

$$
\int_{s_{1}}^{s_{0}} s A^{0}(s) d s=\frac{\mathfrak{m}_{3}}{4} \frac{\log 2 r}{r}+\frac{\mathfrak{m}_{3}-2 c_{0}}{8 r}+\rho\left(s_{0}\right)+\mathcal{O}\left(s_{1}\right)
$$

fixes $\kappa$ and $l_{h}$ (here $\rho$ is any function which takes care of the boundary values at $s_{0}$ ).

Next we need to take into account different kinds of correction terms. First, by choosing $q_{1}$ sufficiently large, we can make the error terms of the regularization tails as small as we like. Hence we do not need to consider these error terms here. Furthermore, the vector regularization tail (6.45, 6.50) also leads to a contribution to the vector component of $A_{x y}^{\varepsilon}$, which we denote by a subscript $g$,

$$
\begin{aligned}
& A_{g}^{0}=\frac{\delta C_{7}}{r^{2} s^{2+\frac{3 \alpha-1}{2}}}\left\{1+C_{4} l_{g} \log (s)+C_{5} \delta_{1} \delta^{-1} r s^{\alpha}\right\} \\
& A_{g}^{r}=A^{0}+\frac{\delta C_{8}}{r^{3} s^{1+\frac{3 \alpha-1}{2}}}\left\{1+C_{4} l_{g} \log (s)+C_{5} \delta_{1} \delta^{-1} r s^{\alpha}\right\}
\end{aligned}
$$

which at $s_{1}$ is smaller than (6.51) by a scaling factor $\sqrt{s_{1} r}$ (see the discussion after (6.32) ). The contribution $A_{g}$ gives rise to a correction $\Delta s_{1}$ to the left boundary $s_{1}$ of the vector dominated region, which scales like $\Delta s_{1} \sim s_{1}^{3 / 2} r^{1 / 2}$. Both $A_{g}^{t / r}$ and the correction $\Delta s_{1}$ give rise to additional contributions to the vector component of the moments, which are smaller by a scaling factor $\sqrt{s_{1} r}$ than the leading contributions. In order to compensate for these additional contributions, we use the scalar regularization tails (6.43, 6.47). They give rise to an additional contribution to the vector component of $A_{x y}^{\varepsilon}$, which we denote by a subscript $\nu$. This contribution also yields a correction to $s_{1}$ and to the moments. We choose the constants $\nu_{1}, \nu_{2}$ and $l_{\mu 1}, l_{\mu 2}$ such that all the corrections to $s_{1}$ and to the moment $J^{0}$ cancel each other,

$$
\Delta s_{1}=0, \quad \int_{s_{1}}^{\infty} s\left(A_{g}^{0}-A_{\nu}^{0}\right) d s=0 .
$$

For clarity, we point out that it is impossible to compensate for both (6.54) and (6.55) completely by scalar regularization tails, because $A_{\nu}$ is of the form (6.33), whereas $A_{g}$ is not. As a consequence, 6.54, 6.55) give rise to an intermediate bilinear dominated strip near the zero $s_{2}$ of $A^{0}$, whose size $\Delta s$ is of the order $s_{1}^{3 / 2} r^{1 / 2}$. Since the leading contributions of both $A^{t / r}$ and $A^{b}$ vanish at $s_{2}$, the contribution of this intermediate bilinear dominated strip to the moments will be even of the order $s_{1} r$ smaller than the leading contribution (see below).

So far, we only discussed a few selected contributions of the regularization tails to the fermionic projector, and there are indeed many other contributions which all give rise to additional correction terms. Generally speaking, the corrections to $A_{x y}^{\varepsilon}$ can be 
classified as follows. The mass expansion gives rise to powers of $s r$, whereas the socalled regularization expansion gives powers of $s / r$. From power counting one sees that the contributions of higher order in $s$ to the bilinear component of the moments vanish. For the vector component of the moments, we need to take into account only the first orders in the mass and regularization expansion. The first order mass expansion terms can be compensated by the scalar regularization tail (6.48). The first order regularization expansion terms, however, are at most logarithmically divergent and give rise precisely to the terms involving $w_{i}$ in (6.36).

Now all the free parameters have been determined. They scale in $\varepsilon$ as follows,

$$
\begin{aligned}
\kappa & \sim \varepsilon^{\frac{\alpha}{64}}(1+C \log \varepsilon), & \kappa_{1} & \sim \varepsilon^{\frac{\alpha}{64}}(1+C \log \varepsilon) \\
\delta & \sim \varepsilon^{\frac{3 \alpha}{128}}(1+C \log \varepsilon), & \delta_{1} & \sim \frac{\delta}{\kappa} \\
\nu_{1} & \sim \varepsilon^{\frac{3 \alpha}{128}}(1+C \log \varepsilon), & \nu_{2} & \sim \varepsilon^{\frac{\alpha}{128}}(1+C \log \varepsilon),
\end{aligned}
$$

where $C$ stands for a different constant each time. Evaluating the condition (5.21) for all regularization functions gives rise to the condition $\alpha<2$. Likewise, the conditions (6.6) yield the values for $r_{0}$ and $r_{1}$.

Now that all the free parameters have been determined, a straightforward calculation shows that the resulting regularized fermionic projectors $P^{\varepsilon}$ have the desired properties.

We point out that the conditions 6.42 6.50 could not be satisfied if we had only one generation. Furthermore, it is noteworthy that $s_{1} \sim \varepsilon^{\frac{1}{64}} \gg \varepsilon$, and thus the outer region lies away from the strip $s \sim \varepsilon$ where $P(x, y)$ is affected considerably by the regularization. We remark for clarity that the precise power $s_{1} \sim \varepsilon^{\frac{1}{64}}$ introduced in (6.35) was only a matter of convenience. We could just as well have realized any other power law $s_{1} \sim \varepsilon^{\nu}$ with $0<\nu<1$.

It is a natural question whether, by taking into account additional regularization tails, one can compensate for some of the singular contributions in (6.36) or (6.37). Our attempts in this direction were not successful. This is clearly no definite answer, but it gives nevertheless an indication that it should indeed not be possible to compensate for the terms in (6.36, 6.37), for the following general reason. Compensating the vector component (6.36) seems impossible because, using a radial scaling argument, one can compensate only for the singular vector component of $J_{\varepsilon}^{0}$ or of $I_{\varepsilon}^{0}$, but not for both moments at a time. Compensating the bilinear contribution (6.37), on the other hand, would make it necessary to consider a regularization tail of the functions $f_{\beta}$. Again using a radial scaling argument, one finds that the corresponding bilinear contribution does not fall off in $s$ fast enough, so that the outer strip is no longer vector dominated.

\section{$7 \quad$ The Intermediate Layers}

In Proposition 6.1, the region $\varepsilon \ll s \ll s_{1}$ was bilinear dominated, and thus $\mathcal{M}\left[A_{x y}^{\varepsilon}\right]$ was trivial. In this section, we want to introduce additional structures in this region, making it possible to compensate for the singular contributions to the moments in (6.36, 6.37). To explain the basic idea, we again restrict attention to one Dirac sea and consider the 
two regularization tails

$$
g(\omega) \asymp e^{\varepsilon \omega} \frac{\delta}{\Gamma(\gamma)}|\omega|^{\gamma-1} \Theta(-\omega), \quad f(\omega) \asymp e^{\varepsilon \omega} \frac{\nu}{\Gamma(\beta)}|\omega|^{\beta-1} \Theta(-\omega)
$$

(where the parameters $\delta, \gamma$ and $\beta$ are different from those in the previous section). These regularization tails give a large contribution to the bilinear component of $A_{x y}^{\varepsilon}$,

$$
A^{b} \asymp-\frac{\delta}{8 \pi^{3}}(\gamma-1) \cos (\pi \gamma / 2) r^{-3}|s|^{-\gamma-2}-\frac{\nu}{8 \pi^{3}} \sin (\pi \beta / 2) r^{-2}|s|^{-\beta-2} \epsilon(s) .
$$

By making this contribution sufficiently large, we can arrange that the bilinear contribution dominates, so that $\mathcal{M}\left[A_{x y}^{\varepsilon}\right]$ vanishes. In order to avoid for $\mathcal{M}\left[A_{x y}^{\varepsilon}\right]$ to be trivial everywhere, we let $\delta$ and $\nu$ have opposite sign. Then the bilinear component has a positive zero at

$$
s_{2}=\left(-\frac{\sin (\pi \beta / 2)}{(\gamma-1) \cos (\pi \gamma / 2)} \frac{\nu r}{\delta}\right)^{\frac{1}{\beta-\gamma}} .
$$

Then a small strip in a neighborhood of $s_{2}$ will be vector dominated, and thus $\mathcal{M}\left[A_{x y}^{\varepsilon}\right]$ will be nonzero inside this strip. We choose the parameters $\nu, \delta, \beta, \gamma$ in such a way that $\varepsilon \ll s_{2} \ll s_{1}$. Also, the contribution (7.2) at $s \approx s_{2}$ should be much larger than the contributions to $A_{x y}^{\varepsilon}$ considered in the previous section. Conversely, the contribution (17.2) should decay so rapidly in $s$ that it is negligible inside the outer region. In this way, the outer strip and the region $s \approx s_{2}$ can be analyzed independent of each other. We refer to the region $s \approx s_{2}$ as the intermediate layer.

In order to model the vector component of $A_{x y}^{\varepsilon}$ in the intermediate layer, the simplest method is to work similar to (6.18) with a scalar regularization tail. Unfortunately, this leads to the following problem. When working with a scalar regularization tail, the vector component of $A_{x y}^{\varepsilon}$ satisfies the condition (6.22), and the intermediate layer is thus determined by the inequalities (6.23). Setting

$$
a=A^{0}\left(s_{2}\right) \quad \text { and } \quad b=\left(A^{b}\right)^{\prime}\left(s_{2}\right),
$$

the width $\Delta s_{2}$ of the intermediate layer is given in linear approximation by

$$
\Delta s_{2}=2 \sqrt{\frac{2 s_{2}}{r}} \frac{a}{b} .
$$

For this approximation to be justified, we need to assume that $\Delta s_{2} \ll s_{2}$, and thus

$$
\frac{a}{b} \ll \sqrt{s_{2} r} .
$$

The leading contribution of the intermediate layer to the moments is computed to be

$$
\begin{aligned}
I^{0} & \sim a \Delta s_{2} \sim \frac{a^{2}}{b} s_{2}^{\frac{1}{2}} r^{-\frac{1}{2}} \\
I^{b} & \sim\left(A^{b}\right)^{\prime \prime}\left(\Delta s_{2}\right)^{3} \sim \frac{a^{3}}{b^{2}} s_{2}^{\frac{1}{2}} r^{-\frac{3}{2}}
\end{aligned}
$$

where in the last step we used the natural scaling $\left(A^{b}\right)^{\prime \prime}\left(s_{2}\right) \sim b / s_{2}$. Using (7.6), we conclude that

$$
\frac{I^{0}}{I^{b}} \sim \frac{b}{a} r \gg \sqrt{\frac{r}{s_{2}}} .
$$


On the other hand, taking the quotient of the leading singular contributions in Proposition 6.1, which we need to compensate, we see from 66.36, 6.37, 6.35) that

$$
\frac{I^{0}}{I^{b}} \sim \varepsilon^{-\frac{1}{64}} r^{\frac{1}{2}+\frac{1}{2 \alpha}}=\sqrt{\frac{r}{s_{1}}} .
$$

The scalings (7.7) and (7.8) contradict our assumption $s_{2} \ll s_{1}$. We conclude that it is impossible to compensate for the leading singular contributions in (6.36) and (6.37).

One idea for avoiding the above contradiction is to work with a vector component of $A_{x y}^{\varepsilon}$ which violates (6.22). To explain the method, we consider the two regularization tails

$$
\alpha(\omega) g(\omega) \asymp e^{\varepsilon \omega} \frac{\delta_{1} m^{2}}{\Gamma(\sigma)}|\omega|^{\sigma-1} \Theta(-\omega), \quad f(\omega) \asymp e^{\varepsilon \omega} \frac{\tilde{\nu}}{\Gamma(\tilde{\beta})}|\omega|^{\tilde{\beta}-1} \Theta(-\omega) .
$$

They yield contributions to the bilinear component of $A_{x y}^{\varepsilon}$ of very similar form,

$$
A^{b} \asymp \frac{\delta_{1} m^{2}}{8 \pi^{3}} \sigma \sin (\pi \sigma / 2) r^{-2}|s|^{-\sigma-2} \epsilon(s)-\frac{\tilde{\nu}}{8 \pi^{3}} \sin (\pi \tilde{\beta} / 2) r^{-2}|s|^{-\tilde{\beta}-2} \epsilon(s),
$$

and by choosing $\tilde{\beta}=\sigma$ and $\tilde{\nu}=m^{2} \sigma \delta_{1}$, we can arrange for these contributions to cancel each other. However, then the regularization tails still contribute to the vector component of $A_{x y}^{\varepsilon}$,

$$
A^{0} \asymp \frac{\delta_{1} m^{3}}{8 \pi^{3}} \sigma \sin (\pi \sigma / 2) r^{-1}|s|^{-\sigma-2} \epsilon(s), \quad A^{r} \asymp\left\{1-\frac{s}{2 r}\right\} A^{0} .
$$

The important point is that, due to the extra factor $1 / 2$ inside the curly brackets, this vector component is not of the form (6.33), and thus (6.22) is violated. By combining (7.9) with a vector contribution to $A_{x y}^{\varepsilon}$ which comes from a scalar regularization tail and thus satisfies (6.33), we get the freedom to adjust the time and radial component of $A_{x y}^{\varepsilon}$ independently, without influence on the bilinear component of $A_{x y}^{\varepsilon}$. This additional freedom can actually be used to modify the scaling in (7.7), thus resolving the above contradiction. Nevertheless, the method does not allow us to compensate for all the moments in (6.36) and (6.37), as the following argument shows. Suppose that $A^{0}, A^{r}$ and $A^{b}$ can be chosen independently. Setting

$$
a=A^{0}\left(s_{2}\right), \quad b=\left(A^{b}\right)^{\prime}\left(s_{2}\right) \quad \text { and } \quad \nu=\left(A^{0}-A^{r}\right)\left(s_{2}\right),
$$

we can correct the scaling in (7.7) by choosing

$$
\nu \gg a \frac{s_{2}}{r},
$$

because then

$$
\begin{aligned}
\Delta s_{2} & \sim \frac{\sqrt{a \nu}}{b}, \quad I^{0} \sim b \Delta s_{2} \\
I^{b} & \sim \frac{b}{s_{2}}\left(\Delta s_{2}\right)^{3} \stackrel{7.10}{=} \frac{\Delta s_{2}}{s_{2}} \sqrt{I^{0}} \sqrt{\nu \Delta s_{2}} .
\end{aligned}
$$

By choosing $a, b$ and $\nu$ appropriately, we can indeed compensate for the leading singularities in (6.36) and (6.37). However, if this is done, (7.11) implies that

$$
\varepsilon^{-\frac{1}{64}} \sim \frac{\Delta s_{2}}{s_{2}} \varepsilon^{-\frac{1}{64}} \sqrt{\nu \Delta s_{2}},
$$




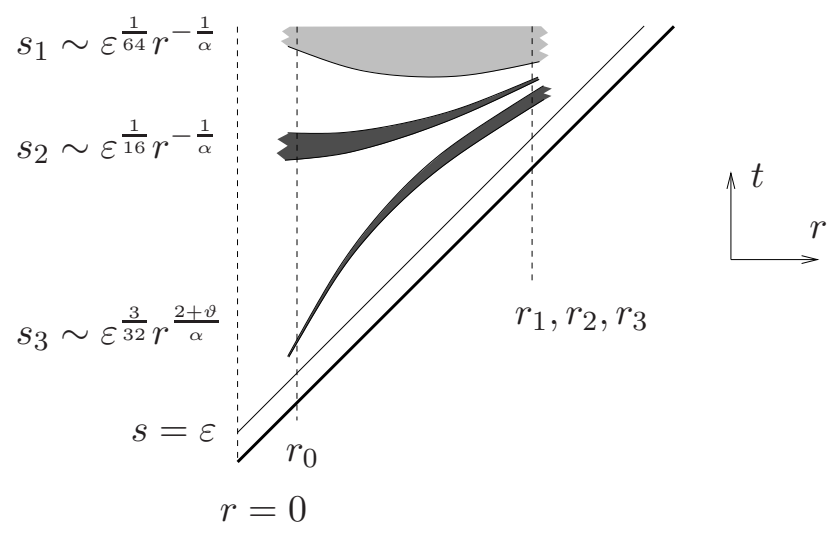

Figure 6: The intermediate layers.

where for simplicity we omitted the scaling in $r$. Clearly, $\Delta s_{2}$ should be much smaller than $s_{2}$, because otherwise we do not have a "strip." We conclude that

$$
\nu \Delta s_{2} \gg 1
$$

Note that, by definition of $\nu$,

$$
I^{r}=I^{0}-\nu \Delta s_{2} .
$$

Hence $I^{r}$ has a nonzero contribution which violates the rule (R1'). Unfortunately, it seems impossible to compensate for this additional contribution to $I^{r}$. This leads us to conclude that working with a vector component of $A_{x y}^{\varepsilon}$ which violates (6.22) does not resolve our problem.

The above consideration explains why it seems difficult to compensate for the leading singular contributions in (6.36) and (6.37) using one intermediate layer. Our way out is to work with two intermediate layers, see Figure 6.

Proposition 7.1 We choose parameters $\vartheta$ and $\tau$ in the range

$$
\alpha<\vartheta<2, \quad 3<\tau<\frac{15}{4} .
$$

Then for any $c_{0}, c_{1} \in \mathbb{R}$, there is a family of fermionic projectors $\left(P^{\varepsilon}\right)_{\varepsilon>0}$ which satisfies the conditions of Proposition 6.1 and moreover has the following properties. Choosing the parameters

$$
r_{0} \gg \varepsilon^{\frac{\alpha}{64(2+\vartheta)}}, \quad r_{2} \ll \varepsilon^{-\frac{\alpha}{64}}, \quad r_{3} \ll \varepsilon^{-\frac{\alpha}{64(2+\vartheta)}}
$$

and setting

$$
\begin{aligned}
s_{2} & =r^{-\frac{1}{\alpha}} \varepsilon^{\frac{1}{16}}, & \Delta s_{2} & =r^{-\frac{2-\alpha+\vartheta}{2 \alpha}} \varepsilon^{\frac{5}{64}} \\
s_{3} & =r^{\frac{2+\vartheta}{\alpha}} \varepsilon^{\frac{3}{32}}, & \Delta s_{3} & =r^{\frac{1}{2}+\frac{(2+\vartheta)(5+2 \tau)}{2 \alpha}} \varepsilon^{\frac{1}{8}},
\end{aligned}
$$

the two layers

$$
\begin{array}{ll}
r_{0}<r<r_{2}, & \left|s-s_{2}\right|<\frac{\Delta s_{2}}{2} \\
r_{0}<r<r_{3}, & \left|s-s_{3}\right|<\frac{\Delta s_{3}}{2}
\end{array}
$$


are intermediate layers in the sense that they are vector dominated, whereas the regions outside these layers and in the range $\varepsilon \ll s<s_{1}$ are bilinear dominated. The contributions of the intermediate layers to the moments satisfy the rule (R1'). Furthermore, they combine with (6.36) and (6.37) in such a way that all singular contributions cancel. The total contributions of the outer strip and the intermediate layers to the moments $I_{\varepsilon}^{0}$ and $I_{\varepsilon}^{b}$ behave like

$$
\begin{aligned}
\lim _{s_{0} \searrow 0} \lim _{\varepsilon \searrow 0} I_{\varepsilon}^{0}\left(s_{0}, t\right) & =\frac{\mathfrak{m}_{3}}{8} \frac{1}{r^{2}}+\frac{\mathfrak{m}_{5}}{2} \log 2 r+\frac{\mathfrak{m}_{3}+c_{1}}{2} \\
\lim _{s_{0} \searrow 0} \lim _{\varepsilon \searrow 0} I_{\varepsilon}^{b}\left(s_{0}, t\right) & =0 .
\end{aligned}
$$

The contribution of the intermediate layers to the moments $J_{\varepsilon}^{b}$ and $J_{\varepsilon}^{0}$ vanishes in the limit $\varepsilon \searrow 0$.

Proof. We choose the regularization functions as in the proof of Proposition 6.1, but add regularization tails as follows. In order to construct the intermediate layer at $s_{2}$, we consider the tails

$$
\begin{aligned}
& \sum_{\beta=1}^{3} h_{\beta}(\omega) \asymp \kappa_{2}\left[\hat{\mathrm{R}}^{p_{2}, q_{2}}\left(\varepsilon^{\frac{9}{128}}, \varepsilon^{\frac{3}{64}}, \vartheta,-\omega\right)+l_{h 2} \hat{\mathrm{R}} \log ^{p_{2}, q_{2}}\left(\varepsilon^{\frac{9}{128}}, \varepsilon^{\frac{3}{64}}, \vartheta,-\omega\right)\right] \\
& \sum_{\beta=1}^{3} g_{\beta}(\omega) \asymp \delta_{2}\left[\hat{\mathrm{R}}^{p_{2}, q_{2}}\left(\varepsilon^{\frac{5}{64}}, \frac{3 \vartheta-1}{2},-\omega\right)+l_{g 2} \hat{\mathrm{R}} \log ^{p_{2}, q_{2}}\left(\varepsilon^{\frac{5}{64}}, \frac{3 \vartheta-1}{2},-\omega\right)\right] \\
& \sum_{\beta=1}^{3} f_{\beta}(\omega) \asymp \nu_{2}\left[\hat{\mathrm{R}}^{p_{2}, q_{2}}\left(\varepsilon^{\frac{5}{64}}, \frac{3 \vartheta-1}{2}-\alpha,-\omega\right)+l_{f 2} \hat{\mathrm{R}} \log ^{p_{2}, q_{2}}\left(\varepsilon^{\frac{5}{64}}, \frac{3 \vartheta-1}{2}-\alpha,-\omega\right)\right]
\end{aligned}
$$

with integers $p_{2}, q_{2}$ and real parameters $\kappa_{2}, \delta_{2}, \nu_{2}$ and $l_{f 2}, l_{g 2}, l_{h 2}$. Note that in (7.15) we use tails involving two length scales (5.15, 5.16). For clarity, we postpone the discussion of the length scales in the regulation tails to Sections 9 and 10. Here it suffices to keep in mind that the tail is "active" in the considered region of $s$, meaning that $\varepsilon^{\frac{5}{64}} \ll|s| \ll \varepsilon^{\frac{3}{64}}$.

Similar to (7.2), the tails (7.16) and (7.17) yield a bilinear contribution to $A_{x y}^{\varepsilon}$. We choose $\delta_{2}$ and $l_{g 2}$ such that this bilinear contribution vanishes at $s_{2}$, (7.13). We set $b=\left(A^{b}\right)^{\prime}\left(s_{2}\right)$. The scalar tail (7.15) gives rise to a vector contribution to $A_{x y}^{\varepsilon}$ which satisfies (R2'). Setting $a=\left(A^{0}\right)^{\prime}\left(s_{2}\right)$, the width $\Delta s_{2}$ of the layer is in linear approximation given by (17.5). We choose $\nu_{2}$ and $l_{f 2}$ such that the resulting value for $\Delta s_{2}$ agrees with (7.13). Using Taylor expansions near $s=s_{2}$, the contribution of the intermediate layer to the bilinear moment is computed to be

$$
I_{\varepsilon}^{b} \asymp-\frac{1}{12}\left(A^{b}\right)^{\prime \prime}\left(s_{2}\right)\left(\Delta s_{2}\right)^{3}+\frac{1}{2}\left(A^{0} \sqrt{\frac{2 s}{r}}\right)^{\prime}\left(s_{2}\right)\left(\Delta s_{2}\right)^{2} .
$$

We choose $\kappa_{2}$ and $l_{h 2}$ such that this contribution compensates for the singular contribution in (6.37). In agreement with our scalings (7.7), the intermediate layer at $s_{2}$ gives rise to a singular contribution to the vector component of the form

$$
I^{0}=\varepsilon^{-\frac{7}{64}} r^{\frac{2-3 \alpha+\vartheta}{2 \alpha}}\left(c_{1}+c_{2} \log \varepsilon+c_{3} \log r\right) .
$$


The just-determined parameters have the following scaling in $\varepsilon$,

$$
\begin{aligned}
\kappa_{2} & \sim \varepsilon^{-\frac{1}{128}+\frac{\vartheta}{16}}(1+C \log \varepsilon), \quad \delta_{2} \sim \varepsilon^{-\frac{3}{128}+\frac{3 \vartheta}{32}}(1+C \log \varepsilon) \\
\nu_{2} & \sim \varepsilon^{-\frac{3}{128}-\frac{\alpha}{16}+\frac{3 \vartheta}{32}}(1+C \log \varepsilon) .
\end{aligned}
$$

A short calculation shows that, using the upper bound for $\vartheta$ in (7.12), the condition (5.21) is satisfied for all regularization tails. Furthermore, choosing $p_{1}>2$, the regularization tails considered in Proposition 6.1 are very small near $s=s_{2}$ and give rise to corrections which all vanish as $\varepsilon \searrow 0$. Likewise, using the lower bound for $\vartheta$ in (7.12), the tails (7.15) 7.17) are so small in the region $s>s_{1}$ that the corresponding error terms in the outer strip vanish in the limit $\varepsilon \searrow 0$. Finally, by choosing $q_{2}$ sufficiently large, we can arrange that the error terms of the tails give no contribution to the moments in the layer at $s_{2}$.

The next step is to construct the intermediate layer near $s_{3}$ in such a way that its contribution to $A^{0}$ compensates for (17.18), whereas its contribution to $A^{b}$ vanishes as $\varepsilon \rightarrow$ 0 . To this end, we introduce the additional regularization tails

$$
\begin{aligned}
& \sum_{\beta=1}^{3} h_{\beta}(\omega) \asymp \kappa_{3}\left[\hat{\mathrm{R}}^{p_{3}, q_{3}}\left(\varepsilon^{\frac{7}{64}}, \varepsilon^{\frac{11}{128}}, \tau,-\omega\right)+l_{h 3} \hat{\mathrm{R}} \log ^{p_{3}, q_{3}}\left(\varepsilon^{\frac{7}{64}}, \varepsilon^{\frac{11}{128}}, \tau,-\omega\right)\right] \\
& \sum_{\beta=1}^{3} g_{\beta}(\omega) \asymp \delta_{3}\left[\hat{\mathrm{R}}^{p_{3}, q_{3}}\left(\varepsilon^{\frac{7}{64}}, 2 \tau+1,-\omega\right)+l_{g 3} \hat{\mathrm{R}} \log ^{p_{3}, q_{3}}\left(\varepsilon^{\frac{7}{64}}, 2 \tau+1,-\omega\right)\right] \\
& \sum_{\beta=1}^{3} f_{\beta}(\omega) \asymp \nu_{3}\left[\hat{\mathrm{R}}^{p_{3}, q_{3}}\left(\varepsilon^{\frac{7}{64}}, 1+2 \tau+\frac{\alpha}{2+\vartheta},-\omega\right)\right. \\
&\left.+l_{f 3} \hat{\mathrm{R}} \log ^{p_{3}, q_{3}}\left(\varepsilon^{\frac{7}{64}}, 1+2 \tau+\frac{\alpha}{2+\vartheta},-\omega\right)\right] .
\end{aligned}
$$

With a similar calculation as in the layer near $s_{2}$ above, we determine the parameters $\delta_{3}, l_{g 3}$ and $\nu_{3}, l_{f 3}$ such as to comply with (7.14). We choose $\kappa_{3}$ and $l_{3}$ such that the contribution of the layer near $s_{3}$ to $I^{0}$ compensates for (7.18). The just-determined parameters have the following scaling in $\varepsilon$,

$$
\begin{aligned}
& \kappa_{3} \sim \varepsilon^{\frac{1}{128}+\frac{3 \tau}{32}}(1+C \log \varepsilon), \quad \delta_{3} \sim \varepsilon^{\frac{15}{128}+\frac{3 \tau}{16}}(1+C \log \varepsilon) \\
& \nu_{3} \sim \varepsilon^{\frac{15}{128}+\frac{\alpha}{64+32 \vartheta}+\frac{3 \tau}{16}(1+C \log \varepsilon),}
\end{aligned}
$$

where $C$ again stands for a different constant each time. A short calculation shows that the upper bound for $\tau$ in (7.12) ensure that the condition (5.21) is satisfied for all regularization tails. Furthermore, choosing $p_{1}$ and $p_{2}$ sufficiently large, the contributions of the tails (7.15 7.17) as well as the tails in Proposition 6.1 to the moments in the layer near $s_{3}$ all vanish in the limit $\varepsilon \searrow 0$. Likewise, using the lower bound for $\tau$ in (7.12), the tails (7.19 7.21) do not contribute to the moments in the layer near $s_{2}$ nor in the outer strip. Moreover, it is straightforward to check that the resulting contribution to $I^{b}$ vanishes as $\varepsilon \searrow 0$. Finally, by choosing $p_{2}$ and $q_{3}$ sufficiently large, we can arrange that the tails (7.15 7.17) as well as the error terms of the tails (7.19 7.21) do not contribute to the moments in the layer at $s_{2}$.

Next we arrange with suitable scalar regularization tails that $I^{0}$ has the desired value. To this end, we consider the scalar regularization tails

$$
\sum_{\beta=1}^{3} h_{\beta}(\omega) \asymp \kappa_{3}\left[\hat{\mathrm{R}}^{0,0}\left(\varepsilon^{\frac{1}{8}}, \tau-\frac{\alpha-\vartheta}{4+2 \vartheta},-\omega\right)+l_{h 3} \hat{\mathrm{R}} \log ^{0,0}\left(\varepsilon^{\frac{1}{8}}, \tau-\frac{\alpha-\vartheta}{4+2 \vartheta},-\omega\right)\right]
$$




$$
\begin{aligned}
& +\kappa_{4}\left[\hat{\mathrm{R}}^{0,0}\left(\varepsilon^{\frac{1}{8}}, \tau-\frac{2 \alpha-\vartheta-1}{4+2 \vartheta},-\omega\right)+l_{h 4} \hat{\mathrm{R}} \log ^{0,0}\left(\varepsilon^{\frac{1}{8}}, \tau-\frac{2 \alpha-\vartheta-1}{4+2 \vartheta},-\omega\right)\right] \\
& +\kappa_{5}\left[\hat{\mathrm{R}}^{0,0}\left(\varepsilon^{\frac{1}{8}}, \tau+\frac{2+\alpha+\vartheta}{4+2 \vartheta},-\omega\right)+l_{h 5} \hat{\mathrm{R}} \log ^{0,0}\left(\varepsilon^{\frac{1}{8}}, \tau+\frac{2+\alpha+\vartheta}{4+2 \vartheta},-\omega\right)\right](7
\end{aligned}
$$

and choose the parameters $\kappa_{i}, l_{h i}$ such that the corresponding contribution in the intermediate layer near $s_{3}$ compensates for all the terms in (6.36) as well as the first summand on the right of (3.13). The second and third summands in (3.13) can be constructed similarly using the regularization tail

$$
\begin{aligned}
\sum_{\beta=1}^{3} h_{\beta}(\omega) \asymp \kappa_{6}[ & \hat{\mathrm{R}}^{0,0}\left(\varepsilon^{\frac{1}{8}}, \tau+\frac{1}{2}-\frac{3 \alpha}{4+2 \vartheta},-\omega\right) \\
& \left.+l_{h 6} \hat{\mathrm{R}} \log ^{0,0}\left(\varepsilon^{\frac{1}{8}}, \tau+\frac{1}{2}-\frac{3 \alpha}{4+2 \vartheta},-\omega\right)\right] .
\end{aligned}
$$

A short calculation shows that the intermediate layers do not contribute to the higher moments $J$ or $K_{n}$. Thus it remains to consider the error terms. More precisely, we need to compensate for the vector component of the moment when we expand in powers of $\Delta s_{2} / s_{2}$ and $\Delta s_{3} / s_{3}$. Moreover, the vector regularization tails give contributions to the vector component of $A_{x y}^{\varepsilon}$, which in analogy to the term (6.32) in the outer strip, are by a factor $\sqrt{s r}$ smaller than the leading terms. Further error terms arise in an expansion in powers of $s / r$. All the resulting contributions to the moments can be compensated in a straightforward way by additional regularization tails.

In order to specify the range of $r$ for which the above arguments hold, one evaluates the conditions (6.6) for all appearing regularization tails.

Finally, we need to verify that the region $-s_{1}<s \ll-\varepsilon$ is everywhere bilinear dominated. Since we used the scalar tails to arrange a nonzero vector component of $A$, this vector component is of the form (6.33). As a consequence, $\left(A^{0}\right)^{2}-\left(A^{r}\right)^{2}<0$, implying that the region is indeed bilinear dominated.

\section{The Innermost Layer}

With the constructions of the previous Sections 6 and 7 we arranged that $\mathcal{M}\left[A_{x y}^{\varepsilon}\right]$ converges on the light cone $t=r>0$ in the distributional sense to $\tilde{\mathcal{M}}(\xi)$. However, so far we have not considered the momentum cone conditions (see Definitions 4.2 and 4.3). In this section, we shall satisfy these additional conditions by introducing another layer, the so-called innermost layer, which lies even closer to the light cone than the intermediate layers. Sometimes we also refer to the intermediate layers together with the innermost layer as the inner layers. We will also have a closer look at the momentum cone conditions, and we will slightly modify them in order to take into account the regularization of $P$ and the fact that $P$ is supported on hyperbolas instead of a cone (compare Figures 1 and 3 ).

In preparation, we consider the matrix structure of the contributions to the regularized product $\mathcal{M}\left[A_{x y}\right] P^{\varepsilon}(x, y)$. We can clearly restrict attention to a vector $\xi$ for which $\mathcal{M}\left[A_{x y}\right]$ is nonzero. From general properties of the characteristic polynomial (see [․, Lemma 5.2.1]) we know that

$$
Q^{\varepsilon}(x, y)=\frac{1}{2} \mathcal{M}\left[A_{x y}^{\varepsilon}\right] P^{\varepsilon}(x, y)=\frac{1}{2} P^{\varepsilon}(x, y) \mathcal{M}\left[A_{y x}^{\varepsilon}\right]
$$


Furthermore, from (2.18) we see that $\mathcal{M}\left[A_{x y}^{\varepsilon}\right]$ equals twice the trace-free part of $A_{x y}$,

$$
\mathcal{M}\left[A_{x y}^{\varepsilon}\right]=2\left(A_{x y}^{0} \gamma^{0}-A_{x y}^{r} \gamma^{r}+A_{x y}^{b} i \gamma^{0} \gamma^{r}\right)
$$

Let us study the symmetry under the transformation $\xi \rightarrow-\xi$. The bilinear component of $A_{x y}$ arises because vector contributions to $P(x, y)$ and $P(y, x)$ anticommute,

$$
A_{x y}^{b} i \gamma^{0} \gamma^{r}=\frac{1}{2}[P(x, y), P(y, x)]
$$

and thus, using that exchanging $x$ and $y$ also flips the sign of $\gamma^{r}$,

$$
A_{x y}^{b}=A_{y x}^{b} .
$$

The vector component of $A_{x y}$, on the other hand, arises if the vector component of $P(x, y)$ is multiplied by the scalar component of $P(y, x)$, or vice versa. In both cases, the corresponding contributions to $P(x, y)$ and $P(y, x)$ commute, and thus

$$
A_{x y}^{0}=A_{y x}^{0} \quad \text { and } \quad A_{x y}^{r}=-A_{y x}^{r} .
$$

Combining the symmetry relations (8.1) and (8.2, 8.3), we can compute $Q^{\varepsilon}(x, y)$,

$$
\begin{aligned}
Q^{\varepsilon}(x, y) & =\frac{1}{2}\left[A_{x y}^{b} i \gamma^{0} \gamma^{r}, P(x, y)\right]+\frac{1}{2}\left\{A_{x y}^{0} \gamma^{0}-A_{x y}^{r} \gamma^{r}, P(x, y)\right\} \\
& =i A^{b}\left(P^{r} \gamma^{0}-P^{0} \gamma^{r}\right)+\left(A^{0} \gamma^{0}-A^{r} \gamma^{r}\right) P^{s}+A^{0} P^{0}-A^{r} P^{r}
\end{aligned}
$$

where in the last line for convenience we omitted the subscript arguments $x$ and $y$. It is remarkable that $Q^{\varepsilon}$ has no bilinear component.

Clearly, the difficulty in evaluating the momentum cone conditions is to handle the singularity on the light cone. Let us first bring the leading singularity into a more convenient form. We restrict attention to the future light cone $t \approx r$. For the scalar condition, we again choose the variables $r$ and $s=t-r$ and expand in powers of $s / r$,

$$
\int_{0}^{\infty} \frac{\mathcal{M}\left[A_{x y}^{\varepsilon}\right]}{t^{2}-r^{2}} d t=\int_{0}^{\infty} \frac{\mathcal{M}\left[A_{x y}^{\varepsilon}\right]}{(2 r+s) s} d t=\int_{0}^{\infty} \frac{\mathcal{M}\left[A_{x y}^{\varepsilon}\right]}{2 r s} d t+\text { (distributional) } .
$$

The vector condition is more difficult to handle because of the radial derivative. Since the derivative $\partial_{t}+\partial_{r}$ tangential to the light cone is less singular than a transversal derivative, it is useful to rewrite the integral in (4.17) as

$$
\begin{aligned}
\int_{0}^{\infty} & \frac{\partial}{\partial r}\left(r \mathcal{M}\left[A_{x y}^{\varepsilon}\right] i \not\right) \frac{t d t}{t^{2}-r^{2}} \\
= & \left.-\int_{0}^{\infty} \frac{\partial}{\partial t}\left(r \mathcal{M}\left[A_{x y}^{\varepsilon}\right] i \not\right)\right) \frac{t d t}{t^{2}-r^{2}}+\int_{0}^{\infty}\left(\partial_{t}+\partial_{r}\right)\left(r \mathcal{M}\left[A_{x y}^{\varepsilon}\right] i \not\right) \frac{t d t}{t^{2}-r^{2}} \\
= & \left.-\int_{0}^{\infty}\left(\mathcal{M}\left[A_{x y}^{\varepsilon}\right] i \not\right) \frac{r\left(t^{2}+r^{2}\right)}{\left(t^{2}-r^{2}\right)^{2}} d t+\int_{0}^{\infty}\left(\partial_{t}+\partial_{r}\right)\left(r \mathcal{M}\left[A_{x y}^{\varepsilon}\right] i \not\right)\right) \frac{t d t}{t^{2}-r^{2}}
\end{aligned}
$$

where in the last line we integrated by parts. In the first integral we can again expand in powers of $s / r$ to obtain

$$
\left.\int_{0}^{\infty} \frac{\partial}{\partial r}\left(r \mathcal{M}\left[A_{x y}^{\varepsilon}\right] i \not\right)\right) \frac{t d t}{t^{2}-r^{2}}=-\frac{r}{2} \int_{0}^{\infty} \frac{\mathcal{M}\left[A_{x y}^{\varepsilon}\right] i \not ̈}{s^{2}} d t+\text { (less singular terms) } .
$$


Let us evaluate the momentum cone conditions for the leading singular terms (8.5) and (8.7) (all the correction terms will be treated in the proof of Proposition 8.1 below). Since $Q^{\varepsilon}$ has no bilinear component (8.4), we may simply disregard the bilinear contributions to (4.15) and (4.17). Thus it suffices to consider the scalar and vector components. As $\mathcal{M}$ has no scalar component, in (4.15) we get only a vector contribution. Combining the symmetry property (8.3) with (8.5), we obtain for the leading singularities the condition

$$
\int_{0}^{\infty} \frac{\mathcal{M}^{0}}{s} d t \asymp\left(\frac{c_{0}}{r}+c_{2} r+\cdots\right)\left(1+o\left(\varepsilon^{0}\right)\right)
$$

(here $o\left(\varepsilon^{0}\right)$ stands for terms which vanish as $\varepsilon \searrow 0$ ). Considering again regularizations with the general property (R2), the corresponding condition for the radial component is automatically satisfied. Similarly, we can compute the scalar contribution to (4.17). Again restricting attention to regularizations where the leading vector contribution satisfies (6.33), combining (8.3) with (8.7) gives

$$
\int_{0}^{\infty} \frac{\mathcal{M}^{0}}{s} d t \asymp\left(c_{1} r+c_{3} r^{3}+\cdots\right)\left(1+o\left(\varepsilon^{0}\right)\right)
$$

and this condition is consistent with and stronger than (8.8). Finally, the bilinear component of $\mathcal{M}$ gives rise to a vector contribution to (8.7). Using the symmetry property (8.2), we get the condition

$$
\int_{0}^{\infty} \frac{\mathcal{M}^{b}}{s^{2}} d t \asymp\left(\frac{c_{0}}{r}+c_{2} r+\cdots\right)\left(1+o\left(\varepsilon^{0}\right)\right) .
$$

Starting from the conditions (8.9, 8.10) we can now explain our method for satisfying the momentum cone conditions. Unfortunately, we cannot arrange that the contributions of the outer strip and the intermediate layers are of the form (8.9, 8.10), in particular because those contributions involve logarithms of $r$. However, using the same method as for the intermediate layers, we can construct an additional layer at $s \approx s_{4}$ with $0<s_{4} \ll$ $s_{3}$. This innermost layer should have the following properties. The contribution of the innermost layer to all the moments $I, J$, and $K^{(n)}$ should vanish as $\varepsilon \searrow 0$, so that the results of Propositions 6.1 and 7.1 remain valid. Moreover, its contribution to the integrals in $(8.9,8.10)$ should be of required form, and should be much larger than the corresponding contributions of the outer strip and the intermediate layers. Then the conditions (8.9, 8.10) are satisfied for the leading singular contributions, but the less singular contributions (in particular the contribution by the outer strip and the intermediate layers) will violate the momentum cone conditions. Then our strategy is to perturb the innermost layer by additional regularization tails so as to compensate for all the contributions to $\mathcal{M}\left[A_{x y}^{\varepsilon}\right]$ which violate the momentum cone conditions.

Before entering the details of the construction, we need to explain how in principle to compute the less singular contributions to the momentum cone conditions. First of all, there are the higher orders in the $s / r$ expansion, which were left out in (8.5) and (8.7); these terms are straightforward to compute. To explain the method for handling the tangential derivatives in (8.6), we consider as an example the contribution by the intermediate layer at $s_{2}$. Approximating it by a $\delta$-layer and for simplicity leaving out the log-terms, we obtain

$$
\mathcal{M}\left[A_{x y}^{\varepsilon}\right] \asymp\left(c_{1} \varepsilon^{-\frac{7}{128}} r^{-\frac{1+3 \alpha+\vartheta}{2 \alpha}} \frac{\not}{r}-b_{1} \varepsilon^{-\frac{1}{128}} r^{-\frac{3}{2}+\frac{1}{2 \alpha}} i \gamma^{0} \gamma^{r}\right) \delta\left(s-\varepsilon^{\frac{1}{16}} r^{\frac{1}{\alpha}}\right)
$$


and consequently

$$
r \mathcal{M}\left[A_{x y}^{\varepsilon}\right] \not z \asymp\left(2 c_{1} \varepsilon^{-\frac{7}{128}} r^{-\frac{1+\alpha+\vartheta}{2 \alpha}} s+i b_{1} \varepsilon^{-\frac{1}{128}} r^{-\frac{1}{2}+\frac{1}{2 \alpha}} \not\right) \delta\left(s-\varepsilon^{\frac{1}{16}} r^{\frac{1}{\alpha}}\right),
$$

where we omitted the higher orders in $s / r$. The tangential derivative $\partial_{t}+\partial_{r}$ is expressed in the coordinates $(s, r)$ simply by $\partial_{r}$. Hence by differentiating (8.11) with respect to $r$ and integrating with respect to $t$, we can compute the tangential derivative term in (8.6). The terms of higher order in $s / r$ as well as the correction terms arising from the finite size $\Delta s_{2}$ of the intermediate strip are straightforward to compute.

Finally, there are many correction terms which take into account all the simplifications made in the derivation of the momentum cone conditions. In order to treat these corrections systematically, we first note that to derive momentum cone conditions without any simplifications, instead of (4.2) we would have to consider the convolution integral

$$
B^{\varepsilon}:=\int \frac{d^{4} p}{(2 \pi)^{4}} \hat{\mathcal{M}}^{\varepsilon}(p) \hat{H}^{\varepsilon}(q-p),
$$

where $\hat{H}^{\varepsilon}$ is the high-energy part of the regularized fermionic projector,

$$
\hat{H}^{\varepsilon}(k)=\hat{P}^{\varepsilon}(k) \Theta\left(-k^{0}-\Omega\right) .
$$

The Fourier transform of $\hat{H}^{\varepsilon}$ can be determined in detail using the formulas of Lemma 5.1, and this makes it possible to compute $B^{\varepsilon}$ in position space in analogy to (4.8) by

$$
B^{\varepsilon}=\int e^{i \Omega t} \mathcal{M}\left[A_{x y}^{\varepsilon}\right] H^{\varepsilon}(\xi) d^{4} \xi .
$$

Let us be more specific, for simplicity only for the scalar component of $P^{\varepsilon}$ and for one Dirac sea. Then $H^{\varepsilon}$ is obtained from the formula of Lemma 5.1 simply by changing the integration range,

$$
H^{\varepsilon}(\xi)=\frac{i}{r} \int_{-\infty}^{-\Omega} d \omega h(\omega) e^{i \omega t}\left(e^{-i K(\omega) r}-e^{i K(\omega) r}\right) .
$$

Setting $h(\omega)=1 /\left(16 \pi^{3}\right)$ and $K=-\omega$, we recover our earlier formula for $H_{\text {scal }}$ (see (4.7)). Similar to Lemma 5.2, we can now perform the mass expansion,

$$
H^{\varepsilon}(\xi)=\frac{i}{r} \sum_{k=0}^{\infty} \int_{-\infty}^{-\Omega} d \omega h(\omega) e^{i \omega t}\left(e^{i \omega r} \frac{(i \alpha r)^{k}}{k !}-e^{-i \omega r} \frac{(-i \alpha r)^{k}}{k !}\right) .
$$

When computing the effect of the regularization terms, changing the integration range to the set $\Xi$ keeps the integral unchanged up to a contribution which tends to zero as $\varepsilon \searrow 0$. Therefore, the effect of the regularization terms is exactly as computed for $P^{\varepsilon}$ in Section 5 , up to small corrections which turn out to be negligible. Computing the unregularized contributions of higher order in the mass, we are led to integrals of the form

$$
\int_{-\infty}^{-\Omega} \frac{e^{i \omega(t \pm r)}}{\omega^{n}} d \omega
$$

By iteratively integrating by parts, one can reduce to the case $n=1$, which can be expressed in terms of the incomplete $\Gamma$ function

$$
\int_{-\infty}^{-\Omega} \frac{e^{i \omega x}}{\omega} d \omega=-\int_{\Omega x}^{\infty} \frac{e^{-i u}}{u} d u=-\Gamma(0, i \Omega x)
$$


and this can be asymptotically expanded in a straightforward way. Expanding $H^{\varepsilon}$ in this way, we obtain correction terms to $H_{\mathrm{scal}}^{\varepsilon}$ and similarly to $H_{\mathrm{vect}}^{\varepsilon}$. Computing the time integral of the resulting expressions, we get corrections to (4.15) and (4.17), which we refer to as the mass and regularization corrections to the momentum cone conditions.

We are now ready to state the main result of this section.

Proposition 8.1 We choose a parameter $\sigma$ in the range

$$
8<\sigma<9 .
$$

For any $c_{0}, c_{1} \in \mathbb{R}$, there is a family of fermionic projectors $\left(P^{\varepsilon}\right)_{\varepsilon>0}$ which satisfies the conditions of Propositions 6.1 and 7.1 and moreover has the following properties. For any $r$ in the range $r_{0}<r<r_{4}$ with

$$
r_{0} \gg \varepsilon^{\frac{3}{104}(3+2 \sigma)}, \quad r_{4} \ll \varepsilon^{-\frac{1}{832}(3+2 \sigma)},
$$

the layer

$$
\left|s-s_{4}\right|<\frac{\Delta s_{4}}{2} \quad \text { with } \quad s_{4}=r^{-\frac{13}{3+2 \sigma}} \varepsilon^{\frac{31}{64}}, \quad \Delta s_{4}=r^{-\frac{24+3 \sigma}{3+2 \sigma}} \varepsilon
$$

is an inner layer in the sense that it is vector dominated, whereas the regions outside this layer and in the range $\varepsilon \ll s<s_{3}$ are bilinear dominated. The family $\left(P^{\varepsilon}\right)_{\varepsilon>0}$ satisfies the momentum cone conditions, including the mass and regularization corrections.

Proof. We begin with the regularization functions as in the proof of Propositions 6.1 and 7.1 and keep adding regularization tails. We first make the ansatz

$$
\begin{aligned}
& \sum_{\beta=1}^{3} h_{\beta}(\omega) \asymp \kappa_{7} \hat{\mathrm{R}}^{p_{4}, q_{4}}\left(\varepsilon^{\frac{31}{64}}, \varepsilon^{\frac{7}{64}}, \sigma,-\omega\right) \\
& \sum_{\beta=1}^{3} g_{\beta}(\omega) \asymp \delta_{7} \hat{\mathrm{R}}^{p_{4}, q_{4}}\left(\varepsilon^{\frac{1}{2}}, \frac{6+17 \sigma}{13},-\omega\right) \\
& \sum_{\beta=1}^{3} f_{\beta}(\omega) \asymp \nu_{7} \hat{\mathrm{R}}^{p_{4}, q_{4}}\left(\varepsilon^{\frac{1}{2}}, \frac{3+15 \sigma}{13},-\omega\right)
\end{aligned}
$$

and choose $\nu_{7}$ such that the corresponding bilinear component (17.2) vanishes at $s_{4}$. Then we choose $\delta_{7}$ and $\kappa_{7}$ so as to satisfy the momentum cone conditions (8.9) and (8.10). More precisely, abbreviating the integrals in (8.9, 8.10) by

$$
X=\int_{0}^{\infty} \frac{\mathcal{M}^{0}}{s} d s, \quad Y=\int_{0}^{\infty} \frac{\mathcal{M}^{b}}{s^{2}} d s
$$

we arrange that the innermost layer gives the following contributions,

$$
X \sim r^{3} \varepsilon^{-\frac{59}{128}}, \quad Y \sim r \varepsilon^{-\frac{3}{16}} .
$$

The just determined parameters scale in $\varepsilon$ as follows,

$$
\kappa_{7} \sim \varepsilon^{\frac{62 \sigma-1}{128}}, \quad \delta_{7} \sim \varepsilon^{\frac{527 \sigma-48}{832}}, \quad \nu_{7} \sim \varepsilon^{\frac{465 \sigma-141}{832}} .
$$


Next we need to compensate for the singular contributions of the outer strip and the intermediate layers to $X$ and $Y$. We explain the method only for the most singular contributions; all the other terms can be compensated similarly. The leading contributions to $X$ and $Y$ comes from the intermediate strip at $s_{3}$,

$$
X \asymp r^{-\frac{2+3 \alpha+\vartheta}{2 \alpha}} \varepsilon^{-\frac{19}{128}}, \quad Y \asymp r^{\frac{2-3 \alpha+\vartheta+4 \tau+2 \vartheta \tau}{2 \alpha}} \varepsilon^{-\frac{21}{128}} .
$$

In order to compensate for these contributions, we proceed as follows. Introducing similar to (7.4) the notation

$$
a=A^{0}\left(s_{4}\right) \quad \text { and } \quad b=\left(A^{b}\right)^{\prime}\left(s_{4}\right)
$$

the contributions of the innermost layer to $X$ and $Y$ can be expressed by

$$
X(a, b) \asymp \frac{a^{2}}{b \sqrt{s_{4} r}}, \quad Y(a, b) \asymp \frac{a^{3}}{b^{2}\left(s_{4} r\right)^{\frac{3}{2}}} .
$$

By perturbing $a$ and $b$ one can easily perturb these contributions. It is convenient, however, to keep the zero $s_{4}$ of the bilinear component fixed, because perturbing $s_{4}$ would have a rather complicated influence on $a$ and $b$. We thus introduce the following tails,

$$
\begin{aligned}
\sum_{\beta=1}^{3} h_{\beta}(\omega) & \asymp \kappa_{8}\left[\hat{\mathrm{R}}^{p_{4}, q_{4}}\left(\varepsilon^{\frac{1}{2}}, \varepsilon^{\frac{7}{64}}, \vartheta_{h},-\omega\right)+l_{h 8} \hat{\mathrm{R}} \log ^{p_{4}, q_{4}}\left(\varepsilon^{\frac{1}{2}}, \varepsilon^{\frac{7}{64}}, \vartheta_{h},-\omega\right)\right] \\
\sum_{\beta=1}^{3} g_{\beta}(\omega) & \asymp \delta_{8}\left[\hat{\mathrm{R}}^{p_{4}, q_{4}}\left(\varepsilon^{\frac{1}{2}}, \vartheta_{g},-\omega\right)+l_{g 8} \hat{\mathrm{R}} \log ^{p_{4}, q_{4}}\left(\varepsilon^{\frac{1}{2}}, \vartheta_{g},-\omega\right)\right] \\
\sum_{\beta=1}^{3} f_{\beta}(\omega) & \asymp \nu_{8}\left[\hat{\mathrm{R}}^{p_{4}, q_{4}}\left(\varepsilon^{\frac{1}{2}}, \vartheta_{f},-\omega\right)+l_{f 8} \hat{\mathrm{R}} \log ^{p_{4}, q_{4}}\left(\varepsilon^{\frac{1}{2}}, \vartheta_{f},-\omega\right)\right] .
\end{aligned}
$$

We determine $\nu_{8}, \vartheta_{f}$ and $l_{f 8}$ such that the bilinear contribution of these tails vanishes at $s_{4}$. To compensate for the contribution to $X$ in (8.17), we then choose $\delta_{8}, \vartheta_{g}$ and $l_{g 8}$ such that the perturbation of $Y$ vanishes. We finally determine $\kappa_{8}, \vartheta_{h}$ and $l_{h 8}$ such that the perturbation of $X$ just cancels the term on the left of (8.17). Likewise, to compensate for $Y$ in (8.17), we can use the free parameters $\kappa_{8}, \vartheta_{h}$ and $l_{h 8}$.

Next we need to consider all the error terms. By choosing the parameters $p_{3}$ (in Proposition (7.1) and $q_{4}$ sufficiently large, we can arrange that the tails considered in Proposition 7.1 as well as the error terms of the tails considered in the proof of the present Proposition do not contribute to the moments in the layer at $s_{3}$. The error terms resulting from expansions in $\Delta s_{4} / s_{4}$ and in $s / r$ are all compensated in a straightforward way by suitable regularization tails.

It remains to satisfy the mass and regularization corrections to the momentum cone conditions. The regularization corrections tend to zero as $\varepsilon \searrow 0$. The mass corrections can be compensated in a straightforward manner. The only difficulty is that the tangential derivatives like in (8.6) destroy the general form (6.33) of the vector component. But the resulting radial error term can be compensated in a straightforward way by a contribution (7.9) which violates (6.33). 


\section{The Regularization Tails near the Origin}

In the calculations of Sections 6 8 we took into account only the leading order in $s / r$, and therefore these calculations do not apply near $r=0$. In this section we shall analyze the effect of the regularization tails near the origin. More precisely, we will compute $\mathcal{M}\left[A_{x y}^{\varepsilon}\right]$ asymptotically near $x=y$, and we will match these asymptotics to the outer strips and inner layers of the preceding sections. Our starting point is Lemma 5.1. Expanding in powers of $r$, all the negative powers of $r$ cancel, and we obtain to leading order

$$
\begin{aligned}
P^{\varepsilon}(x, y)= & \sum_{\beta=1}^{3} \int_{\Xi_{\beta}} 2 K_{\beta}(\omega) h_{\beta}(\omega) e^{i \omega t} d \omega+\mathcal{O}(r) \\
& +\gamma^{0} \sum_{\beta=1}^{3} \int_{\Xi_{\beta}} 2 K_{\beta}(\omega)\left(f_{\beta}(\omega)+\omega g_{\beta}(\omega)\right) e^{i \omega t} d \omega+\gamma^{0} \mathcal{O}(r) \\
& +\gamma^{r} \sum_{\beta=1}^{3} \int_{\Xi_{\beta}} \frac{2 i r}{3} K_{\beta}(\omega)^{3} g_{\beta}(\omega) e^{i \omega t} d \omega+\gamma^{r} \mathcal{O}\left(r^{2}\right) .
\end{aligned}
$$

Note that the leading radial contribution is linear in $r$. This fact, which can already be understood from the smoothness of $P^{\varepsilon}$ (note that $r \gamma^{r}=\vec{\xi} \vec{\gamma}$ is smooth, whereas $\gamma^{r}=\vec{\xi} \vec{\gamma} /|\vec{\xi}|$ is not), has an important consequence: Since at $r=0$ the radial component of $P^{\varepsilon}$ vanishes, the bilinear component of $A_{x y}^{\varepsilon}$ is also zero. The vector component of $A_{x y}^{\varepsilon}$, on the other hand, is proportional to $\gamma^{0}$. Combining these two observations, we conclude that the line $r=0$ is vector dominated.

Let us analyze in more detail how the outer strip and the inner layers behave asymptotically near the line $r=0$. The outer strip was constructed using the regularization tail (6.1), which led to a bilinear component of $A_{x y}^{\varepsilon}$, (6.8). The boundary $s_{1}$ of the outer strip separated the corresponding bilinear and vector dominated regimes (6.11) (all the other regularization tails in Section 6 affected the details of the outer strip, but are irrelevant here). Taking into account the regularization tail (6.1), the traceless part of the closed chain near the origin has the form

$$
\begin{aligned}
A_{x y}^{\varepsilon}-\frac{1}{4} \operatorname{Tr}\left(A_{x y}^{\varepsilon}\right) \asymp & -\frac{m^{3}}{64 \pi^{5}} \frac{\gamma^{0}}{|t|^{3}}(1+\mathcal{O}(r))+\gamma^{r} \mathcal{O}(r) \\
& +\frac{\delta r}{3 \pi^{3}} \epsilon(t) \frac{i \gamma^{0} \gamma^{r}}{|t|^{6+\gamma}} \gamma\left(1-\gamma^{2}\right) \cos (\pi \gamma / 2)(1+\mathcal{O}(r)) .
\end{aligned}
$$

The corresponding boundary between the vector and bilinear dominated regimes is given by

$$
r(t)=\frac{3 m^{3} \sec (\pi \gamma / 2)}{64 \pi^{2} \gamma\left(\gamma^{2}-1\right)} \frac{|t|^{3+\gamma}}{\delta}(1+\mathcal{O}(t)) .
$$

We thus obtain a vector dominated cone centered at the origin, which has zero opening angle. We refer to this cone as the vector dominated cusp at the origin. Our expansion near $r=0$ can be matched to the expansion in powers of $s / r$ as performed in Section 6 , Namely, the expansion (9.2) is valid if

$$
r \lesssim t \quad \text { and thus } \quad r, t \lesssim \delta^{\frac{1}{2+\gamma}} .
$$

The formula (6.11), on the other hand, holds in the range

$$
s \lesssim r \quad \text { and thus } \quad r \gtrsim \delta^{\frac{1}{2+\gamma}}
$$


We next discuss the effect of an additional scalar regularization tail of the form (6.18). Its contribution to the trace-free part of the closed chain is computed to be

$$
A_{x y}^{\varepsilon}-\frac{1}{4} \operatorname{Tr}\left(A_{x y}^{\varepsilon}\right) \asymp \frac{\kappa m}{\pi^{3}} \alpha \cos (\pi \alpha / 2) \gamma^{0}|t|^{-\alpha-4}+\mathcal{O}(r) .
$$

Assuming that $\alpha<\gamma+2$ and that $|t| \ll \kappa^{\frac{1}{1+\alpha}}$, from (9.1, 9.3) we again find a vector dominated cusp which scales like

$$
r(t) \sim \frac{\kappa}{\delta}|t|^{2+\gamma-\alpha} .
$$

Interestingly, we can arrange that the vector dominated cusp gives singular contributions to $\mathcal{M}\left[A_{x y}^{\varepsilon}\right]$ and/or to the momentum cone conditions, without affecting the outer strip and the inner layers. Here we merely explain the basic method, which will be very useful in order to compensate for singular contributions at the origin (see Section 11 for details). By choosing the vector and scalar tails (6.1, 6.18) appropriately, we want to arrange a contribution to $\mathcal{M}\left[A_{x y}^{\varepsilon}\right]$ near the origin $x \approx y$, without getting a contribution on the light cone away from the origin (in Section [11, (6.18) will be an additional scalar tail, and the following argument will make sure that this additional tail has no effect on the outer strip and the inner layers which are already present). Hence a neighborhood of the light cone should be bilinear dominated, and thus the bilinear component in (6.8) should dominate the vector component (6.21). This can be achieved most easily by choosing

$$
\alpha=\gamma+\frac{1}{2} \quad \text { and } \quad \kappa \ll \delta .
$$

Then the boundary of the vector dominated cusp has the scaling

$$
r(t) \sim \frac{\kappa}{\delta}|t|^{\frac{3}{2}}, \quad t(r) \sim\left(\frac{\delta r}{\kappa}\right)^{\frac{2}{3}},
$$

and thus

$$
\begin{aligned}
& r^{2} \int_{0}^{\infty} \mathcal{M}\left[A_{x y}^{\varepsilon}\right] d t \sim r^{2} \int_{t(r)}^{\infty} A^{0} d \tau \sim r^{2} \kappa t(r)^{-\alpha-3} \sim \kappa^{3+\frac{2 \alpha}{3}} \delta^{-2-\frac{2 \alpha}{3}} r^{-\frac{2 \alpha}{3}} \\
& r^{2} \int_{0}^{\infty} \frac{\mathcal{M}\left[A_{x y}^{\varepsilon}\right]}{t^{2}-r^{2}} d t \sim r^{2} \int_{t(r)}^{\infty} \frac{A^{0}}{\tau^{2}} d \tau \sim r^{2} \kappa t(r)^{-\alpha-5} \sim \kappa^{\frac{13+2 \alpha}{3}} \delta^{-\frac{10+2 \alpha}{3}} r^{-\frac{4+2 \alpha}{3}} .
\end{aligned}
$$

The point is that, after choosing $\alpha$ sufficiently large, we obtain non-integrable poles at $r=$ 0 . By arranging that the scalar tail is present up to some small radius $\tilde{r}>0$, we can thus make the contribution of the vector dominated cusp to the above expressions arbitrarily large in the distributional sense.

We next analyze the inner layers near the origin. We thus consider the tails (7.1). Near the origin, the bilinear component of the closed chain becomes

$$
A^{b}=-\frac{\delta}{3 \pi^{3}} \gamma\left(\gamma^{2}-1\right) \cos (\pi \gamma / 2) r|t|^{-6-\gamma} \epsilon(t)+\frac{\nu}{\pi^{3}} \beta \sin (\pi \beta / 2) r|t|^{-5-\beta} \epsilon(t)+\mathcal{O}\left(r^{2}\right) .
$$

The zero of the bilinear component is at fixed time

$$
t=\left(\frac{3 \beta \sin (\pi \beta / 2)}{\gamma\left(\gamma^{2}-1\right) \cos (\pi \gamma / 2)} \frac{\nu}{\delta}\right)^{\frac{1}{\beta-\gamma-1}} .
$$

This asymptotic form is again consistent with the expansion in powers of $s / r$, (7.3). Taking into account the scalar tail (6.18), we obtain again a vector dominated region near $r=0$. The relevant scalings are shown in Figure 7 , where we also indicate the possible singular contributions at the origin by a white circle. 


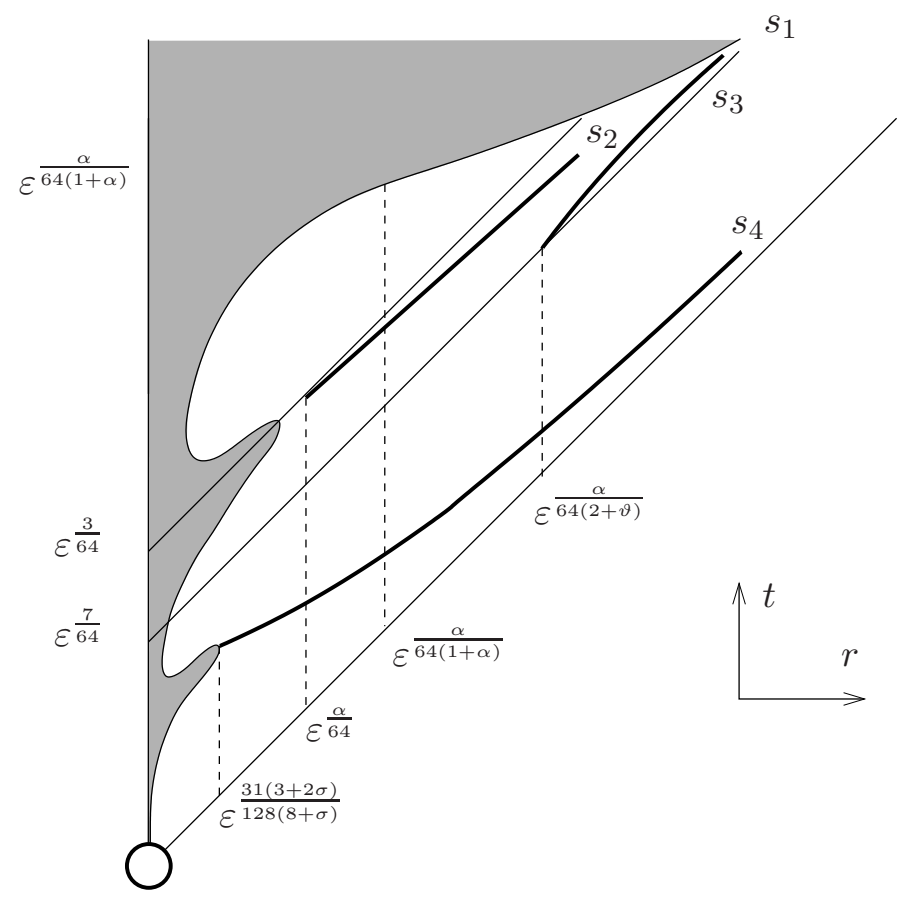

Figure 7: Behavior of $\mathcal{M}\left[A_{x y}^{\varepsilon}\right]$ near the origin.

\section{The Regularization Tails near Infinity}

The structure of the outer strip and of the inner layers persists only up to maximal radii $r_{1}, \ldots, r_{4}$ (see Propositions [6.1, 7.1] and 8.1), and therefore we must analyze the behavior of $\mathcal{M}\left[A_{x y}^{\varepsilon}\right]$ for even larger radii. The basic difficulty for large $\xi$ can already be understood by considering the outer layer of Proposition 6.1, whose boundary is given by

$$
s_{1}=\varepsilon^{\frac{1}{64}} r^{-\frac{1}{\alpha}} .
$$

In Section 6 we made use of the mass expansion, and thus (10.1) holds only if $s_{1} r \ll m^{-2}$. This leads to the condition

$$
\varepsilon^{\frac{1}{64}} r^{1-\frac{1}{\alpha}} \ll m^{-2} \quad \text { or equivalently } \quad r \ll \varepsilon^{-\frac{\alpha}{64(\alpha+1)}} .
$$

Thus if $r \gg r_{1}$, the bilinear dominated regime enters the region $r s \gg m^{-2}$ where the fermionic projector has an oscillatory behavior. These oscillations of $P(x, y)$ also lead to an oscillatory behavior of the closed chain, making it difficult to describe the bilinear dominated region explicitly.

In order to bypass this difficulty, we shall now modify the fermionic projector by changing the direction of the vector field $\not k$ in (5.1). In agreement with the more general notion introduced in [6, $\S 4.4]$, we refer to this mechanism as introducing a shear of the surface states. The basic idea already becomes clear if we consider the unregularized fermionic projector and "shorten" the Dirac matrix $\gamma^{0}$ by the transformation

$$
\gamma^{0} \longrightarrow(1-\theta) \gamma^{0}
$$


with a positive parameter $\theta \ll 1$. Clearly, this transformation does not change the singular set $t= \pm r$ of the distribution $P(x, y)$. But it does change the square of the matrix $\not$,

$$
(\not{\phi})^{2} \longrightarrow(1-\theta)^{2} t^{2}-r^{2} .
$$

As a consequence, computing $\mathcal{M}\left[A_{x y}\right]$ naively similar to (2.20), we find that

$$
\mathcal{M}\left[A_{x y}\right]=0 \quad \text { if } \quad|t|<\frac{r}{1-\theta} .
$$

In particular, $\mathcal{M}\left[A_{x y}\right]$ now vanishes identically in a neighborhood of the light cone. For our purpose, it is very helpful that the boundary of the region where $\mathcal{M}\left[A_{x y}\right]$ vanishes is easy to describe: it consists simply of the two cones $t= \pm r /(1-\theta)$.

The transformation (10.2) can also be expressed by choosing the functions $f_{\beta}$ in the spherically symmetric regularization (5.1) as

$$
f_{\beta}(\omega)=-\frac{\delta}{16 \pi^{3}} \omega
$$

This transformation is too simple for our application, because we want (10.2) to be active only in the region $s \gg \epsilon^{\frac{3}{8}}$, so that it affects the outer strip, but not the inner layers. To this end, we choose

$$
r_{\infty}=\varepsilon^{-\frac{\alpha}{64}}, \quad \theta=\frac{s_{1}\left(r_{\infty}\right)}{r_{\infty}}=\varepsilon^{\frac{1}{32}+\frac{\alpha}{64}}
$$

and introduce a contribution to the functions $f_{\beta}$ of the form

$$
f_{\beta} \asymp-\frac{\theta}{16 \pi^{3}} \hat{\mathrm{R}}^{p_{\infty}, q_{\infty}}\left(\varepsilon^{\frac{3}{64}}, 2, \omega\right),
$$

where $p_{\infty}, q_{\infty}$ are integer parameters. This has the following effect. If $s \ll \varepsilon^{\frac{3}{64}}$, the Fourier transform $\hat{\mathrm{R}}^{p_{\infty}, q_{\infty}}(s)$ of $\mathrm{R}^{p_{\infty}, q_{\infty}}(s)$ decays like $\left(s / \varepsilon^{\frac{3}{64}}\right)^{p_{\infty}}$ (see Lemma 5.6] and Figure 4). Thus by by choosing $p_{\infty}$ sufficiently large we can arrange that (10.4) has no effect on the inner layers. However, if $s \ll \varepsilon^{\frac{3}{64}}$, only the behavior of $\mathrm{R}^{p_{\infty}, q_{\infty}}$ for $\omega \ll \varepsilon^{-\frac{3}{64}}$ is relevant. Considering the asymptotics of (5.13) for small $|\omega|$, we get agreement with (10.3), with an error term which can be made arbitrarily small by increasing $q_{\infty}$. Hence the regularization functions (10.4) influence the outer strip exactly as (10.3), but have no effect on the inner layers.

In order to quantify the influence of (10.4) on the outer strip, we now compute the boundary $s_{1}$ of the bilinear and vector dominated regions in the asymptotic regime

$$
\varepsilon^{-\frac{\alpha}{64}}=r_{\infty} \ll r \ll\left(m^{2} \theta\right)^{-\frac{1}{2}}=\frac{1}{m} \varepsilon^{-\frac{1}{64}-\frac{\alpha}{128}},
$$

where $\theta$ plays in important role, but nevertheless the mass expansion can be used. In this regime (6.8) is modified to

$$
\begin{aligned}
A_{x y}^{\varepsilon}-\frac{1}{4} \operatorname{Tr}\left(A_{x y}^{\varepsilon}\right) \asymp & \frac{\delta}{8 \pi^{3}}(1-\theta) \frac{i \gamma^{0} \gamma^{r}}{r^{3}}(1-\gamma)|s|^{-\gamma-2} \cos (\pi \gamma / 2) \\
& -\frac{m^{3}}{256 \pi^{5}} \frac{1}{r^{2} s^{2}} \Theta(s)\left((1-\theta) t \gamma^{0}-r \gamma^{r}\right) .
\end{aligned}
$$




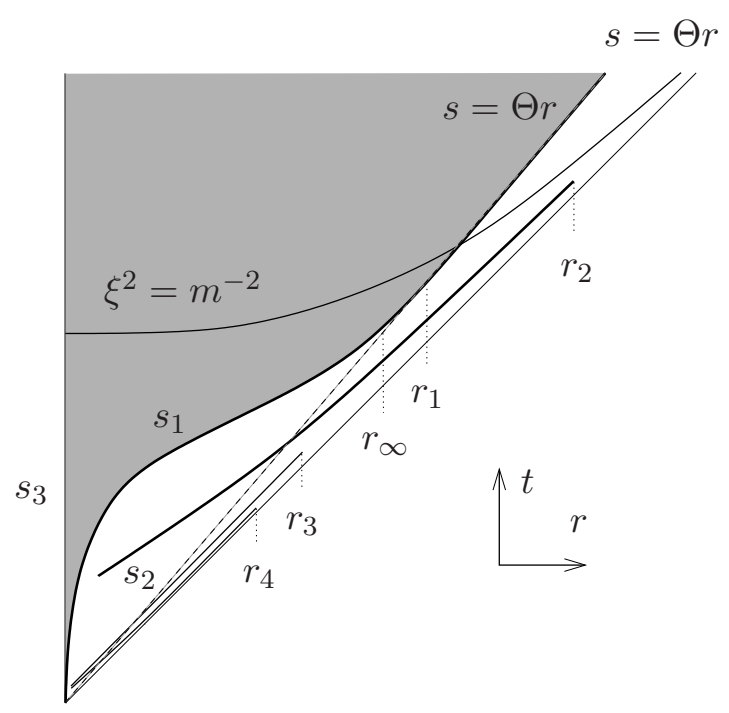

Figure 8: Behavior of $\mathcal{M}\left[A_{x y}^{\varepsilon}\right]$ near infinity.

Writing $s=\theta r+\Delta s$, the correction $\Delta s$ is to leading order determined from the equation

$$
\delta \frac{(\theta r)^{-\gamma-2}}{r^{3}} \sim \frac{1}{r^{2}(\theta r)^{2}} \sqrt{\Delta s r}
$$

and thus

$$
s_{1}=\theta r+\delta^{2} r^{-2 \gamma-3} \theta^{-2 \gamma}+\cdots .
$$

This shows that the function $s_{1}(r)-\theta r$ tends to zero fast and becomes negligible before $s_{1}$ enters the oscillatory region of the fermionic projector, see Figure 8 ,

We next consider the behavior of the inner layers for large $r$. According to the above construction, they are not affected by the regularization tail (10.4). The layers at $s_{2}$ and $s_{4}$ have the property that $s$ decreases for increasing $r$ (see (7.13) and (8.12)). According to (7.15, 8.13), we choose the length scale of the scalar regularization tail such that this tail dies off at a smaller radius than the vector tails. As a consequence, the corresponding inner layers "fade away smoothly" on the scales $r_{2}$ and $r_{4}$, respectively. For the layer at $s_{3}$, the situation is somewhat different because $s_{3}$ increases with $r$. Due to the second scale in (7.19), the corresponding scalar regularization tail dies off if $s \gtrsim \varepsilon^{\frac{11}{128}}$ (see the right of Figure (4). We thus conclude that the inner layer at $s_{3}$ also fades away smoothly on the scale $r_{3}$.

We finally remark that, by changing the scales of the scalar regularization tails 7.15 , 7.19, 8.13), we could still modify the radii $r_{2}, r_{3}$ and $r_{4}$. But we will not need this freedom here.

\section{The Continuum Limits of $\mathcal{M}$ and $\mathcal{M} \cdot P$}

We are now ready to prove the main theorem.

Proof of Theorem 2.3. We consider a family of regularizations $\left(P_{\varepsilon}\right)_{\varepsilon>0}$, to which Propositions 6.1 7.1 and 8.1 apply, and in addition the shear of the surface states (10.4) is 
present. Then clearly (1) holds. To prove (2), for a given test function $f \in \mathcal{S}\left(\mathbb{R}^{4}\right)$ we must show that

$$
\lim _{\varepsilon \searrow 0} \int\left(\mathcal{M}\left[A_{x y}^{\varepsilon}\right]-\tilde{\mathcal{M}}(\xi)\right) f(\xi) d^{4} \xi=0
$$

In order to first analyze the situation in a compact set away from the origin, we consider for given parameters $\delta, \gamma \geq 0$ the integral

$$
\int\left(\mathcal{M}\left[A_{x y}^{\varepsilon}\right]-\tilde{\mathcal{M}}(\xi)\right) f(\xi)\left(\eta_{\varepsilon^{-\gamma}}(\xi)-\eta_{\delta}(\xi)\right) d^{4} \xi
$$

Obviously, in any compact set away from the light cone, the function $\mathcal{M}\left[A_{x y}^{\varepsilon}\right]$ tends to $\tilde{\mathcal{M}}(\xi)$ as $\varepsilon \searrow 0$. Moreover, comparing Lemma 3.1 with Propositions 6.1 and 7.1 , one sees that $\mathcal{M}\left[A_{x y}^{\varepsilon}\right]-\tilde{\mathcal{M}}(\xi)$ tends to zero on the light cone in the distributional sense. This means that for every $\delta>0$ and after choosing $\gamma=0$,

$$
\lim _{\varepsilon \searrow 0} \int\left(\mathcal{M}\left[A_{x y}^{\varepsilon}\right]-\tilde{\mathcal{M}}(\xi)\right) f(\xi)\left(\eta_{\varepsilon^{-\gamma}}(\xi)-\eta_{\delta}(\xi)\right) d^{4} \xi=0 .
$$

Since all the scales in our regularization are powers of $\varepsilon$, (11.2) remains valid if $\gamma$ is chosen positive, but sufficiently small.

We next consider the integral

$$
\int\left(\mathcal{M}\left[A_{x y}^{\varepsilon}\right]-\tilde{\mathcal{M}}(\xi)\right) f(\xi)\left(1-\eta_{\varepsilon^{-\gamma}}(\xi)\right) d^{4} \xi
$$

The contribution by $\tilde{M}$ clearly tends to zero as $\varepsilon \searrow 0$. To control the contribution by $\mathcal{M}\left[A_{x y}^{\varepsilon}\right]$, we first note that $\mathcal{M}\left[A_{x y}^{\varepsilon}\right]$ is obviously bounded by a negative power of $\varepsilon$, i.e. there is $n \in \mathbb{N}$ such that

$$
\left\|\mathcal{M}\left[A_{x y}^{\varepsilon}\right]\right\| \leq C \varepsilon^{-n} \quad \text { for all } \xi .
$$

This negative power of $\varepsilon$ is compensated in (11.3) by the rapid decay of $f$. We conclude that

$$
\lim _{\varepsilon \searrow 0} \int\left(\mathcal{M}\left[A_{x y}^{\varepsilon}\right]-\tilde{\mathcal{M}}(\xi)\right) f(\xi)\left(1-\eta_{\varepsilon^{-\gamma}}(\xi)\right) d^{4} \xi=0
$$

To finish the proof of (2) it remains to show that

$$
\lim _{\delta \searrow 0} \lim _{\varepsilon \searrow 0} \int\left(\mathcal{M}\left[A_{x y}^{\varepsilon}\right]-\tilde{\mathcal{M}}(\xi)\right) f(\xi) \eta_{\delta}(\xi) d^{4} \xi=0
$$

because (11.1) then follows immediately by combining (11.2, 11.4, 11.5). In view of Lemma 3.1, we only need to consider the vector dominated cusps, see (9.2) and Figure 7 The leading contributions to the integral in (11.5) have the following scaling,

$$
\int\left(\mathcal{M}\left[A_{x y}^{\varepsilon}\right]-\tilde{\mathcal{M}}(\xi)\right) f(\xi) \eta_{\delta}(\xi) d^{4} \xi \sim A^{t} t r^{3} f(0)+A^{b} t r^{4} \nabla f(0) .
$$

A short calculation shows that all these contributions tend to zero as $\varepsilon \searrow 0$. This concludes the proof of (2).

Next we want to show that, for any $k$ not on the mass cone, $\hat{\mathcal{M}}^{\varepsilon}(k)$ converges even pointwise,

$$
\lim _{\varepsilon \searrow 0} \hat{\mathcal{M}}^{\varepsilon}(k)=\tilde{\mathcal{M}}(k) \quad \text { if } k^{2} \neq 0 .
$$


To this end, we consider the Fourier integral

$$
\hat{\mathcal{M}}^{\varepsilon}(k)=\int \mathcal{M}\left[A_{x y}^{\varepsilon}\right] e^{-i k \xi} d^{4} \xi
$$

Choosing polar coordinates $(t, r, \vartheta, \varphi)$ and carrying out the angular variables as in the proof of Lemma 5.1, we obtain an expression involving two-dimensional Fourier transforms of the following form:

$$
\int_{-\infty}^{\infty} d t \int_{0}^{\infty} r^{p} d r \mathcal{M}^{\varepsilon}(t, r) e^{-i \omega t \pm i k r}, \quad p \in \mathbb{N}_{0}
$$

For $t$ and $r$ in a compact set, we can argue exactly as in (11.2) and (11.5). Hence it remains to consider the integral near infinity,

$$
\int_{0}^{\infty} r^{p} d r e^{-i(\omega \mp k) r} \eta_{\varepsilon^{-\gamma}}(r) \int_{-\infty}^{\infty} d s e^{-i \omega s} \mathcal{M}^{\varepsilon}(t, r)
$$

where we again chose the light-cone coordinate $s=t-r$. Since $\omega \mp k \neq 0$, we can use the identity

$$
e^{-i(\omega \mp k) r}=\frac{i}{\omega \mp k} \frac{d}{d r} e^{-i(\omega \mp k) r}
$$

and integrate by parts in the variable $r$. This gives a scaling factor $\varepsilon^{\gamma}$. We iterate this procedure until the expression scales like a positive power of $\varepsilon$. Then we can take the limit $\varepsilon \searrow 0$ to obtain (11.7).

For the proof of (3) we need to analyze similar to (2.25) the following convolution integral,

$$
\int \frac{d^{4} p}{(2 \pi)^{4}} \hat{\mathcal{M}}^{\varepsilon}(p) P^{\varepsilon}(q-p) .
$$

If $P^{\varepsilon}$ were replaced by a family of distributions with compact support, the convolution integral as well as the limit $\varepsilon \searrow 0$ would be well defined as the convolution of distributions. Using furthermore the pointwise convergence (11.7), one sees that it is indeed sufficient to verify the momentum cone conditions. In Proposition 8.1, these conditions were satisfied on the light cone, in an annulus $\delta<r<\varepsilon^{-\gamma}$. For large $r>\varepsilon^{-\gamma}$ we can in (4.14, 4.16) iteratively substitute the identity

$$
e^{-i \Omega r}=\frac{i}{\Omega} \frac{d}{d r} e^{-i \Omega r}
$$

and integrate by parts to show that the corresponding contribution to the convolution integral (11.8) tends to zero as $\varepsilon \searrow 0$. Hence it remains to consider the momentum cone conditions near the origin $\xi=0$. Using the symmetries (8.2, 8.3), the leading contributions have the following form,

$$
\begin{aligned}
B_{\mathrm{scal}}^{\varepsilon} & \sim \frac{A^{t}}{t^{2}} t r^{3}+\frac{A^{b} r^{2}|\vec{k}|}{t^{2}} t r^{3} \\
B_{\mathrm{vect}}^{\varepsilon} & \sim \frac{A^{b} r}{t^{4}} t r^{3}+\frac{A^{b} r \Omega|\vec{k}|}{t^{2}} t r^{3}
\end{aligned}
$$


These contributions actually diverge as $\varepsilon \searrow 0$, but we can compensate for them using the mechanism explained after (9.3). More precisely, we introduce the additional tails

$$
\begin{aligned}
& \sum_{\beta=1}^{3} h_{\beta}(\omega) \asymp \kappa_{1} \hat{\mathrm{R}}^{p, q}\left(\varepsilon, \varepsilon^{\frac{45}{192}}, \frac{13}{8},-\omega\right)+\kappa_{2} \hat{\mathrm{R}}^{p, q}\left(\varepsilon^{\frac{45}{192}}, \varepsilon^{\frac{43}{192}}, \frac{13}{8},-\omega\right) \\
& \sum_{\beta=1}^{3} g_{\beta}(\omega) \asymp \delta_{1} \hat{\mathrm{R}}^{p, q}\left(\varepsilon, \varepsilon^{\frac{45}{192}}, \frac{9}{8},-\omega\right)+\delta_{2} \hat{\mathrm{R}}^{p, q}\left(\varepsilon^{\frac{45}{192}}, \varepsilon^{\frac{43}{192}}, \frac{9}{8},-\omega\right),
\end{aligned}
$$

and also introduce log-tails, with the relative prefactor chosen exactly as in the tails leading to the singularities (11.9, 11.10). Since the log-tails are straightforward, we do not consider them here. By choosing the parameters $\kappa_{i}$ and $\delta_{i}$ appropriately, these tails give rise to additional vector dominated cusps at $t \sim \varepsilon^{\frac{11}{48}}$ and $t \sim \varepsilon^{\frac{23}{96}}$, which do not contribute to (11.6), but compensate for the leading terms in (11.9, 11.10). After choosing $p$ and $q$ sufficiently large, all the correction terms can be treated by perturbing these two vector dominated cusps. The parameters $\kappa_{i}$ and $\delta_{i}$ can be chosen to have the following scaling in $\varepsilon$,

$$
\kappa_{1} \sim \varepsilon^{\frac{3977}{2688}}, \quad \delta_{1} \sim \varepsilon^{\frac{1625}{896}}, \quad \kappa_{2} \sim \varepsilon^{\frac{1255}{768}}, \quad \delta_{2} \sim \varepsilon^{2} .
$$

Finally, we need to take into account that the contributions of the innermost layer to the momentum cone conditions (․16) cease to exist if $r \lesssim \varepsilon^{\frac{5(3+2 \sigma)}{832}}$ (see Figure 7). However, this "missing contribution" to $B^{\varepsilon}$ tends to zero as $\varepsilon \searrow 0$. This concludes the proof of (3).

To prove (4), we note that for $q \notin \overline{\mathcal{C}^{\wedge}}$, the integration range in the convolution integral (2.25) becomes unbounded (cf. Figure 1). For large $p$, the integrand in (2.25) is easily computed to be a positive definite matrix. The same is true for the regularized integrand in (11.8), showing that the convolution integral (11.8) diverges as $\varepsilon \searrow 0$ to $+\infty$.

\section{Going Beyond the Distributional $\mathcal{M} \cdot P$-Product}

We saw in Section 9 that the regularization tails give us a lot of freedom to modify $\mathcal{M}\left[A_{x y}^{\varepsilon}\right]$ near the origin. We now use this freedom to go beyond the distributional $\mathcal{M} P$-product.

Proof of Theorem 2.4. Our task is to arrange additional contributions near the origin which leave the continuum limit of $\mathcal{M}\left[A_{x y}^{\varepsilon}\right]$ unchanged but in the product (2.8) give rise to the extra contribution

$$
\frac{1}{2} \lim _{\varepsilon \searrow 0} \mathcal{M}\left[A_{x y}^{\varepsilon}\right] P^{\varepsilon}(x, y) \asymp c_{2} \delta^{4}(\xi)-c_{3} i \not \partial \delta^{4}(\xi)-c_{4} \square \delta^{4}(\xi) .
$$

Our method is similar to that discussed in Section 9 after (9.3). The main difference is that instead of working with the tails $g$ and $h$, it is preferable here to use the tails $f$ and $h$. More precisely, we consider a scalar regularization tail of the form (6.18) as well as the vector tail

$$
f(\omega) \asymp e^{\varepsilon \omega} \frac{\nu}{\Gamma\left(\alpha-\frac{1}{2}\right)}|\omega|^{\alpha-\frac{1}{2}-1} \Theta(-\omega) .
$$

Choosing

$$
\kappa \ll \nu,
$$


the region near the light cone is bilinear dominated, and thus adding the above tails to the tails already considered earlier, in such a way that the new tails are active for even smaller values of $s$, the multilayer structure near the light cone is not affected. Near the origin, we get a vector dominated cusp of the form

$$
r \sim \frac{\kappa}{\nu} \sqrt{|t|} .
$$

We arrange the tails to be active on the scale $|t| \sim \tau$. Then for the regularization tails to be small, we must satisfy the conditions

$$
\kappa \ll \tau^{-1+\alpha}, \quad \nu \ll \tau^{-\frac{3}{2}+\alpha} .
$$

Choosing $\kappa$ and $\tau$ according to this optimal scaling, we clearly satisfy (12.3), whereas (12.4) yields $r \sim \tau$. This shows that we can arrange that the vector dominated cusp has a nonzero opening angle on the scale $\tau$. On this scale, we have the following asymptotic forms for composite expressions

$$
\begin{aligned}
A_{x y}^{\varepsilon}-\frac{1}{4} \operatorname{Tr}\left(A_{x y}^{\varepsilon}\right) & \asymp \not \& r_{0} \kappa \tau^{-5-\alpha} \epsilon(t)+\gamma^{0} r_{1} \nu \tau^{-\frac{5}{2}-\alpha} \\
P^{\varepsilon}(x, y) & \asymp i \not \& r_{2} \tau^{-4}+\gamma^{0} c_{0} \nu \tau^{-\frac{1}{2}-\alpha}+r_{3} \tau^{-2}+\kappa c_{1} \tau^{-1-\alpha}
\end{aligned}
$$

with real coefficients $r_{j}$ and complex coefficients $c_{j}$. By direct inspection one sees that we can arrange terms which have the same symmetry as the terms in (12.1) and the correct scaling; these terms are of the form $\sim \not \kappa^{2}$ and $\sim \nu \kappa$. Moreover, we have the freedom to choose an arbitrarily large number of pairs of tails in $f$ and $h$ active on different scales $\tau$. Using all this freedom, it is straightforward to verify that there are regularizations having all the required properties.

We remark that there is an alternative method for proving Theorem 2.4. Namely, instead of considering the regularization tails, one can work with additional high-energy states, which are not close to the mass cone or are no surface states (for definitions and a discussion see [6, Chapter 4]). Actually, working with the high-energy states gives us more flexibility in modifying $\mathcal{M}\left[A_{x y}^{\varepsilon}\right]$ near the origin. However, at least for regularizations which have the natural scaling (see for example [6, eqn (4.3.12)]), we get the same results as with the regularization tails. For this reason, here we shall not enter the construction of the high-energy states.

It is an obvious question whether one can arrange additional contributions to $Q$ supported on the light cone. More specifically, from the scaling the following contributions seem possible,

$$
\begin{array}{lll}
\sim \not \delta \delta\left(\xi^{2}\right) \epsilon\left(\xi^{0}\right) & , & \sim \delta\left(\xi^{2}\right) \epsilon\left(\xi^{0}\right) \\
\sim i \not \& \delta\left(\xi^{2}\right) & , & \sim \delta\left(\xi^{2}\right) .
\end{array}
$$

Computing the Fourier transform, the contributions (12.6) are supported on the mass cone $\left\{k^{2}=0\right\}$ and are thus not of interest. The terms (12.7), however, do contribute in the lower masse cone; more precisely, the corresponding contribution to $\hat{Q}$ can be written as

$$
\hat{Q}(k) \asymp c_{5} \frac{\not k}{k^{4}}+c_{6} \frac{1}{k^{2}} \quad\left(k \in \mathcal{C}^{\wedge}\right)
$$

with real constants $c_{5}$ and $c_{6}$. A potential method for arranging these extra contributions would be to construct additional layers whose contribution to $\mathcal{M}\left[A_{x y}^{\varepsilon}\right]$ vanishes as $\varepsilon \searrow 0$, 
but which, when multiplied by $P^{\varepsilon}(x, y)$, give rise to the terms (12.7). Although we have no proof, we conjecture that this cannot be accomplished. In any case, arranging the extra terms on the light cone (12.7) is much more difficult than arranging the terms at the origin (12.1). One difficulty is that for contributions on the light cone one must get the scaling in the radius right. Furthermore, a symmetry argument shows that the product $\mathcal{M}\left[A_{x y}^{\varepsilon}\right] P(x, y)$ can only give rise to the uninteresting contributions (12.6). Thus to obtain (12.7) one needs to take into account the regularization tails of the factor $P^{\varepsilon}(x, y)$ in the product $\mathcal{M}\left[A_{x y}^{\varepsilon}\right] P^{\varepsilon}(x, y)$. This goes beyond our considerations used for the momentum cone conditions and does not seem easy.

Finally, it is a good question why at all we want to implement the distributional $\mathcal{M} P$-product. This property is very useful because it makes it possible to analyze the Euler-Lagrange equations in the continuum, but this is certainly not a compelling reason for imposing it. Another fair approach would be to consider right away the regularized product

$$
Q^{\varepsilon}(x, y)=\frac{1}{2} \mathcal{M}\left[A_{x y}^{\varepsilon}\right] P^{\varepsilon}(x, y),
$$

and to try to choose the regularization tails such as to give the singularities of (12.8) on the light cone a meaning in the distributional sense. We strongly conjecture that this procedure does not work, although we again have no proof. Namely, giving (12.8) a distributional meaning leads to the following serious difficulties. First, the pole of (12.8) on the light cone is of higher order than that of $\mathcal{M}$, and thus we would have to introduce more counterterms. Second, for fixed $x$ and $y,(12.8)$ is a non-selfadjoint matrix which also involves a scalar component. This again increases the number of counterterms by more than a factor of two. In view of the difficulties encountered in Sections 66 8, it seems hopeless to construct all these counterterms. This argument gives a justification for the assumption of a distributional $\mathcal{M} P$-product which does not refer to aesthetics nor usefulness: this assumption drastically simplifies the structure of the singularities on the light cone which need to be given a distributional meaning.

\section{General Remarks}

In the preceding constructions we worked with a special class of regularizations. This raises the following questions:

(1) Considering only our restrictive class of regularizations satisfying Theorem 2.3, is there still enough freedom to fulfill additional constraints? More specifically, is it possible to satisfy the condition of half-occupied surface states? Are the regularization parameters still linearly independent? Can the fermionic states still be properly normalized?

(2) To what extent was our choice of regularizations only a matter of convenience, and to what extent a matter of necessity? Clearly, many details of the regularization could be modified, but are there general properties which are canonical? In more physical terms (assuming that a suitably regularized fermionic projector describes nature), what do we learn about the microscopic structure of space-time?

In this section we answer or at least discuss these questions. 
Remark 13.1 (half occupied surface states and the normalization of the fermionic states) In the constructions of Sections 6 11, we imposed conditions on the following combinations of regularization functions,

$$
\sum_{\beta=1}^{3} h_{\beta}, \quad \sum_{\beta=1}^{3} \alpha_{\beta} h_{\beta}, \quad \sum_{\beta=1}^{3} g_{\beta}, \quad \sum_{\beta=1}^{3} \alpha_{\beta} g_{\beta}, \quad \sum_{\beta=1}^{3} f_{\beta}
$$

(see (6.41 6.50), (7.15 7.17), (7.19 (7.21), (77.22), (7.23), (8.13 8.15), (8.18 8.20), (10.4), (11.11(11.12)). Moreover, we implicitly assumed that

$$
\sum_{\beta=1}^{3} \alpha_{\beta} f_{\beta}=0
$$

The higher orders in the mass expansion are so small near the light cone that they do not contribute in the limit $\varepsilon \searrow 0$. Hence in the construction of the tails we prescribed six functions of $\omega$. On the other hand, the regularization of each of the three Dirac seas involves the four free functions $f_{\beta}, g_{\beta}, h_{\beta}$ and $\alpha_{\beta}$. Thus for every $\omega$, we have twelve free parameters to satisfy six conditions. This leaves us with six free parameters, two for each Dirac sea. This is precisely what we need to satisfy the condition of half occupied surface states (5.4) and to normalize the fermionic states according to [6, $\S 2.6]$ :

- In a system with three generations, there is a family of regularizations $\left(P_{\varepsilon}\right)_{\varepsilon>0}$ having all the properties in Theorem 2.3, and furthermore the surface states are half occupied with properly normalized fermions.

It is remarkable that the situation would be completely different if we had worked with less than three generations. More precisely, counting the number of free parameters, one immediately gets the following results:

- In a system with two generations, there is a family of regularizations $\left(P_{\varepsilon}\right)_{\varepsilon>0}$ having all the properties in Theorem 2.3, and furthermore the surface states are half occupied or properly normalized.

- In a system with one generation, it does not seem possible to regularize the fermionic projector in such a way that Theorem 2.3 holds.

This consideration gives a natural explanation why three generations of elementary particles appear in nature.

Remark 13.2 (linear independence of the regularization parameters)

In the continuum limit [6, Chapter 4], the unknown microscopic structure of space-time is taken into account by a small number of free parameters, the so-called regularization parameters. In [6, Appendix E] it was shown by an explicit construction of suitable regularizations that the regularization parameters are linearly independent. It is an important question whether this result remains true for the more restrictive class of regularizations considered here. To answer this question, we first note that in [6. Appendix E] we worked with the moments of the regularization functions, such as the quantities

$$
\int_{0}^{\infty} \omega^{n} h_{\alpha}(\omega) d \omega
$$


whereas in the preceding sections we analyzed the tails of the regularization. The tails and the moments describe complementary properties of the regularization functions, which can can be prescribed independently. Therefore, the considerations of the tails of the present paper are not in conflict with the considerations in [6, Appendix E]. Writing down families of regularization functions which satisfy both Theorem 2.3 and [6, Theorem E.1] seems straightforward (although the explicit formulas would clearly become rather complicated).

Alternatively, this result can be understood in position space as follows. The regularization tails are active in the region $s \gg E_{P}$. The regularization parameters, on the other hand, model the behavior of the fermionic projector with interaction near the light cone $s \sim E_{P}$. Since the range of $s$ is different in both cases, it is clear that the tails do not affect the regularization parameters.

To avoid misunderstandings, we repeat the warning from [6, Appendix E] that linear independence of the regularization parameters does not mean that, by choosing suitable regularization functions, the regularization parameters can be given arbitrary values. The regularization parameters might well be constrained by certain inequalities. For this reason, in applications one should always verify that the values for the regularization parameters needed in the effective continuum theory can actually be realized by suitable regularization functions.

Remark 13.3 (universal properties of the regularizations)

Clearly, many construction steps in this paper were arbitrary or merely a matter of convenience. Nevertheless, based on the experience of numerous calculations involving different kinds of regularization functions, it seems that a few properties of our regularizations are universal in the sense that every admissible family of regularizations should have these properties. More precisely, we can make the following general considerations.

- The structure of our variational principle leads us to distinguish between vector and bilinear dominated regions. In order to get correspondence to Minkowski space, the boundary between these regions must be close to the light cone, and it might involve a transition region with one or several layers. The property of a distributional $\mathcal{M P}$-product gives so many conditions that it seems impossible to satisfy all these conditions with only one layer. This means that every admissible regularization should have a multilayer structure near the light cone.

- At first sight, it might seem a promising strategy to make the regularization "as Lorentz invariant as possible" by arranging that the boundary between the vector and bilinear dominated regions lies on hyperbolas $\xi^{2}=$ const. However, this strategy does not seem to work. Instead, the radial scaling argument on page 35 yields boundaries $s \sim r^{-\gamma}$ with $\gamma<1$, which break Lorentz symmetry. Clearly, all the contributions which are not Lorentz invariant must cancel each other as $\varepsilon \searrow 0$. This seems possible only by working with several layers which have a different scaling in $r$. As a consequence, these layers must also scale differently in $\varepsilon$. In short, we can say that the regularization should involve several length scales.

- As explained in Section 9, the effect of the regularization tails on $\mathcal{M}\left[A_{x y}^{\varepsilon}\right]$ changes drastically near the origin. This seems to imply that as $\varepsilon \searrow 0$, the operator $\mathcal{M}\left[A_{x y}^{\varepsilon}\right]$ will develop singularities at the origin, unless these singularities are compensated by additional regularization tails which are active near $\xi=0$. In other words, the admissible regularizations should involve additional cusplike regions close to the origin. 
- In Section 10 we saw that the behavior of $\mathcal{M}\left[A_{x y}^{\varepsilon}\right]$ near infinity requires special attention. Our method of introducing a shear of the surface states changes the behavior of the surface states considerably if their energy is large. This suggests that the fermionic projector of the vacuum should have a nontrivial high-energy structure.

In any case, these considerations suggest that the fermionic projector of the vacuum should have a complicated structure on the Planck scale. At present, getting more detailed information than what we just discussed seems difficult. Also, in view of the fact that direct measurements on the Planck scale are out reach, it might seem rather speculative to further discuss possible regularizations. The good news coming out of our analysis is that we do not need to know the detailed structure of the fermionic projector on the Planck scale. Since we know that $P^{\varepsilon}$ and $\mathcal{M}\left[A_{x y}^{\varepsilon}\right]$ can be arranged to have a well defined continuum limit, it is consistent to work with $P$ and $\mathcal{M}$ as Lorentz invariant distributions in Minkowski space.

\section{A The Weight Factors $\rho_{\beta}$}

In the ansatz for the fermionic projector of the vacuum (2.11) we introduced weight factors $\rho_{\beta}$. We now discuss the physical meaning of these weight factors and mention their implication for the analysis of the continuum limit.

First of all, we point out that the weights are of no importance for the constructions in this paper. By choosing $\rho_{\beta}=1$, one gets back to the setting of [6]. The motivation for introducing general weights is that this gives more freedom for choosing the vacuum Dirac sea configuration. This additional freedom is of advantage in the stability analysis [11].

At first sight, the weights $\rho_{\beta}$ might seem to contradict physical observations. However, a careful consideration shows that the weights do not lead to obvious contradictions, as we now explain. First, the weights are not in conflict with the proper normalization of the fermionic states (see [6, $\$ 2.6$ and Appendix C]). Namely, thinking of the fermionic projector as being the continuum limit of suitable discretizations, one can arrange Dirac seas with smaller weights for example by occupying the states on the lower mass shell more sparsely. Second, it is not clear how to measure the weights in experiments. For example, one might expect that the weights have an influence on the scattering amplitudes; for instance, it might seem more likely for pair creation to occur if the weights are larger. To see that this argument is incorrect, one has to keep in mind that the scattering amplitudes are computed from the Dirac propagator (and possibly the propagators of the gauge fields), where the weights do not enter. Thus scattering experiments do not seem appropriate for measuring the weights. Indeed, even after discussing the issue with several high energy physicists, the author cannot think of any experiment to measure the weights. It would clearly be desirable to measure the weights $\rho_{\beta}$ or to have a theoretical argument which determines the weights. However, as long as these measurements have not been carried out and no such argument has been given, it seems most honest to treat the weights $\rho_{\beta}$ as free positive parameters.

However, the weights have implications on the analysis of the continuum limit (see $\underline{6}$, Chapter 4]). More precisely, the factors $\rho_{\beta}$ enter the chiral asymmetry matrix $X$, which in [6, §2.3] was introduced by

$$
X=\bigoplus_{a=1}^{N} \bigoplus_{\beta=1}^{3} X_{a \beta},
$$


where the direct summands $X_{a \beta}$ are the identity matrix in the massive sectors and equal the chiral projector $\chi_{L}$ in the neutrino sector. To take into account the weights, we introduce for every Dirac sea a parameter $\rho_{a \beta}>0$ and set

$$
X_{a \beta}=\left\{\begin{array}{cl}
\rho_{a \beta} \mathbb{1} & \text { in the massive sectors } \\
\rho_{a \beta} \chi_{L} & \text { in the neutrino sectors }
\end{array}\right.
$$

When introducing an interaction, we must satisfy the causality compatibility condition (see [6, Def. 2.3.2])

$$
X^{*}(i \not \partial+\mathcal{B}-m Y)=(i \not \partial+\mathcal{B}-m Y) X .
$$

This condition makes it impossible to introduce gauge fields which describe an interaction of Dirac seas with different weights. However, in [6] a similar problem occurred with the neutrino sector, and this problem was overcome by introducing the dynamical mass matrices and by transforming to the effective gauge fields (see [6, Chapters 7 and 8]). Furthermore, the condition (A.1) was weakened in [6, Def. 7.1.1]. In view of these constructions, the weight factors $\rho_{\beta}$ do not lead to obvious problems. However, the implications of the weight factors should be analyzed carefully when working out the continuum limit in more detail.

Acknowledgments: I want to thank Joel Smoller and the referee for helpful comments on the manuscript.

\section{References}

[1] J.C. Baez, "An Introduction to Spin Foam Models of Quantum Gravity and BF Theory," Lect.Notes Phys. 543 (2000) 25-94

[2] L. Bombelli, J. Lee, D. Meyer, R. Sorkin, "Space-time as a causal set," Phys. Rev. Lett. 59 (1987) 521-524

[3] A.H. Chamseddine, A. Connes, "The spectral action principle," Commun. Math. Phys. 186 (1997) 731-750

[4] A. Connes, "Noncommutative Geometry," Academic Press (1994)

[5] A. Diethert, F. Finster, D. Schiefeneder, "Fermion systems in discrete space-time exemplifying the spontaneous generation of a causal structure," arXiv:0710.4420 [mathph] (2007)

[6] F. Finster, "The Principle of the Fermionic Projector," AMS/IP Studies in Advanced Mathematics 35 (2006)

[7] F. Finster, "A variational principle in discrete space-time - existence of minimizers," math-ph/0503069, Calc. Var. and Partial Diff. Eq. 29 (2007) 431-453

[8] F. Finster, "Fermion systems in discrete space-time," hep-th/0601140, J. Phys.: Conf. Ser. 67 (2007) 012048

[9] F. Finster, "Fermion systems in discrete space-time - outer symmetries and spontaneous symmetry breaking," math-ph/0601039, Adv. Theor. Math. Phys. 11 (2007) 91-146 
[10] F. Finster, "From discrete space-time to Minkowski space: Basic mechanisms, methods and perspectives," arXiv:0712.0685 [math-ph] (2007)

[11] F. Finster, S. Hoch, "An action principle for the masses of Dirac particles," arXiv:0712.0678 [math-ph] (2007)

[12] S.W. Hawking, "Space-time Foam," Nucl. Phys. B144 (1978) 349-362

[13] A. Iqbal, N. Nekrasov, A. Okounkov, C. Vafa, "Quantum foam and topological strings," arXiv:hep-th/0312022v2 (2003)

[14] R. Oeckl, "Discrete Gauge Theory: From Lattices to TQFT," Imperial College Press (2005)

[15] D. Oriti, "A quantum field theory of simplicial geometry and the emergence of spacetime," J. Phys.: Conf. Ser. 67 (2007) 012052

[16] T. Regge, R.M. Williams, "Discrete structures in gravity," arXiv:gr-qc/0012035, J.Math.Phys. 41 (2000) 3964-3984

[17] J. A. Wheeler, in "Relativity, Groups and Topology," edited by B.S. and C.M. DeWitt, Gordon and Breach, New York (1964)

[18] C. Rovelli, "Quantum Gravity," Cambridge University Press (2004)

NWF I - Mathematik, Universität Regensburg, 93040 Regensburg, Germany,

Felix.Finster@mathematik.uni-regensburg.de 